\title{
Bifurcação de pontos axiumbílicos e ciclos axiais de superfícies imersas em $\mathbb{R}^{4}$
}

\author{
Flausino Lucas Neves Spindola
}

TESE APRESENTADA

\author{
$\mathrm{AO}$, \\ INSTITUTO DE MATEMÁTICA E ESTATÍSTICA \\ DA
UNIVERSIDADE DE SÃO PAULO \\ OBTENÇÄA DO TÍTULO \\ DE \\ DOUTOR EM CIÊNCIAS
}

Programa de Pós-Graduação em Matemática Aplicada

Orientador: Prof. Dr. Jorge Manuel Sotomayor Tello 


\section{Bifurcação de pontos axiumbílicos e ciclos axiais de superfícies imersas em $\mathbb{R}^{4}$}

Esta versão da tese contém as correções e alterações sugeridas pela Comissão Julgadora durante a defesa da versão original do trabalho, realizada em 06/05/2015. Uma cópia da versão original está disponível no Instituto de Matemática e Estatística da Universidade de São Paulo.

Banca Examinadora:

- Prof. Dr. Jorge Manuel Sotomayor Tello - IME-USP

- Prof. Dr. Antônio Carlos Asperti - IME-USP

- Prof. Dr. Ronaldo Alves Garcia - IME-UFG

- Prof. Dr. Luís Fernando de Osório Melo - IMC-UNIFEI

- Prof. Dr. Paulo Ricardo da Silva - IBILCE-UNESP 


\section{Resumo}

O objetivo deste trabalho é descrever a estrutura das linhas axiais principais e médias

de imersões de superfície $M^{2}$ em $\mathbb{R}^{4}$ na vizinhança de pontos axiumbílicos. Pontos axiumbílicos são aqueles nos quais a elipse de curvatura se degenera em um círculo. Estudamos a perturbação dos ciclos axiais principais, e obtemos resultados sobre a genericidade dos ciclos axiais principais hiperbólicos e semi-hiperbólicos.

Palavras-chave: Linhas Axiais, Axiumbílicos, Ciclos Axiais, Configuração Axial 


\section{Abstract}

The aim of this work is to describe the structure of principal and mean axial lines of immersions of surface $M^{2}$ into $\mathbb{R}^{4}$, close to axiumbilic points. Axiumbilic points are those which the ellipse of curvature denegerate in a circle. We study the perturbation of principal axial cycles, and we obtain results about genericity of hyperbolic and semihyperbolic principal axial cycles.

Keywords: Axial Lines, Axiumbilic Points, Axial Cycles, Axial Configurations 


\section{Agradecimentos}

Há duas pessoas fundamentais na realização deste trabalho, as quais tiveram muita (e muita mesmo!) paciência em me ensinar e acompanhar durante os estudos: Jorge Sotomayor e Ronaldo Garcia. Levarei por toda a vida um enorme sentimento de gratidão pela oportunidade que tive de estudar com estes professores. De fato é uma satisfação enorme participar desse "grupo aguerrido" de equações da geometria. Débora Lopes é uma amiga que deu grande apoio e força. Sempre com muito entusiasmo, realizou encontros proveitosos de nosso grupo de pesquisa na UFS.

Não posso esquecer dos amigos e demais pessoas com as quais convivi no ambiente da Universidade, tão diverso e proveitoso. Vou evitar fazer uma lista de nomes, mas agradeço aqui a todos os colegas e amigos que, de forma direta ou indireta, estiveram comigo em algum momento. Todos foram muito importantes pra mim!

Por fim, agradeço a minha mãe, Ana, e a minhas irmãs, Ana Carolina e Valéria, pelo constante empenho e preocupação com relação a meus estudos de doutorado. Do ponto de vista pessoal, este texto simboliza a conclusão de um processo de formação que se iniciou há mais de 20 anos, na pré-escola. 


\section{Sumário}

Lista de Figuras $\quad$ ix

1 Configurações Axiais de Superfícies Imersas em $\mathbb{R}^{4} \quad 1$

1.1 Equação Diferencial das Linhas Axiais . . . . . . . . . . . . . . . . . 1

1.2 Conceituação de Configurações Axiais . . . . . . . . . . . . . . . . 8

1.3 Equação Diferencial em uma Carta de Monge . . . . . . . . . . . . . . . 11

2 Pontos Axiumbílicos Estáveis 17

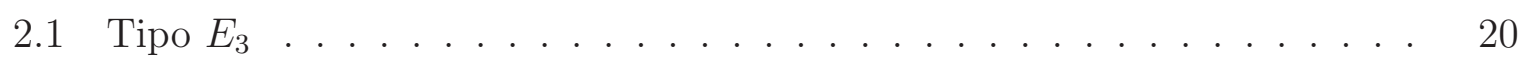

2.2 Tipo $E_{4} \ldots \ldots \ldots \ldots \ldots \ldots$

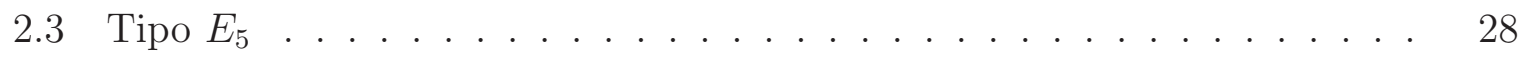

2.4 Diagrama dos Pontos Estáveis . . . . . . . . . . . . . . . . 30

3 Pontos Axiumbílicos de Codimensão 1

3.1 O Ponto Axiumbílico $E_{34}^{1} \ldots \ldots \ldots$. . . . . . . . . . . . . 33

3.2 O ponto Axiumbílico $E_{45}^{1} \ldots \ldots \ldots \ldots \ldots \ldots$

3.3 Transversalidade e Estratificação . . . . . . . . . . . . . . . . 55

4 Ciclos Axiais Principais e Genericidade $\quad 57$

4.1 Primeira e Segunda Derivadas da Aplicação de Poincaré . . . . . . . . . . . . . . . . . . . . . . . . 59

4.2 Genericidade de Ciclos Hiperbólicos . . . . . . . . . . . . . . . . . . . 62

4.3 Genericidade de Ciclos Semi-Hiperbólicos . . . . . . . . . . . . . . 67

4.4 Sobre a Condição $\tau_{g, 2} \neq 0 \ldots \ldots \ldots$. . . . . . . . . . . 69

4.5 Superfícies em torno de Ciclos Axiais Principais . . . . . . . . . . . . . . 72

$\begin{array}{ll}\text { A Teorema de Transversalidade de Thom } & 79\end{array}$

Referências Bibliográficas . . . . . . . . . . . . . . . . 84

Referências Bibliográficas $\quad 85$ 


\section{Lista de Figuras}

1.1 Aplicação Curvatura Normal e Elipse de Curvatura $\varepsilon_{\alpha}(\mathfrak{p})$. . . . . . . . . 4

1.2 Elipse de Curvatura $\varepsilon_{\alpha}(\mathfrak{p})$ ilustrando Pontos Extremais. . . . . . . . . . . 5

1.3 Transversalidade e Contato Quadrático das Curvas $a_{0}=0$ e $a_{1}=0$ em p. $\quad 8$

1.4 Ilustração da projeção em $M$ das linhas $\mathcal{L}_{\alpha}$ do campo de Lie-Cartan na vizinhança de um ponto $\mathfrak{p} \in\left(M \mathcal{U}_{\alpha}\right)$. Os números ilustram a projeção de cada linha, temos portanto as cruzes determinadas por $1-3$ e $2-4$. . 10

1.5 Ilustração da rotação realizada no Lema 1.11, considerando $T \neq 0$. . . . 15

2.1 Gráfico de $R(p)$ no caso $E_{3}$. . . . . . . . . . . . . . . 20

2.2 Curvas integrais de $\left.X\right|_{\mathcal{G}=0}$ na vizinhança do eixo projetivo no caso $E_{3} \ldots \quad 21$

2.3 Configuração Axial Principal e Configuração Axial Média ao redor de um

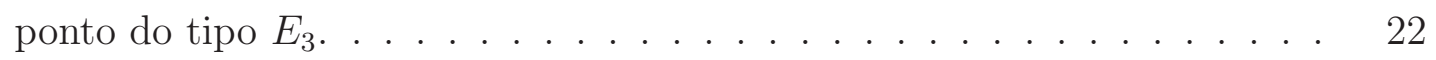

2.4 Gráfico de $R(p)$ no caso $E_{4}$, caso $(I . i) \ldots \ldots \ldots \ldots \ldots$

2.5 Gráfico de $R(p)$ no caso $E_{4}$, caso $(I . i i) \ldots \ldots \ldots \ldots$

2.6 Gráfico de $R(p)$ no caso $E_{4}$, caso $($ I.iii $) \ldots \ldots \ldots \ldots \ldots$. . . . . . 24

2.7 Gráfico de $R(p)$ no caso $E_{4}$, caso $(I . i v) \ldots \ldots \ldots \ldots \ldots$

2.8 Gráfico de $R(p)$ no caso $E_{4}$, caso $(I I) \ldots \ldots \ldots \ldots$

2.9 Curvas integrais de $\left.X\right|_{\mathcal{G}}=0$ na vizinhança do eixo projetivo no caso $E_{4}$. 27

2.10 Configuração Axial Principal e Configuração Axial Média ao redor de um ponto do tipo $E_{4} \ldots \ldots \ldots \ldots$. . . . . . . . . . . . . . . 27

2.11 Gráfico de $R(p)$ no caso $E_{5} \ldots \ldots \ldots \ldots \ldots$

2.12 Curvas integrais de $\left.X\right|_{\mathcal{G}}=0$ na vizinhança do eixo projetivo no caso $E_{5} . \quad 29$

2.13 Configuração Axial Principal e Configuração Axial Média ao redor de um ponto do tipo $E_{5} \ldots \ldots \ldots \ldots \ldots$. . . . . . . . . . . . . . . . . 29

2.14 Diagrama dos Pontos Axiumbílicos Estáveis. . . . . . . . . . . . . 30

3.1 Configuração Axial Principal e Configuração Axial Média na vizinhança de um ponto do tipo $E_{34}^{1} \ldots \ldots \ldots \ldots$. . . . . . . . . . . . . . . . . . . . . . . 35

3.2 Seis Componentes onde ocorre ponto do tipo $E_{34}^{1} \ldots \ldots \ldots$

3.3 Gráfico de $R$ no caso $A$. . . . . . . . . . . . . . . . . . . . 36

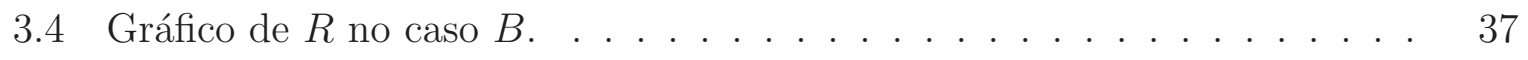

3.5 Gráfico de $R$ no caso $C \ldots \ldots \ldots \ldots$. . . . . . . . . . . . 37 
3.6 Gráfico de $R$ no caso $D . \ldots \ldots \ldots$

3.7 Gráfico de $R$ no caso E. . . . . . . . . . . . . . . . . . . . . . . . 40

3.8 Gráfico de $R$ no caso $F$. . . . . . . . . . . . . . . . . . 40

3.9 Curvas integrais de $\left.X\right|_{\mathcal{G}}=0$ na vizinhança do eixo projetivo no caso $E_{34}^{1} . \quad 42$

3.10 Configuração Axial Principal e Configuração Axial Média na vizinhança de um ponto do tipo $E_{34}^{1} \ldots \ldots$. . . . . . . . . . . . . . . . 42

3.11 Configurações Axiais na vizinhança de pontos axiumbílicos. $E_{3}$ (esquerda), $E_{34}^{1}$ (centro) and $E_{4}$ (direita). . . . . . . . . . . . . . . 44

3.12 Diagrama de Bifurcação das Configurações Axiais próximas de um ponto axiumbílico $E_{34}^{1}$ e a estrutura de separatrizes. . . . . . . . . . . . 45

3.13 As 4 singularidades $p_{i}$. . . . . . . . . . . . . . . . . . . 49

3.14 Folhas da superfície de Lie-Cartan. O eixo $p$ está inclinado para favorecer a visualização. . . . . . . . . . . . . . . . . . . . . . . . . . 50

3.15 Ilustração da superfície com os pontos críticos tipo Morse e retrato de fase do campo de Lie-Cartan. . . . . . . . . . . . . . . . . . . . . 51

3.16 Configurações Axiais na vizinhança de um ponto axiumbílico do tipo $E_{4,5}^{1}$. 51

3.17 Campo de Lie-Cartan próximo de um ponto axiumbílico $E_{45}^{1}$ e a configuração axial (principal e média) . . . . . . . . . . . . . 52

3.18 Ponto Axiumbílico $E_{45}^{1}$. Os pontos $E_{4}$ and $E_{5}$ colidem em um ponto tipo $E_{45}^{1}$, e após são eliminados e não há pontos axiumbílicos na vizinhança. . 53

3.19 Diagrama de Bifurcação da configuração axial próximo do ponto axiumbílico $E_{45}^{1}$ e a estrutura de separatrizes. . . . . . . . . . . . . 54

3.20 Superfície de Lie-Cartan. A esquerda, com dois pontos axiumbílicos, ao centro com quatro pontos singulares, e à direita os quatro níveis regulares.

4.1 Ilustração da Elipse de Curvatura sobre o ciclo axial segundo a parametrização da Proposição $4.3 \ldots$. . . . . . . . . . . . . . . . . . . 58

4.2 Parametrização local em torno do ciclo axial principal, ilustrando a aplicação de primeiro retorno. . . . . . . . . . . . . . . . . . 59

4.3 Ilustração, à esquerda, do caso em que $F^{-1}(0,0,0)=\emptyset$, e à direita e do caso $F^{-1}(0,0,0) \neq \emptyset \ldots \ldots \ldots \ldots \ldots \ldots \ldots \ldots \ldots \ldots \ldots \ldots \ldots \ldots$ 


\section{Introdução}

Nesta tese apresentamos um estudo das linhas axiais de superfícies imersas em $\mathbb{R}^{4}$, bem como análise da alteração estrutural destas linhas por pequenas perturbações no espaço das imersões de superfícies em $\mathbb{R}^{4}$.

Iniciamos este trabalho definindo estas linhas, a partir dos eixos da elipse de curvatura (daí o nome Axial, ver Seção 1.1). Esta elipse é a imagem no plano normal à superfície da aplicação segunda forma fundamental restrita ao círculo unitário tangente à superfície (Figura 1.1). As linhas correspondentes ao eixo maior da elipse são chamadas linhas axiais principais e as linhas correspondentes ao eixo menor da elipse são chamadas linhas axiais médias (Figura 1.2, Figura 1.4). Damos uma conceituação precisa destas linhas, e das Configurações Axiais Principais e Médias na Seção 1.2.

Existem pontos na superfície em que a elipse de curvatura se degenera em um círculo. Os pontos onde tal situação ocorre são chamados umbílicos axiais ou axiumbílicos. No capítulo 2 efetuamos a descrição das configurações axiais (principal e média) na vizinhança de três tipos de pontos axiumbílicos, denotados por $E_{3}, E_{4}$ e $E_{5}$ (Figura 2.3, Figura 2.10, Figura 2.13). A letra $E$ se deve a notação de elipse e o índice se refere ao número de setores hiperbólicos envolvidos na descrição destes pontos. Estes pontos são "estáveis" por pequenas perturbações no espaço das imersões (Proposição 2.4), em analogia com os pontos Darbouxianos no caso de superfícies imersas em $\mathbb{R}^{3}$ ( [42], pg. 45).

No contexto de Teoria das Bifurcações, estudamos as configurações de transição entre os pontos descritos no capítulo 2. No terceiro capítulo explicamos o comportamento das linhas axiais na vizinhança de pontos axiumbílicos que denotamos por $E_{34}^{1}$ e $E_{45}^{1}$ (Figura 3.10, Figura 3.16). O número 1 sobrescrito denota que estes pontos são de codimensão 1 , ou seja, descritos por meio de variação a um parâmetro, ou uma curva suave no espaço das imersões $\mathcal{C}^{r}$ de uma superfície $M$ em $\mathbb{R}^{4}$. O ponto $E_{34}^{1}$ trata da transição entre um ponto axiumbílico do tipo $E_{3}$ e um do tipo $E_{4}$. O ponto $E_{45}^{1}$ se caracteriza pela "colisão" entre um ponto do tipo $E_{4}$ e um do tipo $E_{5}$, e após a "colisão" a consequente eliminação de pontos axiumbílicos numa vizinhança. Nos dois casos, efetuamos variação a um parâmetro no espaço das imersões, numa analogia ao estudo das bifurcações do tipo sela-nó no caso das equações diferenciais no plano [39].

No capítulo 4 tratamos dos ciclos axiais principais, os quais são linhas periódicas de 
curvatura axial principal. Estudamos a Transformação de Primeiro Retorno dos Ciclos Axiais, também conhecida como Transformação de Poincaré (denotada por $\pi$ ), e exprimimos as Fórmulas da Primeira e Segunda Derivadas da Transformação de Poincaré em termos de funções geométricas definidas na vizinhança deste ciclo (Proposição 4.8, Proposição 4.10). De posse destas fórmulas, analisamos a hiperbolicidade $\left(\pi^{\prime}(0) \neq 1\right)$ e semi-hiperbolicidade $\left(\pi^{\prime}(0)=1\right.$ e $\left.\pi^{\prime \prime}(0) \neq 0\right)$ destes ciclos. Como conclusão, duas proposições são demonstradas:

1) Dada uma imersão com um ciclo axial principal não-hiperbólico, é possível encontrar imersão suficientemente próxima que torna tal ciclo hiperbólico (Teorema 4.9).

2) Dada uma imersão com um ciclo axial principal o qual não é hiperbólico e nem semihiperbólico, é possível encontrar imersão suficientemente próxima que torna tal ciclo semi-hiperbólico (Teorema 4.11). 


\section{Capítulo 1}

\section{Configurações Axiais de Superfícies Imersas em $\mathbb{R}^{4}$}

\subsection{Equação Diferencial das Linhas Axiais}

Seja $\alpha: M^{2} \longrightarrow \mathbb{R}^{4}$ uma imersão $\mathcal{C}^{r}, r \geq 4$, de uma superfície suave orientada no espaço $\mathbb{R}^{4}$, este último com uma orientação fixada e dotado do produto interno euclideano. Assuma que $(x, y)$ seja uma carta positiva e que $\left\{\alpha_{x}, \alpha_{y}, N_{1}, N_{2}\right\}$ seja um referencial positivo onde, para cada $\mathfrak{p} \in M,\left\{\alpha_{x}, \alpha_{y}\right\}_{\mathfrak{p}}$ é base do plano tangente $T_{\mathfrak{p}} M$ e $\left\{N_{1}, N_{2}\right\}_{\mathfrak{p}}$ é base do plano normal $N_{\mathfrak{p}} M$.

Na carta $(x, y)$, podemos escrever a primeira e segunda formas fundamentais da superfície $M$, utilizando a estrutura geométrica do espaço euclideano, a saber:

$$
\begin{aligned}
I_{\alpha} & :=<D \alpha, D \alpha> \\
& =<\alpha_{x} d x+\alpha_{y} d y, \alpha_{x} d x+\alpha_{y} d y> \\
& =E d x^{2}+2 F d x d y+G d y^{2}
\end{aligned}
$$

sendo $E=\left\langle\alpha_{x}, \alpha_{x}\right\rangle, F=\left\langle\alpha_{x}, \alpha_{y}\right\rangle$ e $G=\left\langle\alpha_{y}, \alpha_{y}\right\rangle$. Quanto à segunda forma fundamental, esta é escrita na forma:

$$
I I_{\alpha}=I I_{\alpha}^{1} N_{1}+I I_{\alpha}^{2} N_{2}
$$

onde cada $I I_{\alpha}^{i}, i=1,2$ é descrito por:

$$
\begin{aligned}
I I_{\alpha}^{i} & :=<D^{2} \alpha, N_{i}> \\
& =<\alpha_{x x} d x^{2}+2 \alpha_{x y} d x d y+\alpha_{y y} d y^{2}, N_{i}> \\
& =e_{i} d x^{2}+2 f_{i} d x d y+g_{i} d y^{2},
\end{aligned}
$$

sendo $e_{i}=\left\langle\alpha_{x x}, N_{i}\right\rangle, f_{i}=\left\langle\alpha_{x y}, N_{i}\right\rangle$ e $g_{i}=\left\langle\alpha_{y y}, N_{i}\right\rangle$. 
Para cada $\mathfrak{p} \in M$, definimos o vetor curvatura média $H(p)$ por:

$$
H=h_{1} N_{1}+h_{2} N_{2}
$$

sendo

$$
h_{i}=\frac{E g_{i}-2 F f_{i}+G e_{i}}{2\left(E G-F^{2}\right)}
$$

e, tomando um vetor não-nulo $v$ em $T_{\mathfrak{p}} M$, definimos o vetor curvatura normal por:

$$
k_{n}=k_{n}(\mathfrak{p}, v):=\frac{I I_{\alpha}(v)}{I_{\alpha}(v)}=\frac{I I_{\alpha}^{1}(v)}{I_{\alpha}(v)} N_{1}+\frac{I I_{\alpha}^{2}(v)}{I_{\alpha}(v)} N_{2} .
$$

Note que, sendo $v$ vetor não-nulo e $\lambda \neq 0$,

$$
\begin{aligned}
k_{n}(\mathfrak{p}, \lambda v) & =\frac{I I_{\alpha}(\lambda v)}{I_{\alpha}(\lambda v)} \\
& =\frac{\lambda^{2} \cdot I I_{\alpha}(v)}{\lambda^{2} \cdot I_{\alpha}(v)} \\
& =\frac{I I_{\alpha}(v)}{I_{\alpha}(v)} \\
& =k_{n}(\mathfrak{p}, v) .
\end{aligned}
$$

Isto nos permite falar em vetor curvatura normal na direção de $v$, e portanto podemos considerar $k_{n}$ restrito ao círculo unitário $S_{\mathfrak{p}}^{1}:=\left\{v \in T_{\mathfrak{p}} M: I_{\mathfrak{p}}(v)=1\right\}$. Para interpretar geometricamente o vetor curvatura normal, considere $v \in S_{\mathfrak{p}}^{1}$. Podemos então tomar uma curva parametrizada pelo comprimento de arco $\gamma:(-\varepsilon, \varepsilon) \longrightarrow M$ satisfazendo $\gamma(0)=\mathfrak{p}$ e $\gamma^{\prime}(0)=v$. O vetor curvatura normal $k_{n}(\mathfrak{p}, v)$ é a projeção no plano normal $N_{\mathfrak{p}} M$ do vetor aceleração $\gamma^{\prime \prime}(0)$. De fato:

$$
\begin{aligned}
k_{n}(\mathfrak{p}, v) & =I I_{\alpha}^{1}\left(\gamma^{\prime}(0)\right) N_{1}+I I_{\alpha}^{2}\left(\gamma^{\prime}(0)\right) N_{2} \\
& =\left\langle\gamma^{\prime \prime}(0), N_{1}>\cdot N_{1}+\left\langle\gamma^{\prime \prime}(0), N_{2}>\cdot N_{2}\right.\right.
\end{aligned}
$$

É importante ressaltar que esta intepretação independe da escolha da curva $\gamma \operatorname{com} \gamma^{\prime}(0)=$ $v$. Para uma melhor compreensão desta aplicação, considere um sistema de coordenadas ortonormal em $\mathfrak{p}$, isto é, com $E=G=1$ e $F=0$ no ponto $\mathfrak{p}$. Então, podemos escrever $v=\cos t \cdot \alpha_{x}+\sin t \cdot \alpha_{y}$. Neste caso,

$$
\begin{aligned}
k_{n}(\mathfrak{p}, v) & =\left(e_{1} \cdot \cos ^{2} t+2 \cdot f_{1} \cdot \cos t \cdot \sin t+g_{1} \cdot \sin ^{2} t\right) N_{1} \\
& +\left(e_{2} \cdot \cos ^{2} t+2 \cdot f_{2} \cdot \cos t \cdot \sin t+g_{2} \cdot \sin ^{2} t\right) N_{2}
\end{aligned}
$$


e

$$
H(\mathfrak{p})=\frac{e_{1}+g_{1}}{2} \cdot N_{1}+\frac{e_{2}+g_{2}}{2} \cdot N_{2}
$$

De acordo com [30], [31], utilizando as identidades trigonométricas $\cos 2 t=\cos ^{2} t-\sin ^{2} t$ e $\sin 2 t=2 \sin t \cos t$, temos que

$$
\begin{aligned}
k_{n}(\mathfrak{p}, v) & =\left(\frac{e_{1}-g_{1}}{2} \cdot \cos 2 t+f_{1} \cdot \sin 2 t\right) \cdot N_{1} \\
& +\left(\frac{e_{2}-g_{2}}{2} \cdot \cos 2 t+f_{2} \cdot \sin 2 t\right) \cdot N_{2} \\
& +H(p) .
\end{aligned}
$$

Em notação matricial:

$$
k_{n}(\mathfrak{p}, v)-H(\mathfrak{p})=\left[\begin{array}{cc}
\frac{e_{1}-g_{1}}{2} & f_{1} \\
\frac{e_{2}-g_{2}}{2} & f_{2}
\end{array}\right] \cdot\left[\begin{array}{c}
\cos 2 t \\
\sin 2 t
\end{array}\right]
$$

Portanto, a aplicação $k_{n}$ restrita ao círculo unitário é uma aplicação afim, e sua imagem descreve em $N_{\mathfrak{p}} M$ uma elipse centrada em $H(\mathfrak{p})$, a qual chamamos de elipse de curvatura de $\alpha$ no ponto $\mathfrak{p}$, denotada por $\varepsilon_{\alpha}(\mathfrak{p})$. A área desta elipse é dada por:

$$
\begin{aligned}
A\left(\varepsilon_{\alpha}(\mathfrak{p})\right) & =A\left(S_{\mathfrak{p}}^{1}\right) \cdot\left|\begin{array}{ll}
\frac{e_{1}-g_{1}}{2} & f_{1} \\
\frac{e_{2}-g_{2}}{2} & f_{2}
\end{array}\right| \\
& =\pi \cdot \frac{\left(e_{1}-g_{1}\right) f_{2}-\left(e_{2}-g_{2}\right) f_{1}}{2} .
\end{aligned}
$$

Quando $\left(e_{1}-g_{1}\right) f_{2}-\left(e_{2}-g_{2}\right) f_{1}=0$, a elipse se degenera em um segmento de reta ou um ponto. Sendo ainda $\left(e_{1}-g_{1}\right) f_{2}-\left(e_{2}-g_{2}\right) f_{1} \neq 0$, temos o caso especial em que $\varepsilon_{\alpha}(\mathfrak{p})$ se degenera em um círculo, que será melhor discutido a seguir. Além disso, restrita a $S_{\mathfrak{p}}^{1}$, temos que $k_{n}=I I_{\alpha}$, por ser uma aplicação quadrática, recobre duplamente a elipse de curvatura.

Observação 1.1. A equação (1.3) pode ser vista como:

$$
T(t)=\left[\begin{array}{ll}
\frac{e_{1}-g_{1}}{2} & f_{1} \\
\frac{e_{2}-g_{2}}{2} & f_{2}
\end{array}\right] \cdot\left[\begin{array}{c}
\cos 2 t \\
\sin 2 t
\end{array}\right]
$$

onde $t$ é o ângulo medido no sentido anti-horário entre $\alpha_{x}$ e o vetor $v$. Utilizando as relações trigonométricas $\cos (\theta+\varphi)=\cos (\theta) \cos (\varphi)-\sin (\theta) \sin (\varphi)$ e $\sin (\theta+\varphi)=$ $\sin (\theta) \cos (\varphi)+\sin (\varphi) \cos (\theta)$, obtemos:

a) $T(t)=T(t+k \pi), k \in \mathbb{Z}$. Portanto, a pré-imagem de cada ponto de $\varepsilon_{\alpha}(\mathfrak{p})$ é constituída de dois pontos diametralmente opostos em $S_{\mathfrak{p}}^{1}$, e portanto, a cada ponto de $\varepsilon_{\alpha}(\mathfrak{p})$ está 
4 CAPÍTULO 1. CONFIGURAÇÕES AXIAIS DE SUPERFÍCIES IMERSAS EM $\mathbb{R}^{4}$ associada uma direção em $T_{\mathfrak{p}} M$.

b) $T\left(t+\frac{\pi}{2}\right)=-T(t)$. Logo, a dois pontos da elipse de curvatura simétricos com relação a $H(\mathfrak{p})$ estão associadas duas direções ortogonais em $T_{\mathfrak{p}} M$, formando assim uma cruz em $T_{\mathfrak{p}} M$.

c) A elipse de curvatura, por ser imagem de um círculo em $T_{\mathfrak{p}} M$, é invariante por rotações no plano tangente e no plano normal. Logo, o seu centro $H(\mathfrak{p})$, assim como sua área são invariantes da imersão $\alpha$.

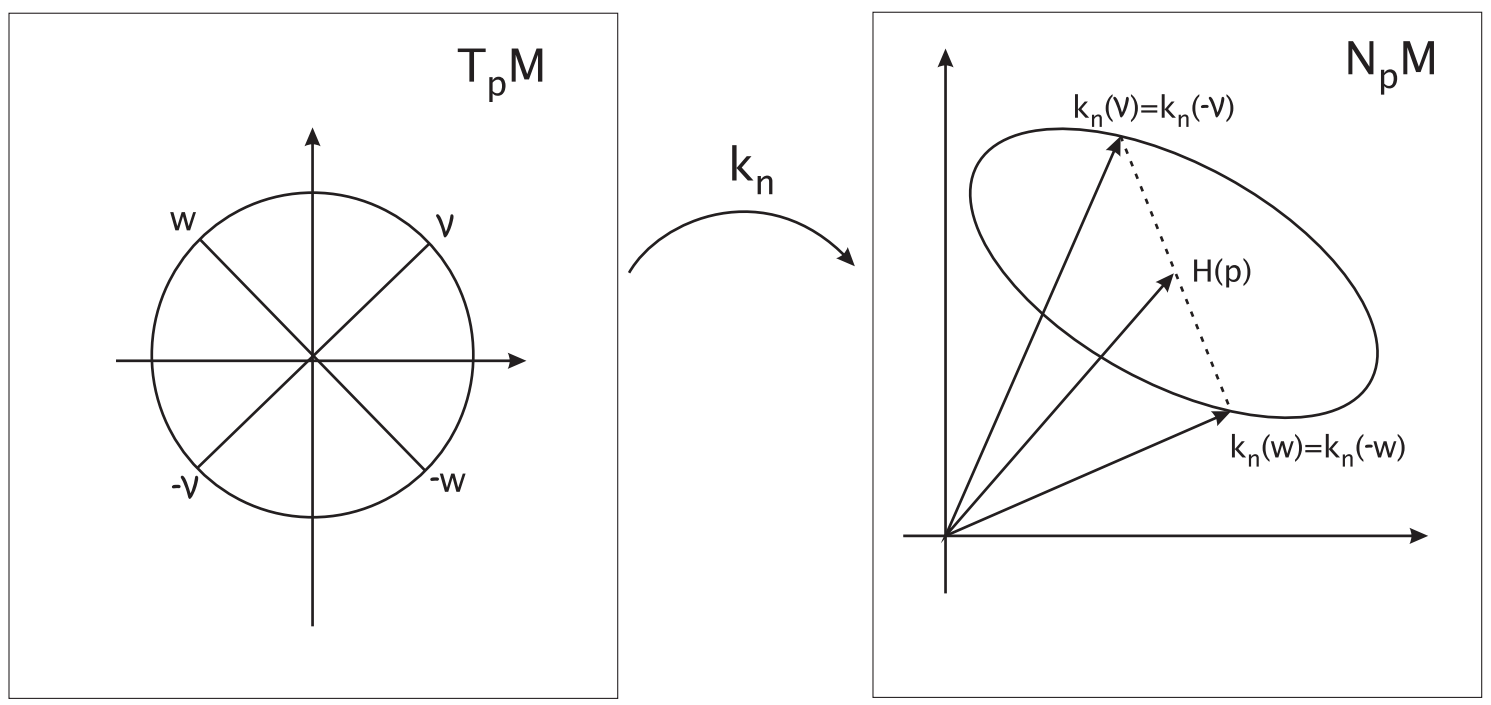

Figura 1.1: Aplicação Curvatura Normal e Elipse de Curvatura $\varepsilon_{\alpha}(\mathfrak{p})$.

Considere a função

$$
\begin{aligned}
\left\|k_{n}-H\right\|^{2} & :=\left[\frac{e_{1} d x^{2}+2 f_{1} d x d y+g_{1} d y^{2}}{E d x^{2}+2 F d x d y+G d y^{2}}-\frac{E g_{1}-2 F f_{1}+G e_{1}}{2\left(E G-F^{2}\right)}\right]^{2} \\
& +\left[\frac{e_{2} d x^{2}+2 f_{2} d x d y+g_{2} d y^{2}}{E d x^{2}+2 F d x d y+G d y^{2}}-\frac{E g_{2}-2 F f_{2}+G e_{2}}{2\left(E G-F^{2}\right)}\right]^{2}
\end{aligned}
$$

Para cada $\mathfrak{p} \in M$ em que $\varepsilon_{\alpha}(\mathfrak{p})$ não se degenera em um ponto ou círculo, os pontos de máximo e de mínimo desta função determinam quatro pontos sobre a elipse de curvatura $\varepsilon_{\alpha}(\mathfrak{p})$, a saber, aqueles onde a elipse cruza com seus eixos maior e menor.

Conforme ilustramos na Figura 1.2, ao eixo menor $A B$ está associada a cruz $A^{\prime} A^{\prime \prime} B^{\prime} B^{\prime \prime}$ e ao eixo maior $C D$ está associada a cruz $C^{\prime} C^{\prime \prime} D^{\prime} D^{\prime \prime}$. Portanto, para cada $\mathfrak{p} \in M$ em que a elipse é não-degenerada, ficam bem definidas em $T_{\mathfrak{p}} M$ duas cruzes, onde uma cruz está associada ao eixo maior e outra ao eixo menor da elipse de curvatura. Temos assim um Campo de 2-Cruzes em M, o qual chamaremos de Campo de Direções de Curvatura Axial, devido ao sentido geométrico destas cruzes estarem relacionadas com os eixos maior e menor da elipse $\varepsilon_{\alpha}(\mathfrak{p})$. 


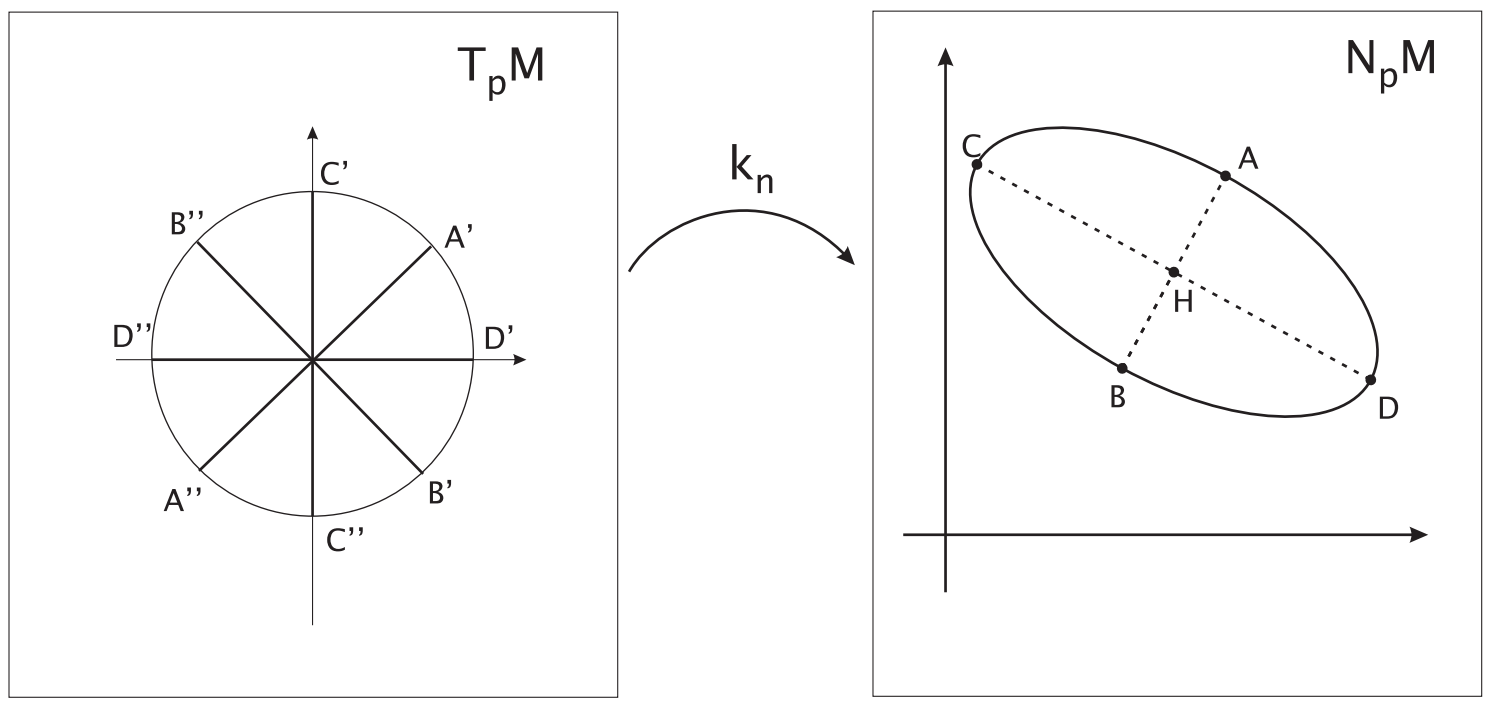

Figura 1.2: Elipse de Curvatura $\varepsilon_{\alpha}(\mathfrak{p})$ ilustrando Pontos Extremais.

Observação 1.2. As cruzes $A^{\prime} A^{\prime \prime} B^{\prime} B^{\prime \prime}$ e $C^{\prime} C^{\prime \prime} D^{\prime} D^{\prime \prime}$ formam entre si ângulo de $\frac{\pi}{4}$. Isto segue da análise feita na Observação 1.1.

Nos pontos $\mathfrak{p} \in M$ em que a elipse se degenera em um círculo, temos que a função $\left\|k_{n}-H\right\|^{2}$ é constante, e portanto o campo de direções de curvatura axial não fica bem definido. Em analogia com o que ocorre com linhas de curvatura de superfícies em $\mathbb{R}^{3}$, chamaremos estes de pontos umbílicos axiais ou pontos axiumbílicos da imersão $\alpha$, e denotaremos o conjunto dos pontos axiumbílicos da imersão $\alpha$ por $\mathcal{U}_{\alpha} \cdot($ [44], [42], [31]).

O problema de determinar as direções de curvatura axial consiste em resolver a equação

$$
J a c\left(\left\|k_{n}-H\right\|^{2}, I_{\alpha}\right)=0
$$

a qual possui quatro soluções para cada $\mathfrak{p}$ fora de $\mathcal{U}_{\alpha}$, e fica indeterminada nos pontos $\mathfrak{p} \in \mathcal{U}_{\alpha}$.

Efetuando os cálculos pertinentes, temos, de acordo com [44] e [42], que a Equação Diferencial das Linhas Axiais é dada por:

$$
a_{4} d y^{4}+a_{3} d y^{3} d x+a_{2} d y^{2} d x^{2}+a_{1} d y d x^{3}+a_{0} d x^{4}=0
$$


6 CAPÍTULO 1. CONFIGURAÇÕES AXIAIS DE SUPERFÍCIES IMERSAS EM $\mathbb{R}^{4}$

onde

$$
\begin{array}{rl}
a_{4} & =-4 F\left(E G-2 F^{2}\right)\left(g_{1}^{2}+g_{2}^{2}\right)+4 G\left(E G-4 F^{2}\right)\left(f_{1} g_{1}+f_{2} g_{2}\right) \\
& +8 F G^{2}\left(f_{1}^{2}+f_{2}^{2}\right)+4 F G^{2}\left(e_{1} g_{1}+e_{2} g_{2}\right)-4 G^{3}\left(e_{1} f_{1}+e_{2} f_{2}\right), \\
a_{3} & =-4 E\left(E G-4 F^{2}\right)\left(g_{1}^{2}+g_{2}^{2}\right)-32 E F G\left(f_{1} g_{1}+f_{2} g_{2}\right) \\
& +16 E G^{2}\left(f_{1}^{2}+f_{2}^{2}\right)-4 G^{3}\left(e_{1}^{2}+e_{2}^{2}\right)+8 E G^{2}\left(e_{1} g_{1}+e_{2} g_{2}\right), \\
& \\
a_{2} & =-12 F G^{2}\left(e_{1}^{2}+e_{2}^{2}\right)+12 E^{2} F\left(E G-4 F^{2}\right)\left(g_{1}^{2}+g_{2}^{2}\right) \\
& +24 E G^{2}\left(e_{1} f_{1}+e_{2} f_{2}\right)-24 E^{2} G\left(f_{1} g_{1}+f_{2} g_{2}\right), \\
& =4 E^{3}\left(g_{1}^{2}+g_{2}^{2}\right)+4 G\left(E G-4 F^{2}\right)\left(e_{1}^{2}+e_{2}^{2}\right) \\
a_{1} & 32 E F G\left(e_{1} f_{1}+e_{2} f_{2}\right)-16 E^{2} G\left(f_{1}^{2}+f_{2}^{2}\right)-8 E^{2} G\left(e_{1} g_{1}+e_{2} g_{2}\right), \\
& \\
a_{0} & =4 F\left(E G-2 F^{2}\right)\left(e_{1}^{2}+e_{2}^{2}\right)-4 E\left(E G-4 F^{2}\right)\left(e_{1} f_{1}+e_{2} f_{2}\right) \\
& +-8 E^{2} F\left(f_{1}^{2}+f_{2}^{2}\right)-4 E^{2} F\left(e_{1} g_{1}+e_{2} g_{2}\right)+4 E^{3}\left(f_{1} g_{1}+f_{2} g_{2}\right) .
\end{array}
$$

Combinando e simplificando as expressões acima para $a_{i}, i=0, \ldots, 4$, temos o seguinte lema:

Lema 1.3 ( [44], [42] pg. 121). Nas equações acima, temos as seguintes relações:

i) $E a_{2}=-6 a_{0}+3 F a_{1}$,

ii) $E^{2} a_{3}=\left(4 F^{2}-E G\right) a_{1}-8 F G a_{0}$,

iii) $E^{3} a_{4}=G\left(E G-4 F^{2}\right) a_{0}+F\left(2 F^{2}-E G\right) a_{1}$.

A partir destas simplificações, podemos enunciar a proposição:

Proposição 1.4 ( [44], [42]). Seja $\alpha: M \longrightarrow \mathbb{R}^{4}$ uma imersão $\mathcal{C}^{r}, r \geq 4$, de uma superfície suave orientada. Denote a primeira forma fundamental por

$$
I_{\alpha}=E d x^{2}+2 F d x d y+G d y^{2}
$$

e a segunda forma fundamental por

$$
I I_{\alpha}=\left(e_{1} d x^{2}+2 f_{1} d x d y+g_{1} d y^{2}\right) N_{1}+\left(e_{2} d x^{2}+2 f_{2} d x d y+g_{2} d y^{2}\right) N_{2},
$$

onde $\left\{N_{1}, N_{2}\right\}$ é um referencial ortonormal, tal que $\left\{\alpha_{x}, \alpha_{y}, N_{1}, N_{2}\right\}$ seja um referencial positivo. 
i) A equação diferencial das linhas axiais é dada por:

$$
\begin{aligned}
\mathcal{G} & =\left[a_{0} G\left(E G-4 F^{2}\right)+a_{1} F\left(2 F^{2}-E G\right)\right] d y^{4} \\
& +\left[-8 a_{0} E F G+a_{1} E\left(4 F^{2}-E G\right)\right] d y^{3} d x \\
& +\left[-6 a_{0} G E^{2}+3 a_{1} F E^{2}\right] d y^{2} d x^{2} \\
& +a_{1} E^{3} d y d x^{3} \\
& +a_{0} E^{3} d x^{4} \\
& =0
\end{aligned}
$$

onde

$$
\begin{aligned}
a_{1} & =4 G\left(E G-4 F^{2}\right)\left(e_{1}^{2}+e_{2}^{2}\right) \\
& +32 E F G\left(e_{1} f_{1}+e_{2} f_{2}\right) \\
& +4 E^{3}\left(g_{1}^{2}+g_{2}^{2}\right) \\
& -8 E^{2} G\left(e_{1} g_{1}+e_{2} g_{2}\right) \\
& -16 E^{2} G\left(f_{1}^{2}+f_{2}^{2}\right),
\end{aligned}
$$

$e$

$$
\begin{aligned}
a_{0} & =4 F\left(E G-2 F^{2}\right)\left(e_{1}^{2}+e_{2}^{2}\right) \\
& -4 E\left(E G-4 F^{2}\right)\left(e_{1} f_{1}+e_{2} f_{2}\right) \\
& +4 E^{3}\left(f_{1} g_{1}+f_{2} g_{2}\right) \\
& -4 E^{2} F\left(e_{1} g_{1}+e_{2} g_{2}\right) \\
& -8 E^{2} F\left(f_{1}^{2}+f_{2}^{2}\right) .
\end{aligned}
$$

ii) Os pontos axiumbilicos de $\alpha$ são caracterizados por $a_{0}=a_{1}=0$.

Conforme o item $(i i)$ da Proposição 1.4, os pontos axiumbílicos são determinados pelas intersecções das curvas $a_{0}(x, y)=0$ e $a_{1}(x, y)=0$. Admitindo, sem perda de generalidade, que elas se intersectam em $(x, y)=(0,0)$, trataremos neste projeto do caso em que tais curvas se intersectam transversalmente em $(0,0)$ e do caso em que elas se tangenciam em $(0,0)$ com contato quadrático, conforme ilustramos na Figura 1.3.

Diremos que o ponto axiumbílico expresso por $(x, y)=(0,0)$ é transversal se

$$
\operatorname{det}\left(\left.\frac{\partial\left(a_{0}, a_{1}\right)}{\partial(x, y)}\right|_{(0,0)}\right)=\left|\begin{array}{ll}
\frac{\partial a_{0}}{\partial x}(0,0) & \frac{\partial a_{0}}{\partial y}(0,0) \\
\frac{\partial a_{1}}{\partial x}(0,0) & \frac{\partial a_{1}}{\partial x}(0,0)
\end{array}\right| \neq 0 .
$$

O ponto axiumbílico expresso por $(x, y)=(0,0)$ será dito de contato quadrático se a 
matriz:

$$
\left.\frac{\partial\left(a_{0}, a_{1}\right)}{\partial(x, y)}\right|_{(0,0)}=\left[\begin{array}{cc}
\frac{\partial a_{0}}{\partial x}(0,0) & \frac{\partial a_{0}}{\partial y}(0,0) \\
\frac{\partial a_{1}}{\partial x}(0,0) & \frac{\partial a_{1}}{\partial x}(0,0)
\end{array}\right]
$$

tem posto 1 e, admitindo $\frac{\partial a_{0}}{\partial y}(0,0) \neq 0$, pelo Teorema das Funções Implícitas temos que $y(x)$ é solução local de $a_{0}(x, y(x))=0$ para a qual, escrevendo $s(x)=a_{1}(x, y(x))$ temos que $s^{\prime}(0)=0$ e $s^{\prime \prime}(0) \neq 0$. Caso seja outro o elemento não-nulo da matriz $\left.\frac{\partial\left(a_{0}, a_{1}\right)}{\partial(x, y)}\right|_{(0,0)}$, construção análoga pode ser feita, obtendo a mesma definição. ( [1], pg. 18).
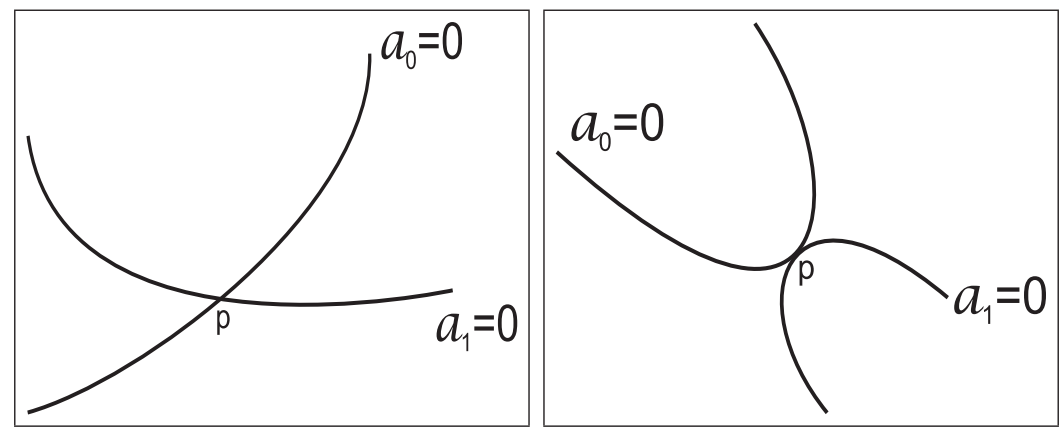

Figura 1.3: Transversalidade e Contato Quadrático das Curvas $a_{0}=0$ e $a_{1}=0$ em $\mathfrak{p}$.

Observação 1.5 ( [44], [21]). No caso de coordenadas isotérmicas, isto é, $E=G$ e $F=0$, segue que

$$
\begin{gathered}
a_{1}=-a_{3}=E^{3}\left[e_{1}^{2}+e_{2}^{2}+g_{1}^{2}+g_{2}^{2}-4\left(f_{1}^{2}+f_{2}^{2}\right)-2\left(e_{1} g_{1}+e_{2} g_{2}\right)\right], \\
a_{0}=a_{4}=-\frac{a_{2}}{6}=4 E^{3}\left[f_{1} g_{1}+f_{2} g_{2}-\left(e_{1} f_{1}+e_{2} f_{2}\right)\right] .
\end{gathered}
$$

Com isto, a equação diferencial das linhas axiais se reduz a:

$$
a_{0}(x, y)\left(d x^{4}-6 d x^{2} d y^{2}+d y^{4}\right)+a_{1}(x, y)\left(d x^{2}-d y^{2}\right) d x d y=0
$$

\subsection{Conceituação de Configurações Axiais}

Vamos denotar por $\mathcal{I}^{r}=\mathcal{I}^{r}\left(M, \mathbb{R}^{4}\right)$ o conjunto das imersões $\mathcal{C}^{r}$ de $M$ em $\mathbb{R}^{4}$. Para cada $\alpha \in \mathcal{I}^{r}$, fica bem definida a equação diferencial das linhas axiais (equação (1.4))

$$
\mathcal{G}(x, y, d x, d y)=a_{4} d y^{4}+a_{3} d y^{3} d x+a_{2} d y^{2} d x^{2}+a_{1} d y d x^{3}+a_{0} d x^{4}=0,
$$

a qual está definida no fibrado projetivo $P M$ da superfície $M$, o qual revisamos a seguir: A cada $\mathfrak{p} \in M$, está associado o círculo unitário $S_{\mathfrak{p}}^{1} \subset T_{\mathfrak{p}} M$. Neste círculo, podemos identificar cada vetor $v$ com sua antípoda $-v$, de modo que $[v]=\{v,-v\}$. Efetuando o quociente de $S_{\mathfrak{p}}^{1}$ por esta relação de equivalência, temos o espaço projetivo $P_{\mathfrak{p}} M$. Deno- 
tando por $v$ o representante da classe, temos

$$
P M:=\left\{(\mathfrak{p}, v): \mathfrak{p} \in M, v \in P_{\mathfrak{p}} M\right\}
$$

O fibrado projetivo $(P M, M, \pi)$, sendo $\pi$ a projeção $\pi(\mathfrak{p}, v)=\mathfrak{p}$, tem estrutura de variedade diferenciável: para cada aberto $\mathcal{V} \subset M$, estão definidas duas cartas $\left(x, y, \frac{d y}{d x}\right)$ e $\left(x, y, \frac{d x}{d y}\right)$ as quais cobrem $\pi^{-1}(\mathcal{V})$.

Definição 1.6. Para cada $\alpha \in \mathcal{I}^{r}$, definimos a Superfície de Lie-Cartan da imersão $\alpha$ por $\mathbb{L}_{\alpha}:=\mathcal{G}_{\alpha}^{-1}(0)$.

Observação 1.7. A Superfície de Lie-Cartan que acabamos de definir é de classe $\mathcal{C}^{r-2}$ e, apesar de ser regular sobre $\left(M \backslash \mathcal{U}_{\alpha}\right)$, pode apresentar singularidades sobre $\mathcal{U}_{\alpha}$. Além disso, como a equação (1.8) é uma equação quártica e fica indefinida sobre $\mathcal{U}_{\alpha}$, temos que a superfície $\mathbb{L}_{\alpha}$ recobre 4 vezes $\left(M \mathcal{U}_{\alpha}\right)$ e contém o eixo projetivo $\pi^{-1}(\mathfrak{p})$ sobre cada $\mathfrak{p} \in \mathcal{U}_{\alpha}$.

Na carta $(x, y, p), \operatorname{com} p=\frac{d y}{d x}$, a equação (1.8) é descrita por:

$$
\mathcal{G}(x, y, p)=a_{4} p^{4}+a_{3} p^{3}+a_{2} p^{2}+a_{1} p+a_{0}=0 .
$$

Consideremos o campo $X_{\alpha}$, de classe $\mathcal{C}^{r-3}$, conhecido como Campo de Lie-Cartan,

$$
X_{\alpha}:=\mathcal{G}_{p} \frac{\partial}{\partial x}+p \mathcal{G}_{p} \frac{\partial}{\partial y}-\left(\mathcal{G}_{x}+p \mathcal{G}_{y}\right) \frac{\partial}{\partial p} .
$$

Este campo é tangente à superfície de Lie-Cartan, visto que $\langle\nabla \mathcal{G}, X\rangle=\mathcal{G}_{x} \cdot \mathcal{G}_{p}+p \mathcal{G}_{y}$. $\mathcal{G}_{p}-\mathcal{G}_{x} \cdot \mathcal{G}_{p}-p \mathcal{G}_{y} \cdot \mathcal{G}_{p}=0$.

Observação 1.8. Na carta $(x, y, q)$, onde $q=\frac{d x}{d y}$, a equação das linhas axiais é descrita por

$$
a_{4}+a_{3} q+a_{2} q^{2}+a_{1} q^{3}+a_{0} q^{4}=0
$$

e o Campo de Lie-Cartan tem a expressão

$$
Y_{\alpha}:=q \mathcal{G}_{q} \frac{\partial}{\partial x}+\mathcal{G}_{q} \frac{\partial}{\partial y}-\left(q \mathcal{G}_{x}+\mathcal{G}_{y}\right) \frac{\partial}{\partial p}
$$

Definimos as Linhas de Curvatura Axial como a projeção, por $\pi: P M \longrightarrow M$, das linhas integrais $\mathcal{L}_{\alpha}$ do campo de Lie-Cartan $X_{\alpha}$, conforme ilustramos na Figura 1.4. Sobre $\mathfrak{p} \in\left(M \backslash \mathcal{U}_{\alpha}\right)$ temos 4 direções bem definidas, que são soluções da equação (1.9). Duas delas estão associadas ao eixo maior e duas ao eixo menor da elipse de curvatura $\varepsilon_{\alpha}(\mathfrak{p})$. A projeção nos dá então dois campos de pares de linhas tangentes ortogonais, cada par 
chamado de uma rede integral, sendo uma rede associada ao eixo menor e outra ao eixo maior. À rede associada ao eixo maior daremos o nome de Linhas de Curvatura Axial Principal e à rede associada ao eixo menor daremos o nome de Linhas de Curvatura Axial Média.

Temos, portanto, duas Configurações Axiais: a Configuração Axial Principal $\mathcal{P}_{\alpha}=$ $\left\{\mathcal{U}_{\alpha}, \mathcal{X}_{\alpha}\right\}$ definida pelos pontos axiumbílicos $\mathcal{U}_{\alpha}$ e pela rede integral $\mathcal{X}_{\alpha}$, em $\left(M \backslash \mathcal{U}_{\alpha}\right)$, na qual a imersão é curvada ao longo dos extremos do eixo maior da elipse de curvatura, e a Configuração Axial Média $\mathcal{Q}_{\alpha}=\left\{\mathcal{U}_{\alpha}, \mathcal{Y}_{\alpha}\right\}$ definida pelos pontos axiumbílicos $\mathcal{U}_{\alpha}$ e a rede integral $\mathcal{Y}_{\alpha}$, em $\left(M \backslash \mathcal{U}_{\alpha}\right)$, na qual a imersão é curvada ao longo dos extremos do eixo menor da elipse de curvatura.

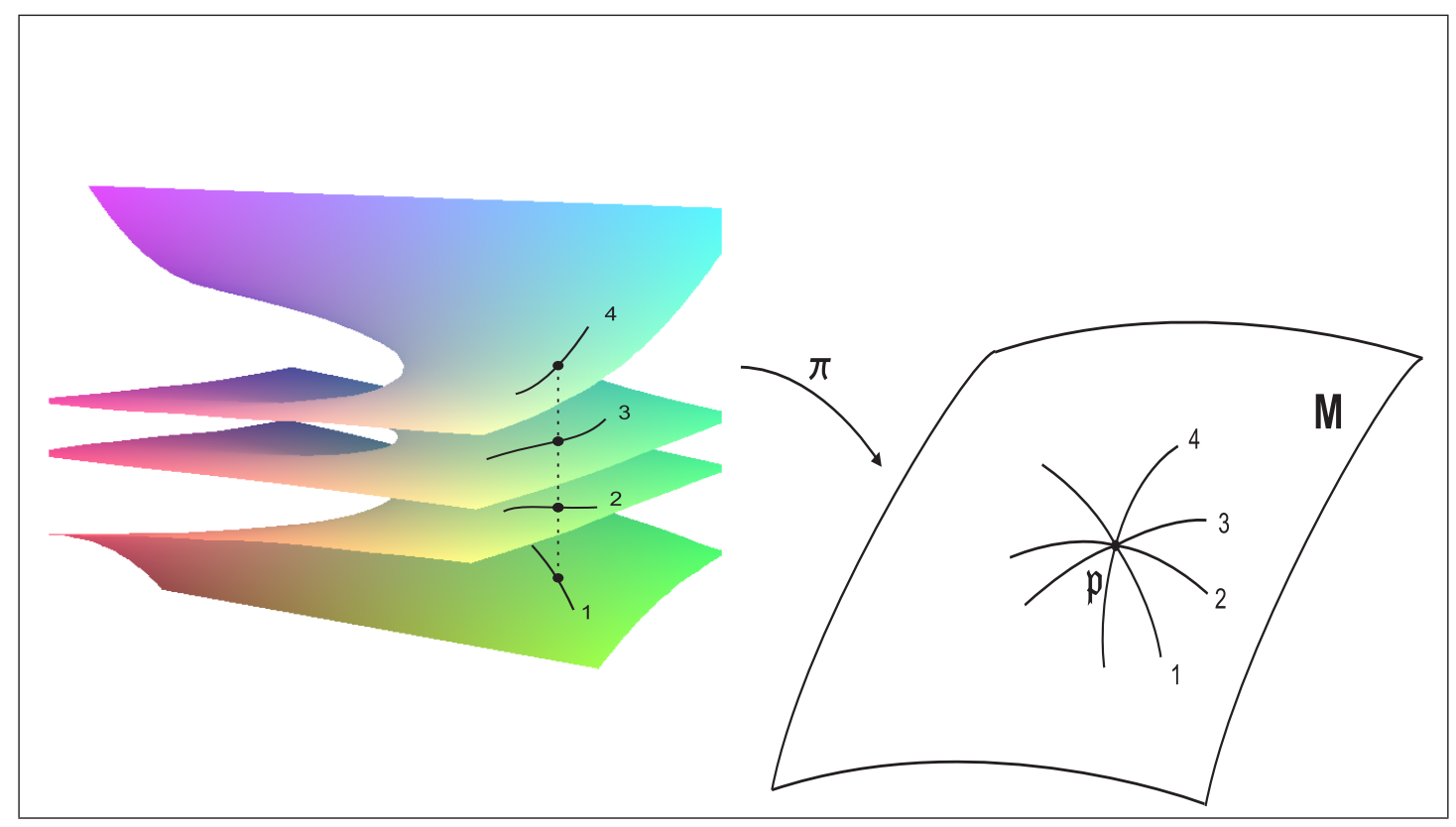

Figura 1.4: Ilustração da projeção em $M$ das linhas $\mathcal{L}_{\alpha}$ do campo de Lie-Cartan na vizinhança de um ponto $\mathfrak{p} \in\left(M \mathcal{U}_{\alpha}\right)$. Os números ilustram a projeção de cada linha, temos portanto as cruzes determinadas por $1-3$ e $2-4$. 


\subsection{Equação Diferencial em uma Carta de Monge}

Vamos considerar a expressão local da superfície por meio de uma carta de Monge, ou seja, tomando um ponto $\mathfrak{p}$ na superfície $M$, o qual será a origem de nosso sistema de coordenadas, parametrizamos uma vizinhança deste ponto da forma

$$
\left\{\begin{array}{c}
z=R(x, y), \\
w=S(x, y),
\end{array}\right.
$$

onde

$$
\begin{aligned}
R(x, y) & =\frac{r_{20}}{2} x^{2}+r_{11} x y+\frac{r_{02}}{2} y^{2}+\frac{r_{30}}{6} x^{3}+\frac{r_{21}}{2} x^{2} y+\frac{r_{12}}{2} x y^{2}+\frac{r_{03}}{6} y^{3} \\
& +\frac{r_{40}}{24} x^{4}+\frac{r_{31}}{6} x^{3} y+\frac{r_{22}}{4} x^{2} y^{2}+\frac{r_{13}}{6} x y^{3}+\frac{r_{04}}{24} y^{4}+\text { h.o.t. }, \\
S(x, y) & =\frac{s_{20}}{2} x^{2}+s_{11} x y+\frac{s_{02}}{2} y^{2}+\frac{s_{30}}{6} x^{3}+\frac{s_{21}}{2} x^{2} y+\frac{s_{12}}{2} x y^{2}+\frac{s_{03}}{6} y^{3} \\
& +\frac{s_{40}}{24} x^{4}+\frac{s_{31}}{6} x^{3} y+\frac{s_{22}}{4} x^{2} y^{2}+\frac{s_{13}}{6} x y^{3}+\frac{s_{04}}{24} y^{4}+\text { h.o.t. }
\end{aligned}
$$

Assim, no ponto $(x, y, R(x, y), S(x, y))$, o plano tangente à superfície neste ponto é gerado por $\left\{t_{1}, t_{2}\right\}$, onde $t_{1}=\left(1,0, R_{x}, S_{x}\right)$ e $t_{2}=\left(0,1, R_{y}, S_{y}\right)$. O plano normal é então gerado pelos vetores $\left\{N_{1}, N_{2}\right\}$, onde $N_{1}=\frac{\widetilde{N_{1}}}{\left|\widetilde{N_{1}}\right|}$ e $N_{2}=\frac{\widetilde{N_{2}}}{\left|\widetilde{N_{2}}\right|}$, sendo $\widetilde{N_{1}}=\left(-R_{x},-R_{y}, 1,0\right)$ e $\widetilde{N_{2}}=t_{1} \wedge t_{2} \wedge \widetilde{N_{1}}$. Aqui $\wedge$ é o produto vetorial de três vetores em $\mathbb{R}^{4}$, definido a partir da equação

$$
\operatorname{det}\left(t_{1}, t_{2}, \widetilde{N_{1}}, \bullet\right)=\left\langle\widetilde{N_{2}}, \bullet\right\rangle
$$

Sendo $R$ e $S$ dadas a partir de (1.11) e (1.12), temos

$$
E=1+O(2), \quad F=O(2), \quad G=1+O(2)
$$

e

$$
\begin{array}{ll}
e_{1}=r_{20}+r_{30} x+r_{21} y+O(2), & e_{2}=s_{20}+s_{30} x+s_{21} y+O(2), \\
f_{1}=r_{11}+r_{21} x+r_{12} y+O(2), & f_{2}=s_{11}+s_{21} x+s_{12} y+O(2), \\
g_{1}=r_{02}+r_{12} x+r_{03} y+O(2), & g_{2}=s_{02}+s_{12} x+s_{03} y+O(2) .
\end{array}
$$

De posse dos coeficientes da primeira e segunda formas fundamentais, podemos determinar a equação diferencial das linhas axiais em termos da carta de Monge $(x, y, R(x, y), S(x, y))$. Conforme a Proposição 1.4, os axiumbílicos são determinados pelas interseções das curvas $a_{0}(x, y)=0$ e $a_{1}(x, y)=0$. Na vizinhança de $(0,0)$, temos

$$
a_{0}(x, y)=a_{00}^{0}+a_{10}^{0} x+a_{01}^{0} y+O(2)
$$


e

$$
a_{1}(x, y)=a_{00}^{1}+a_{10}^{1} x+a_{01}^{1} y+O(2)
$$

sendo

$$
\begin{aligned}
& a_{00}^{0}=r_{11}\left(r_{02}-r_{20}\right)+s_{11}\left(s_{02}-s_{20}\right), \\
& a_{10}^{0}=r_{21}\left(r_{02}-r_{20}\right)+r_{11}\left(r_{12}-r_{30}\right)+s_{11}\left(s_{12}-s_{30}\right)+s_{21}\left(s_{02}-s_{20}\right), \\
& a_{01}^{0}=r_{12}\left(r_{02}-r_{20}\right)+r_{11}\left(r_{03}-r_{21}\right)+s_{11}\left(s_{03}-s_{21}\right)+s_{12}\left(s_{02}-s_{20}\right)
\end{aligned}
$$

e

$$
\begin{aligned}
& a_{00}^{1}=\left(r_{02}-r_{20}\right)^{2}+\left(s_{02}-s_{20}\right)^{2}-4\left(r_{11}^{2}+s_{11}^{2}\right), \\
& a_{10}^{1}=2\left(r_{12}-r_{30}\right)\left(r_{02}-r_{20}\right)+2\left(s_{12}-s_{30}\right)\left(s_{02}-s_{20}\right)-8\left(r_{21} r_{11}+s_{21} s_{11}\right), \\
& a_{01}^{1}=2\left(r_{03}-r_{21}\right)\left(r_{02}-r_{20}\right)+2\left(s_{03}-s_{21}\right)\left(s_{02}-s_{20}\right)-8\left(r_{12} r_{11}+s_{12} s_{11}\right) .
\end{aligned}
$$

Logo, a condição para o ponto $p$, expresso nessa carta de Monge por $(0,0)$, ser axiumbílico é que

$$
\left\{\begin{array}{l}
a_{00}^{0}=r_{11}\left(r_{20}-r_{02}\right)+s_{11}\left(s_{20}-s_{02}\right)=0, \\
a_{00}^{1}=4\left(r_{11}^{2}+s_{11}^{2}\right)-\left(r_{20}-r_{02}\right)^{2}-\left(s_{20}-s_{02}\right)^{2}=0 .
\end{array}\right.
$$

Manipulando algebricamente as equações acima temos, de acordo com [18], que a condição (1.15) é equivalente a

$$
\left\{\begin{array} { l } 
{ 2 r _ { 1 1 } = ( s _ { 2 0 } - s _ { 0 2 } ) , } \\
{ 2 s _ { 1 1 } = - ( r _ { 2 0 } - r _ { 0 2 } ) , }
\end{array} \quad \text { ou } \quad \left\{\begin{array}{l}
2 r_{11}=-\left(s_{20}-s_{02}\right) \\
2 s_{11}=\left(r_{20}-r_{02}\right)
\end{array}\right.\right.
$$

Observação 1.9. Se fizermos $r_{02}=r_{20}+r$ e $s_{02}=s_{20}+s, \rho^{2}=r_{11}^{2}+s_{11}^{2}$, temos que a condição para $(0,0)$ ser axiumbílico, da equação (1.15) é expressa por:

$$
\left\{\begin{array}{l}
r_{11} \cdot r+s_{11} \cdot s=0 \\
r^{2}+s^{2}=4 \rho^{2}
\end{array}\right.
$$

Ou seja, a condição de axiumbílico pode ser interpretada geometricamente pela interseção de um círculo e de uma reta no plano $(r, s)$. As interseções ocorrem quando:

$$
\left\{\begin{array} { l } 
{ r _ { 1 1 } = - \frac { s } { 2 } , } \\
{ s _ { 1 1 } = \frac { r } { 2 } , }
\end{array} \quad \text { ou } \quad \left\{\begin{array}{l}
r_{11}=\frac{s}{2}, \\
s_{11}=-\frac{r}{2},
\end{array}\right.\right.
$$

e portanto a equação (1.18) é uma outra versão da equação (1.16). 
Seja

$\alpha_{1}=s_{12}-s_{30}+2 r_{21}, \alpha_{2}=r_{30}-r_{12}+2 s_{21}, \alpha_{3}=s_{03}-s_{21}+2 r_{12}, \alpha_{4}=r_{21}-r_{03}+2 s_{12}$.

A discussão acima é sintetizada no Lema 1.10.

Lema 1.10. Se o ponto $\mathfrak{p}$, expresso na carta acima por $(0,0)$ for axiumbílico, então numa vizinhança do ponto podemos escrever a equação diferencial das linhas axiais na forma:

$$
\tilde{a}_{0}(x, y)\left(d x^{4}-6 d x^{2} d y^{2}+d y^{4}\right)+\tilde{a}_{1}(x, y)\left(d x^{2}-d y^{2}\right) d x d y+H(x, y, d x, d y)=0,
$$

onde

$$
\begin{aligned}
& \tilde{a}_{0}(x, y)=\frac{1}{2}\left(r \alpha_{1}+s \alpha_{2}\right) x+\frac{1}{2}\left(r \alpha_{3}+s \alpha_{4}\right) y+a_{20}^{0} x^{2}+a_{11}^{0} x y+a_{02}^{0} y^{2}, \\
& \tilde{a}_{1}(x, y)=2\left(s \alpha_{1}-r \alpha_{2}\right) x+2\left(s \alpha_{3}-r \alpha_{4}\right) y+a_{20}^{1} x^{2}+a_{11}^{1} x y+a_{02}^{1} y^{2}
\end{aligned}
$$

e $H$ contém os termos de ordem maior ou igual a $3 \mathrm{em}(x, y)$.

Com a notação que estamos utilizando, a condição de transversalidade das curvas $a_{0}=0$ e $a_{1}=0$ é dada por

$$
\left|\begin{array}{ll}
a_{10}^{0} & a_{01}^{0} \\
a_{10}^{1} & a_{01}^{1}
\end{array}\right| \neq 0
$$

O determinante acima vale:

$$
\left[\alpha_{2} \alpha_{3}-\alpha_{1} \alpha_{4}\right] \cdot\left(r^{2}+s^{2}\right)
$$

onde $r=r_{02}-r_{20}$ e $s=s_{02}-s_{20}$. Caso $\left(r^{2}+s^{2}\right)$ seja nulo, teremos que $a_{10}^{0}=a_{01}^{0}=$ $a_{10}^{1}=a_{01}^{1}=0$, e portanto a matriz $\left[\begin{array}{cc}a_{10}^{0} & a_{01}^{0} \\ a_{10}^{1} & a_{01}^{1}\end{array}\right]$ é identicamente nula. Assim os pontos axiumbílicos com $r=s=0$ formam um conjunto com codimensão pelo menos quatro. Portanto, a condição de transversalidade, supondo $r^{2}+s^{2} \neq 0$, é dada por

$$
T:=\alpha_{2} \alpha_{3}-\alpha_{1} \alpha_{4} \neq 0 \text {. }
$$

Admitindo a condição de transversalidade $T \neq 0$,

Lema 1.11. Considere a equação diferencial quártica

$$
\left(a_{10}^{0} x+a_{01}^{0} y\right)\left(d x^{4}-6 d x^{2} d y^{2}+d y^{4}\right)+\left(a_{10}^{1} x+a_{01}^{1} y\right) d x d y\left(d x^{2}-d y^{2}\right)=0
$$

Considere uma rotação $x=\cos \theta \cdot u+\sin \theta \cdot v, y=-\sin \theta \cdot u+\cos \theta \cdot v$, onde $\theta$ é uma 
14 CAPÍTULO 1. CONFIGURAÇÕES AXIAIS DE SUPERFÍCIES IMERSAS EM $\mathbb{R}^{4}$

raiz real da equação

$-a_{01}^{0} t^{5}+\left(a_{10}^{0}-a_{01}^{1}\right) t^{4}+\left(6 a_{01}^{0}+a_{10}^{1}\right) t^{3}+\left(a_{01}^{1}-6 a_{10}^{0}\right) t^{2}-\left(a_{01}^{0}+a_{10}^{1}\right) t+a_{10}^{0}=0, t=\tan \theta$.

Então, segue que

$$
A_{01}^{0} v\left(d u^{4}-6 d u^{2} d v^{2}+d v^{4}\right)+\left(A_{10}^{1} u+A_{01}^{1} v\right) d u d v\left(d u^{2}-d v^{2}\right)=0,
$$

sendo

$A_{01}^{0}=A_{01}^{0}\left(a_{10}^{0}, a_{01}^{0}, a_{10}^{1}, a_{01}^{1}, \theta\right)$,

$A_{10}^{1}=A_{10}^{1}\left(a_{10}^{0}, a_{01}^{0}, a_{10}^{1}, a_{01}^{1}, \theta\right) e$

$A_{01}^{1}=A_{01}^{1}\left(a_{10}^{0}, a_{01}^{0}, a_{10}^{1}, a_{01}^{1}, \theta\right)$.

Demonstração: Fazendo $x=\cos \theta \cdot u+\sin \theta \cdot v, y=-\sin \theta \cdot u+\cos \theta \cdot v$, segue que $d x=\cos \theta \cdot d u+\sin \theta \cdot d v, d y=-\sin \theta \cdot d u+\cos \theta \cdot d v$. Substiuindo na Equação 1.22, temos:

$$
\begin{gathered}
-a_{01}^{0} \sin ^{5} \theta+\left(a_{10}^{0}-a_{01}^{1}\right) \sin ^{4} \theta \cos \theta+\left(6 a_{01}^{0}+a_{10}^{1}\right) \sin ^{3} \theta \cos ^{2} \theta+ \\
\left(a_{01}^{1}-6 a_{10}^{0}\right) \sin ^{2} \theta \cos ^{3} \theta-\left(a_{01}^{0}+a_{10}^{1}\right) \sin \theta \cos ^{4} \theta+a_{10}^{0} \cos ^{5} \theta=0,
\end{gathered}
$$

Dividindo por $\cos ^{5} \theta$, obtemos a forma

$-a_{01}^{0} t^{5}+\left(a_{10}^{0}-a_{01}^{1}\right) t^{4}+\left(6 a_{01}^{0}+a_{10}^{1}\right) t^{3}+\left(a_{01}^{1}-6 a_{10}^{0}\right) t^{2}-\left(a_{01}^{0}+a_{10}^{1}\right) t+a_{10}^{0}=0, t=\tan \theta$.

Assim, para tal valor de $\theta$, resulta:

$$
A_{01}^{0} v\left(d u^{4}-6 d u^{2} d v^{2}+d v^{4}\right)+\left(A_{10}^{1} u+A_{01}^{1} v\right) d u d v\left(d u^{2}-d v^{2}\right)=0 .
$$

Observe que quando $a_{01}^{0}=0$ uma rotação de ângulo $\pi / 2$ é suficiente para obter o resultado estabelecido.

Proposição 1.12. Seja $\mathfrak{p}$ um ponto axiumbílico. Então existe uma carta de Monge e uma homotetia em $\mathbb{R}^{4}$ tal que a equação diferencial das linhas axiais é dada por:

$$
y\left(d y^{4}-6 d x^{2} d y^{2}+d x^{4}\right)+(a x+b y) d x d y\left(d x^{2}-d y^{2}\right)+H(x, y, d x, d y)=0,
$$

onde $H$ contém os termos de ordem maior ou igual a $2 \mathrm{em}(x, y)$. Além disso, o ponto axiumbílico $\mathfrak{p}$ é transversal se, e somente se, $a \neq 0$.

Demonstração: Considere uma parametrização $(x, y, R(x, y), S(x, y))$ dada pelas equações 


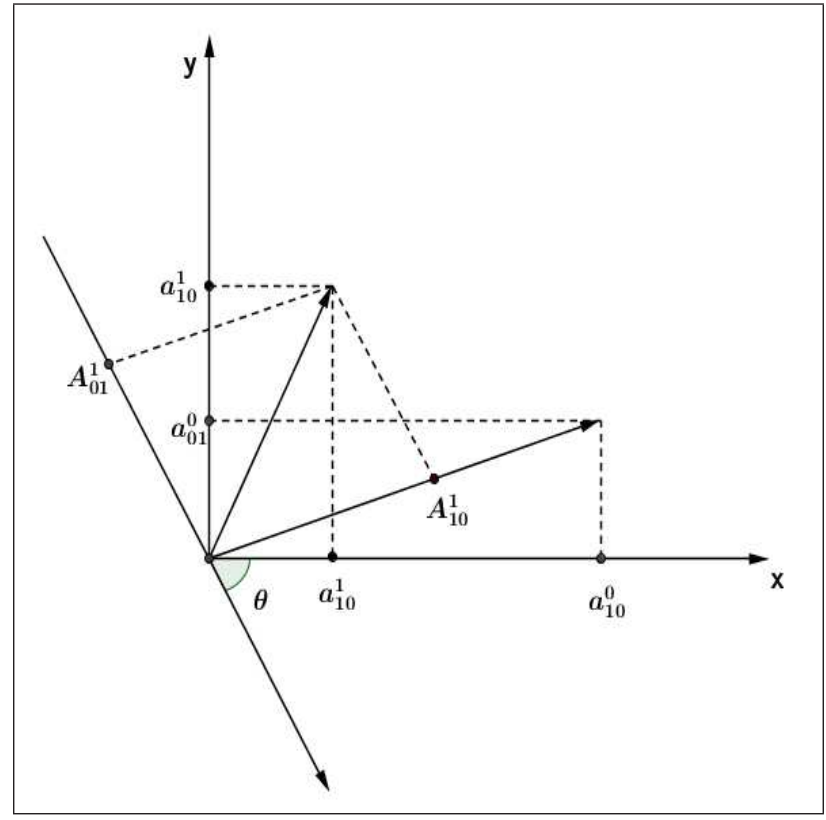

Figura 1.5: Ilustração da rotação realizada no Lema 1.11, considerando $T \neq 0$.

(1.11) e (1.12) tal que 0 é ponto axiumbílico. Pela equação (1.20) segue que:

$$
\begin{gathered}
a_{0}(x, y)=\frac{1}{2}\left(r \alpha_{1}+s \alpha_{2}\right) x+\frac{1}{2}\left(r \alpha_{3}+s \alpha_{4}\right) y+O(2), \\
a_{1}(x, y)=2\left(s \alpha_{1}-r \alpha_{2}\right) x+2\left(s \alpha_{3}-r \alpha_{4}\right) y+O(2) .
\end{gathered}
$$

Por uma rotação conveniente no plano $\{x, y\}$ dada pelo Lema 1.11 e uma homotetia em $\mathbb{R}^{4}$, é possível fazer $2 a_{10}=r \alpha_{1}+s \alpha_{2}=0$ e, quando $\left(\alpha_{1} \alpha_{4}-\alpha_{2} \alpha_{3}\right)\left(r^{2}+s^{2}\right) \neq 0$, também $a_{01}=\frac{1}{2}\left(r \alpha_{3}+s \alpha_{4}\right)=1$. Assim o resultado é estabelecido, $a=\frac{4\left(s \alpha_{1}-r \alpha_{2}\right)}{r \alpha_{3}+s \alpha_{4}}$ quando $r \alpha_{1}+s \alpha_{2}=0$ e $b=\frac{4\left(s \alpha_{3}-r \alpha_{4}\right)}{r \alpha_{3}+s \alpha_{4}}$. Se $r \neq 0$ segue $a=-\frac{4\left(r^{2}+s^{2}\right) \alpha_{2}}{r\left(r \alpha_{3}+s \alpha_{4}\right)}$ e $a=\frac{4 \alpha_{1}}{\alpha_{4}}$ quando $s \neq 0$ e $r=0$.

Observação 1.13. Se fizermos $p=\frac{d y}{d x}$, e dividindo a equação (1.23) por $d x^{4}$, obtemos a equação (1.23) no formato polinomial em $\mathfrak{p}$ :

$$
y\left(p^{4}-6 p^{2}+1\right)+(a x+b y) p\left(1-p^{2}\right)+H(x, y, p)=0,
$$

onde $H$ contém os termos de ordem maior ou igual a 2 em $(x, y)$.

Exemplo 1.14. Considere a superfície em $\mathbb{R}^{4}$ descrita pela carta de Monge $(x, y, R(x, y), S(x, y))$, onde

$$
\left\{\begin{array}{c}
R(x, y)=y^{2}-\frac{A}{96} x^{3}+\frac{B}{96} y^{3}, \\
S(x, y)=x^{2}+x y+y^{2}+\frac{1}{24} y^{3},
\end{array}\right.
$$

$\operatorname{com} A, B \in \mathbb{R}$. Na notação adotada na equação (1.11) e equação (1.12), temos que para 
este exemplo: $r_{20}=0, r_{11}=0, r_{02}=2, s_{20}=2, s_{11}=1, s_{02}=2, k=-16 A, c=16 B$, $K=4$ e todos os demais coeficientes são nulos. Nesta carta, o ponto $(0,0)$ é axiumbílico, pois

$$
\left\{\begin{array}{c}
2 r_{11}=\left(s_{20}-s_{02}\right)=0, \\
2 s_{11}=-\left(r_{20}-r_{02}\right)=2 .
\end{array}\right.
$$

Pelo Lema 1.10, a equação diferencial das linhas axiais é

$$
y\left(d y^{4}-6 d x^{2} d y^{2}+d x^{4}\right)+(A x+B y) d x d y\left(d x^{2}-d y^{2}\right)+H(x, y, d x, d y)=0,
$$

ou, usando $p=\frac{d y}{d x}$ como na Observação 1.13

$$
y\left(p^{4}-6 p^{2}+1\right)+(A x+B y) p\left(1-p^{2}\right)+H(x, y, p)=0
$$

onde, tanto na equação (1.27) quanto na equação (1.28), $H$ contém os termos de ordem maior ou igual a 2 em $(x, y)$, conforme cada caso. A condição de transversalidade é dada por $A \neq 0$.

Observação 1.15. Pelas operações que fizemos nesta seção, verificamos que toda carta de Monge com origem do sistema de coordenadas sendo um ponto axiumbílico tem a equação diferencial das linhas axiais do tipo descrito pela equação (1.24). Então, toda imersão tem, na vizinhança de um ponto axiumbílico, a equação diferencial das linhas axiais com parte linear do tipo de uma equação (1.28). Portanto, as configurações axiais na vizinhança de um ponto axiumbílico são sempre equivalentes às configurações axiais de uma carta do tipo descrito na equação (1.25). 


\section{Capítulo 2}

\section{Pontos Axiumbílicos Estáveis}

Vamos considerar a vizinhança de um ponto axiumbílico $\mathfrak{p}$, o qual é determinado pela interseção transversal das curvas $a_{0}=0$ e $a_{1}=0$ (veja equação (1.5)). Descrevendo uma vizinhança de $\mathfrak{p}$ numa carta de Monge conforme a Seção 1.3, temos pela Proposição 1.12 e Observação 1.13 que a equação diferencial das linhas axiais é descrita por

$$
\mathcal{G}(x, y, p)=y\left(p^{4}-6 p^{2}+1\right)+(a x+b y) p\left(1-p^{2}\right)+H(x, y, p)
$$

onde $H(x, y, p)$ contém os termos de ordem maior ou igual a 2 em $(x, y)$. A superfície de Lie-Cartan $\mathbb{L}_{\alpha}$ em $P M$ é dada implicitamente por

$$
\mathcal{G}(x, y, p)=0
$$

De acordo com a Observação 1.7, a superfície $\mathbb{L}_{\alpha}$ é regular e de classe $\mathcal{C}^{r-2}$ sobre $M \backslash \mathcal{U}_{\alpha}$. No caso de um ponto axiumbílico $\mathfrak{p}$ satisfazer a condição de transversalidade, temos:

Afirmação 2.1. A superfície (2.2) é regular e é de classe $\mathcal{C}^{r-2}$ na vizinhança do eixo projetivo $p$.

Prova: De fato, o gradiente de $\mathcal{G}$ nos pontos $(0,0, p)$ é não-nulo, visto que $\mathcal{G}_{x}(0,0, p)=$ $a p\left(1-p^{2}\right), \mathcal{G}_{y}(0,0, p)=\left(p^{4}-6 p^{2}+1\right)+b p\left(1-p^{2}\right)$ e as equações $G_{x}=G_{y}=0$ não têm solução comum em $p$ (lembre que assumimos $a \neq 0$ ). Logo, pelo Teorema da Função Implícita, $\mathcal{G}^{-1}(0)$ é uma superfície regular na vizinhança do eixo projetivo.

Portanto, sobre um ponto axiumbílico transversal $\mathfrak{p}$, a regularidade da superfície $\mathbb{L}_{\alpha}$ se mantém. Consideremos, nas coordenadas $(x, y, p)$, o campo de Lie-Cartan $X$, de classe $\mathcal{C}^{r-3}$, (equação $\left.(1.10)\right)$ :

$$
X:=\mathcal{G}_{p} \frac{\partial}{\partial x}+p \mathcal{G}_{p} \frac{\partial}{\partial y}-\left(\mathcal{G}_{x}+p \mathcal{G}_{y}\right) \frac{\partial}{\partial p}
$$

cuja projeção das linhas integrais de $\left.X\right|_{\mathcal{G}=0}$ determinam as linhas de curvatura axial vizinhança de $\mathfrak{p}$ (Figura 1.4). 
Afirmação 2.2. Ao longo do eixo projetivo, o campo de Lie-Cartan é expresso por:

$$
X=-p\left[\left(p^{4}-6 p^{2}+1\right)+\left(1-p^{2}\right)(a+b p)\right] \frac{\partial}{\partial p} .
$$

Prova: Efetuando os cálculos das derivadas temos que, nos pontos $(0,0, p)$ :

$$
\left\{\begin{array}{l}
\mathcal{G}_{x}=a p\left(1-p^{2}\right) \\
\mathcal{G}_{y}=p^{4}-6 p^{2}+1+b p\left(1-p^{2}\right) \\
\mathcal{G}_{p}=y\left(4 p^{3}-12 p\right)+(a x+b y)\left(1-p^{2}\right)-2(a x+b y) p^{2}
\end{array}\right.
$$

Então:

$$
\left\{\begin{array}{l}
\mathcal{G}_{p}=y\left(4 p^{3}-12 p\right)+(a x+b y)\left(1-p^{2}\right)-2(a x+b y) p^{2}, \\
p \mathcal{G}_{p}=p\left[y\left(4 p^{3}-12 p\right)+(a x+b y)\left(1-p^{2}\right)-2(a x+b y) p^{2}\right], \\
\mathcal{G}_{x}+p \mathcal{G} y=-p\left[\left(p^{4}-6 p^{2}+1\right)+\left(1-p^{2}\right)(a+b p)\right] .
\end{array}\right.
$$

Logo, sobre o eixo projetivo, as componentes do campo de Lie-Cartan são:

$$
\left\{\begin{array}{l}
\mathcal{G}_{p}(0,0, p)=0 \\
p \mathcal{G}_{p}(0,0, p)=0 \\
\mathcal{G}_{x}+p \mathcal{G} y=-p\left[\left(p^{4}-6 p^{2}+1\right)+\left(1-p^{2}\right)(a+b p)\right]
\end{array}\right.
$$

Portanto, os equilíbrios do campo de Lie-Cartan ao longo do eixo projetivo são dados pelas raizes da equação

$$
P(p)=p R(p)=p\left[\left(p^{4}-6 p^{2}+1\right)+\left(1-p^{2}\right)(a+b p)\right]=0 .
$$

Já sabemos ser $p=0$ uma raiz de $P$, e portanto $(0,0,0)$ é uma singularidade do campo $X$. Vamos estudar o polinômio

$$
R(p)=\left(p^{4}-6 p^{2}+1\right)+\left(1-p^{2}\right)(a+b p)
$$

para obter informações com relação às outras singularidades. Com base na classificação em [27], a qual está de acordo com as seções 61, pg. 121 e 68, pg. 144 de [7], temos o seguinte lema:

Lema 2.3. Seja $f(x)=a_{0}+a_{1} x+a_{2} x^{2}+a_{3} x^{3}+a_{4} x^{4}$ um polinômio de grau 4 com coeficientes reais e seja $\Delta(f)$ o discriminante deste polinômio. Então:

i) $\Delta(f)>0 \Leftrightarrow f$ tem quatro raizes reais distintas ou quatro raizes complexas conjugadas par a par.

ii) $\Delta(f)<0 \Leftrightarrow f$ tem duas raizes reais distintas e duas raizes complexas conjugadas.

iii) $\Delta(f)=0 \Leftrightarrow f$ satisfaz alguma das seguintes condições: 
tem uma raiz real quadrupla,

tem uma raiz real tripla e uma raiz real simples,

tem duas raizes reais duplas,

tem uma raiz real dupla e duas raizes reais simples,

tem uma raiz real dupla e duas complexas conjugadas,

tem duas raizes complexas conjugadas duplas.

Para a equação (2.7), temos que:

$\Delta(R)=16 a^{5}+4\left(b^{2}+68\right) a^{4}+16\left(b^{2}+144\right) a^{3}-8\left(b^{2}-80\right)\left(16+b^{2}\right) a^{2}+96\left(16+b^{2}\right)^{2} a+4\left(16+b^{2}\right)^{3}$

$R( \pm 1)=-4, R(0)=1+a$ e $\lim _{p \longrightarrow \pm \infty} R(p)=+\infty$. Logo, $R$ tem pelo menos duas raizes reais distintas, sendo uma menor que -1 e outra maior que 1 . Resta dois casos a considerar: $\Delta(R)>0$ e $\Delta(R)<0$.

A derivada de $X$ no ponto $(0,0, p)$ é dada pela matriz

$$
D X(0,0, p)=\left[\begin{array}{ccc}
a\left(1-3 p^{2}\right) & 4 p^{3}+b\left(1-3 p^{2}\right)-12 p & 0 \\
a\left(1-3 p^{2}\right) p & p\left[4 p^{3}+b\left(1-3 p^{2}\right)-12 p\right] & 0 \\
0 & 0 & -P^{\prime}(p)
\end{array}\right]
$$

cujos autovalores são

$$
\begin{aligned}
& \lambda_{1}(p)=a\left(1-3 p^{2}\right)+p\left[4 p^{3}+b\left(1-3 p^{2}\right)-12 p\right] \\
& \lambda_{2}(p)=-P^{\prime}(p), \\
& \lambda_{3}=0 .
\end{aligned}
$$

Convém ressaltar que o eixo projetivo é o auto-espaço associado ao autovalor $\lambda_{2}$, e os auto-espaços associados aos demais autovalores são transversais ao eixo $p$. Lembrando que $P(p)=p R(p)$, temos que $P^{\prime}(p)=R(p)+p R^{\prime}(p)$. Logo, nas raizes de $R$, temos que $-P^{\prime}(p)=-p R^{\prime}(p)$. Também se $R(p)=0$, temos que

$$
\left(p^{4}-6 p^{2}+1\right)+\left(1-p^{2}\right)(a+b p)=0
$$

e, como sabemos que \pm 1 não são raizes de $R$, podemos escrever

$$
a=\frac{\left(-p^{4}+6 p^{2}-1\right)+b p\left(1-p^{2}\right)}{1-p^{2}} .
$$

Substituindo na expressão de $\lambda_{1}(p)$, resulta que, nas raizes de $R(p)$ (ou seja, nos equilíbrios 
de $X$ ao longo do eixo projetivo):

$$
\left\{\begin{array}{l}
\lambda_{1}(p)=\frac{\left(p^{2}+1\right)^{3}}{\left(p^{2}-1\right)} \\
\lambda_{2}(p)=-p R^{\prime}(p) \\
\lambda_{3}=0
\end{array}\right.
$$

Concluimos, com relação às raizes $p_{i}$, que os autovalores não-nulos são:

$$
\begin{aligned}
& p_{0}=0 \quad\left\{\begin{array}{l}
\lambda_{1}=a, \\
\lambda_{2}=-(a+1),
\end{array}\right. \\
& p_{i} \neq 0 \quad\left\{\begin{array}{l}
\lambda_{1}=\frac{\left(p_{i}^{2}+1\right)^{3}}{\left(p_{i}^{2}-1\right)} \\
\lambda_{2}=-p_{i} R^{\prime}\left(p_{i}\right),
\end{array}\right.
\end{aligned}
$$

sendo que o auto-espaço de $D X\left(0,0, p_{i}\right)$ associado a $\lambda_{1}$ é transversal ao eixo $p$ e $\lambda_{2}$ tem por auto-espaço associado o próprio eixo projetivo.

\section{$2.1 \quad$ Tipo $E_{3}$}

Considere o caso em que $a$ e $b$ são tais que $\Delta<0$. Neste caso, $R$ tem apenas duas raizes reais distintas, sendo uma delas menor que -1 e a outra maior que 1 . Neste caso, o gráfico de $R(p)$ é como na Figura 2.1.

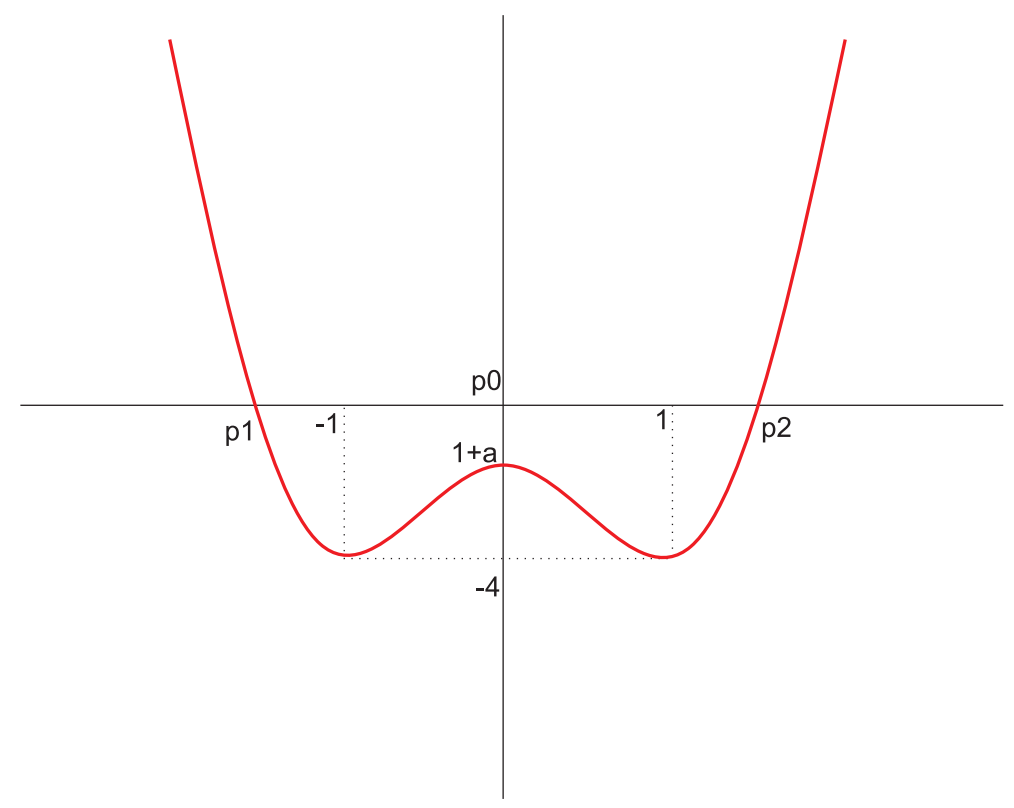

Figura 2.1: Gráfico de $R(p)$ no caso $E_{3}$.

Temos que $R(0)=1+a<0 \Rightarrow a<-1$. Logo, em (2.9), temos que $\lambda_{1}<0, \lambda_{2}>0$ e portanto $p_{0}=0$ é sela.

Com relação a $p_{1}<-1$, resulta $p_{1}^{2}-1>0$ e portanto $\lambda_{1}>0$. Veja que $R$ é decrescente em $p_{1}$ e portanto $R^{\prime}\left(p_{1}\right)<0 \Rightarrow-p_{1} R^{\prime}\left(p_{1}\right)<0$. Logo $p_{1}$ é sela. 
Quanto a $p_{2}>1$, resulta $p_{2}^{2}-1>0$ e portanto $\lambda_{1}>0$. Como $R$ e crescente em $p_{2}$, $R^{\prime}\left(p_{2}\right)>0 \Rightarrow-p_{2} R^{\prime}\left(p_{2}\right)<0$. Logo $p_{2}$ é sela.

No caso do ponto axiumbílico $E_{3}$, as singularidades são três selas hiperbólicas.

\begin{tabular}{|c|c|c|c|}
\hline & $p_{0}$ & $p_{1}$ & $p_{2}$ \\
\hline$\lambda_{1}$ & - & + & + \\
\hline$\lambda_{2}$ & + & - & - \\
\hline & sela & sela & sela \\
\hline
\end{tabular}

Tabela 2.1: Singularidades ao longo do eixo projetivo no caso $E_{3}$.

Com base nestas informações, temos uma ilustração do comportamento das curvas integrais do Campo de Lie-Cartan na vizinhança do eixo projetivo, conforme Figura 2.2.

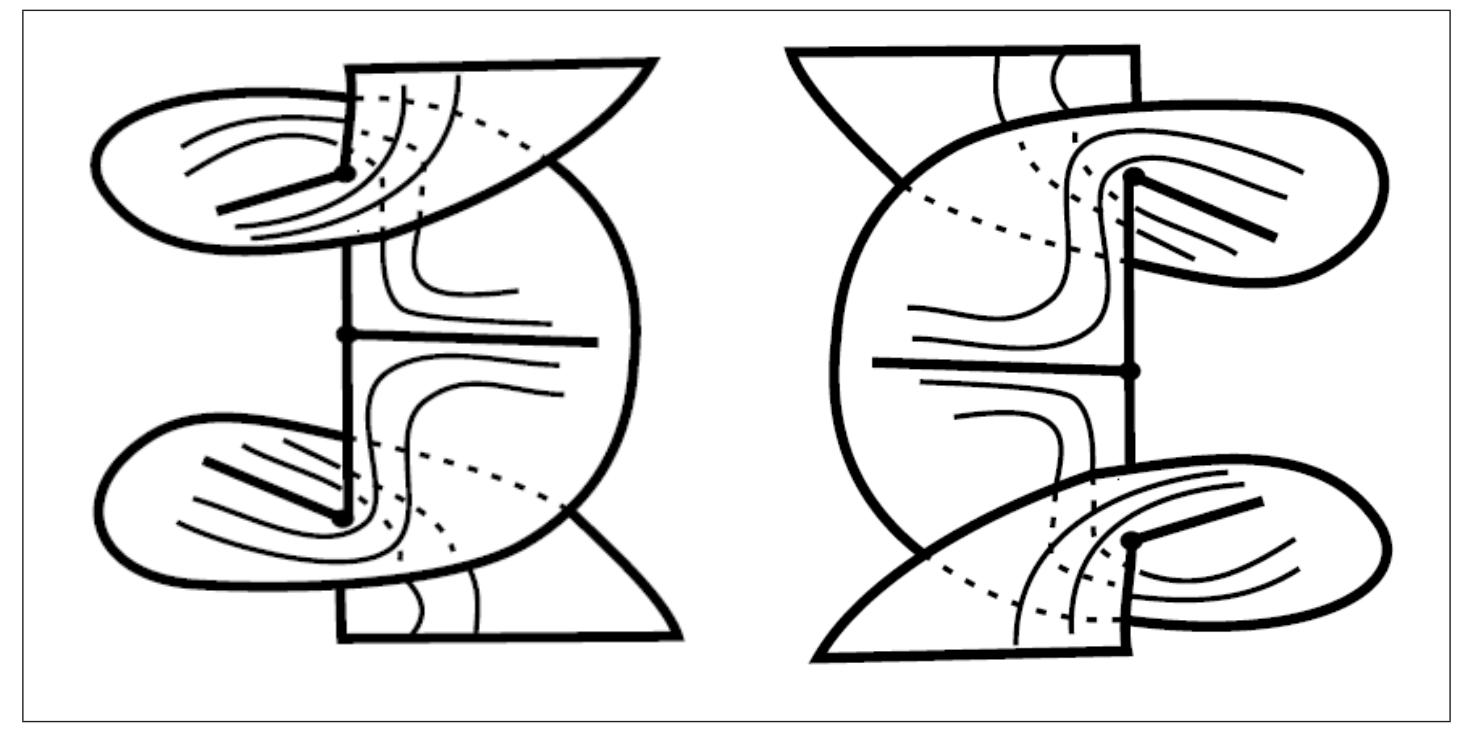

Figura 2.2: Curvas integrais de $\left.X\right|_{\mathcal{G}=0}$ na vizinhança do eixo projetivo no caso $E_{3}$.

Por meio da projeção $\pi: T M \longrightarrow M$, temos a configuração das linhas axiais na vizinhança de um ponto do tipo $E_{3}$, conforme ilustrado na Figura 2.3. 


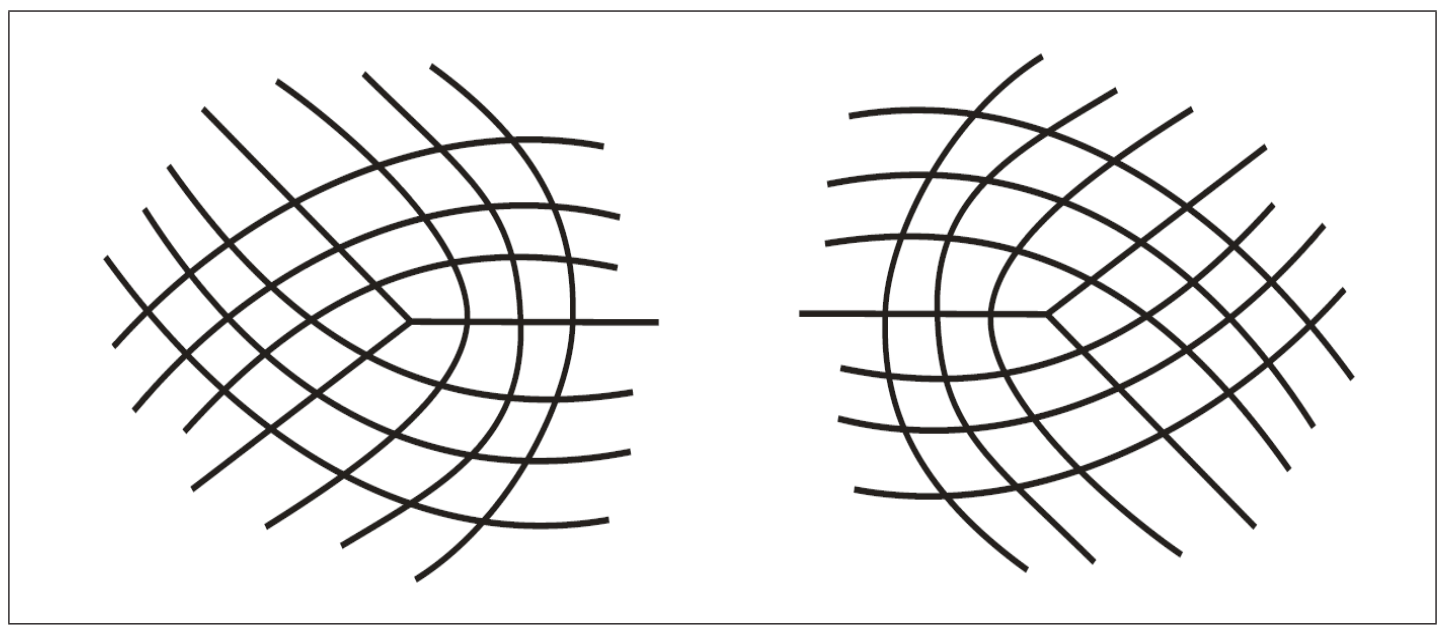

Figura 2.3: Configuração Axial Principal e Configuração Axial Média ao redor de um ponto do tipo $E_{3}$.

\subsection{Tipo $E_{4}$}

Aqui estudamos o caso em que $\Delta(a, b)>0$, sendo $a<0, a \neq-1$. Conforme o Lema 2.3 , o polinômio $R(p)$ tem quatro raizes reais distintas, $p_{1}, p_{2}, p_{3}, p_{4}$ sendo que podemos assumir $p_{1}<-1$ e $p_{4}>1$, visto que $R( \pm 1)=-4, R(0)=1+a$ e $\lim _{p \longrightarrow \pm \infty} R(p)=+\infty$. Vamos separar a análise nos casos em que $a<-1$ e $-1<a<0$.

I) $a<-1$

Aqui, temos que $R(0)=1+a<0$. Temos, ainda, dentro deste caso, quatro possíveis disposições das raizes do polinômio $R(p)$, conforme ilustramos abaixo. De acordo com as equações (2.9) e (2.10), construimos abaixo as tabelas com o sinal dos autovalores, o qual é analisado conforme o valor de $a$, a posição da raiz $p_{i}$ e a inclinação do gráfico $R^{\prime}\left(p_{i}\right)$.

i) $p_{1}<-1<p_{2}<p_{3}<p_{0}=0<1<p_{4}$

\begin{tabular}{|c|c|c|c|c|c|}
\hline & $p_{0}$ & $p_{1}$ & $p_{2}$ & $p_{3}$ & $p_{4}$ \\
\hline$\lambda_{1}$ & - & + & - & - & + \\
\hline$\lambda_{2}$ & + & - & + & - & - \\
\hline & sela & sela & sela & nó & sela \\
\hline
\end{tabular}

Tabela 2.2: Singularidades ao longo do eixo projetivo no caso $E_{4}$, caso (I.i). 


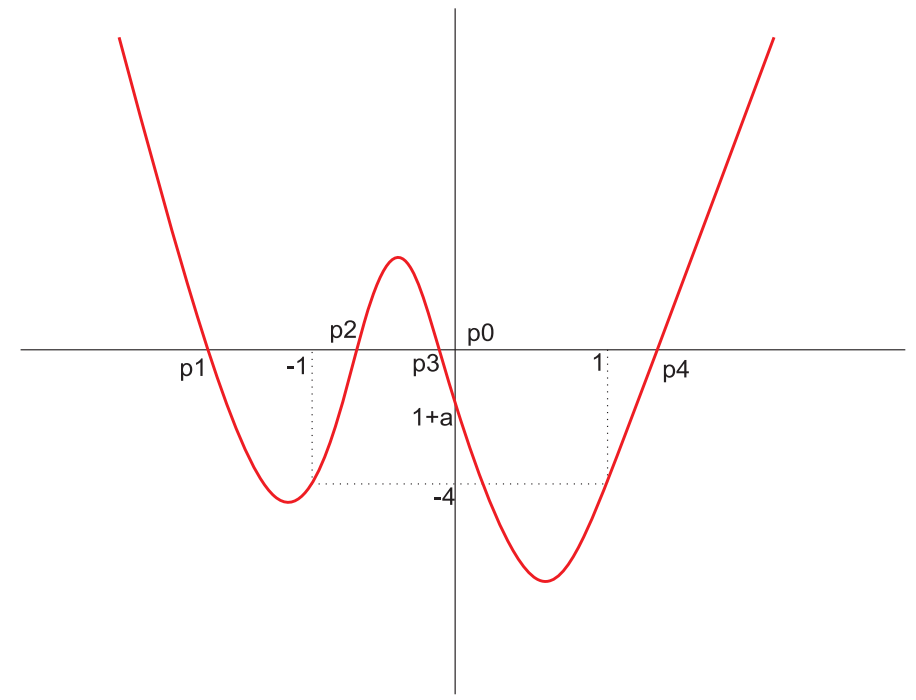

Figura 2.4: Gráfico de $R(p)$ no caso $E_{4}$, caso (I.i).

ii) $p_{1}<-1<p_{0}=0<p_{2}<p_{3}<1<p_{4}$

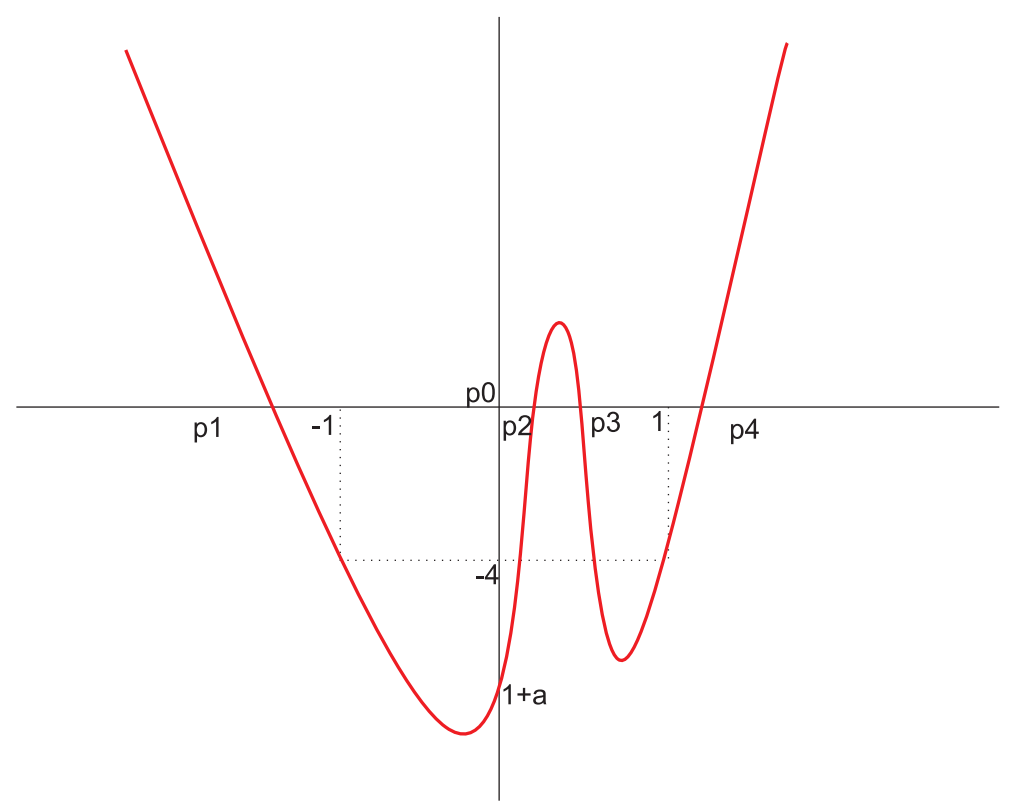

Figura 2.5: Gráfico de $R(p)$ no caso $E_{4}$, caso (I.ii). 


\begin{tabular}{|c|c|c|c|c|c|}
\hline & $p_{0}$ & $p_{1}$ & $p_{2}$ & $p_{3}$ & $p_{4}$ \\
\hline$\lambda_{1}$ & - & + & - & - & + \\
\hline$\lambda_{2}$ & + & - & - & + & - \\
\hline & sela & sela & nó & sela & sela \\
\hline
\end{tabular}

Tabela 2.3: Singularidades ao longo do eixo projetivo no caso $E_{4}$, caso (I.ii).

iii) $p_{1}<p_{2}<p_{3}<-1<p_{0}=0<1<p_{4}$

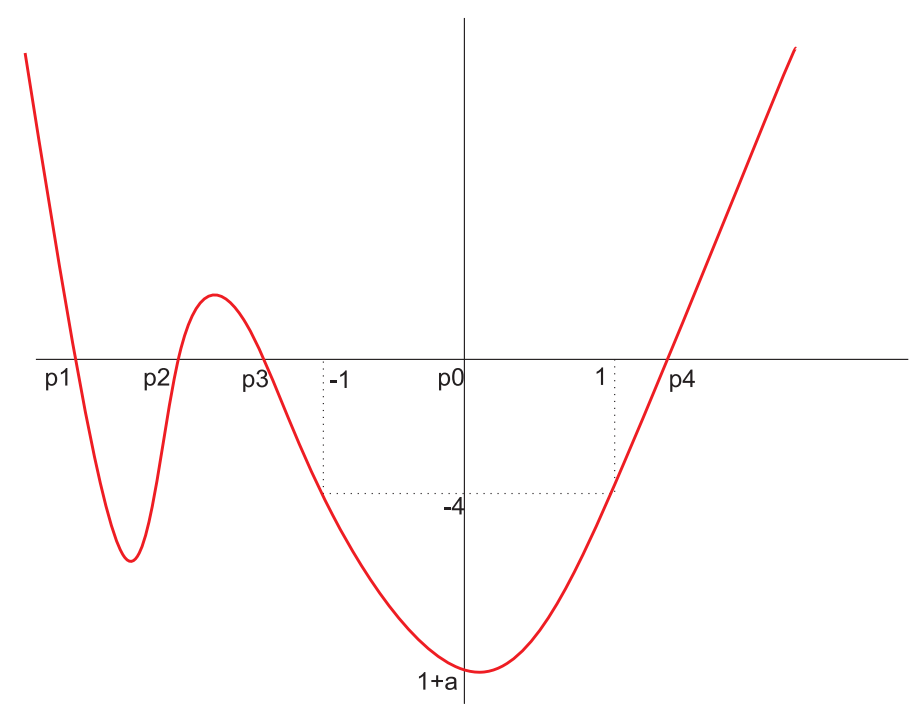

Figura 2.6: Gráfico de $R(p)$ no caso $E_{4}$, caso (I.iii).

\begin{tabular}{|c|c|c|c|c|c|}
\hline & $p_{0}$ & $p_{1}$ & $p_{2}$ & $p_{3}$ & $p_{4}$ \\
\hline$\lambda_{1}$ & - & + & + & + & + \\
\hline$\lambda_{2}$ & + & - & + & - & - \\
\hline & sela & sela & nó & sela & sela \\
\hline
\end{tabular}

Tabela 2.4: Singularidades ao longo do eixo projetivo no caso $E_{4}$, caso (I.iii). 
iv) $p_{1}<-1<p_{0}=0<1<p_{2}<p_{3}<p_{4}$

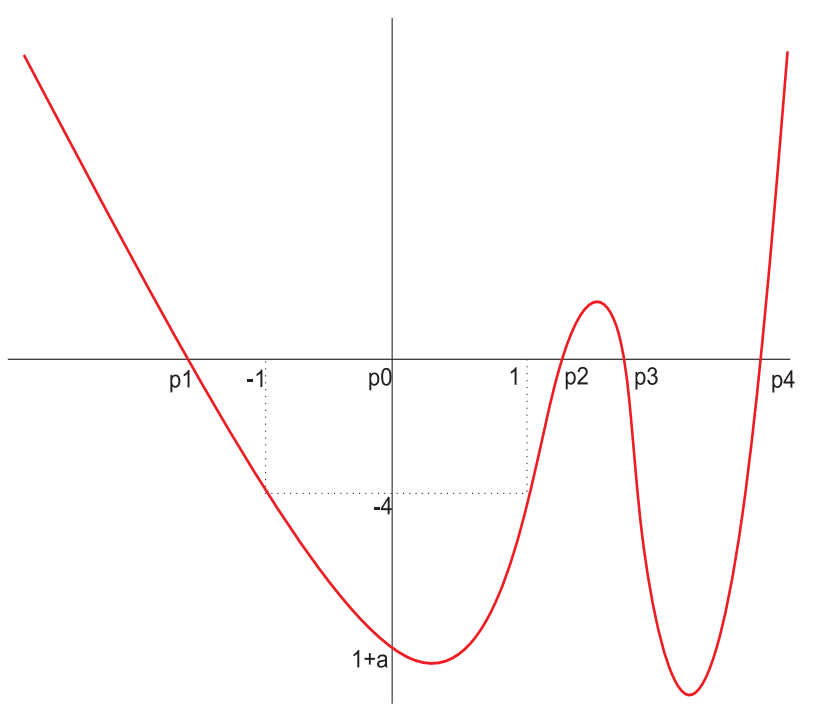

Figura 2.7: Gráfico de $R(p)$ no caso $E_{4}$, caso (I.iv).

\begin{tabular}{|c|c|c|c|c|c|}
\hline & $p_{0}$ & $p_{1}$ & $p_{2}$ & $p_{3}$ & $p_{4}$ \\
\hline$\lambda_{1}$ & - & + & + & + & + \\
\hline$\lambda_{2}$ & + & - & - & + & - \\
\hline & sela & sela & sela & nó & sela \\
\hline
\end{tabular}

Tabela 2.5: Singularidades ao longo do eixo projetivo no caso $E_{4}$, caso (I.iv). 
II) $-1<a<0$

Neste caso, $0<R(0)=1+a<1$ e com base nas equações (2.9) e (2.10), $p_{0}$ é nó, e as demais selas, conforme ilustra o gráfico do qual podemos tirar conclusões a respeito da disposição de $p_{i}$ e do sinal de $R^{\prime}\left(p_{i}\right)$.

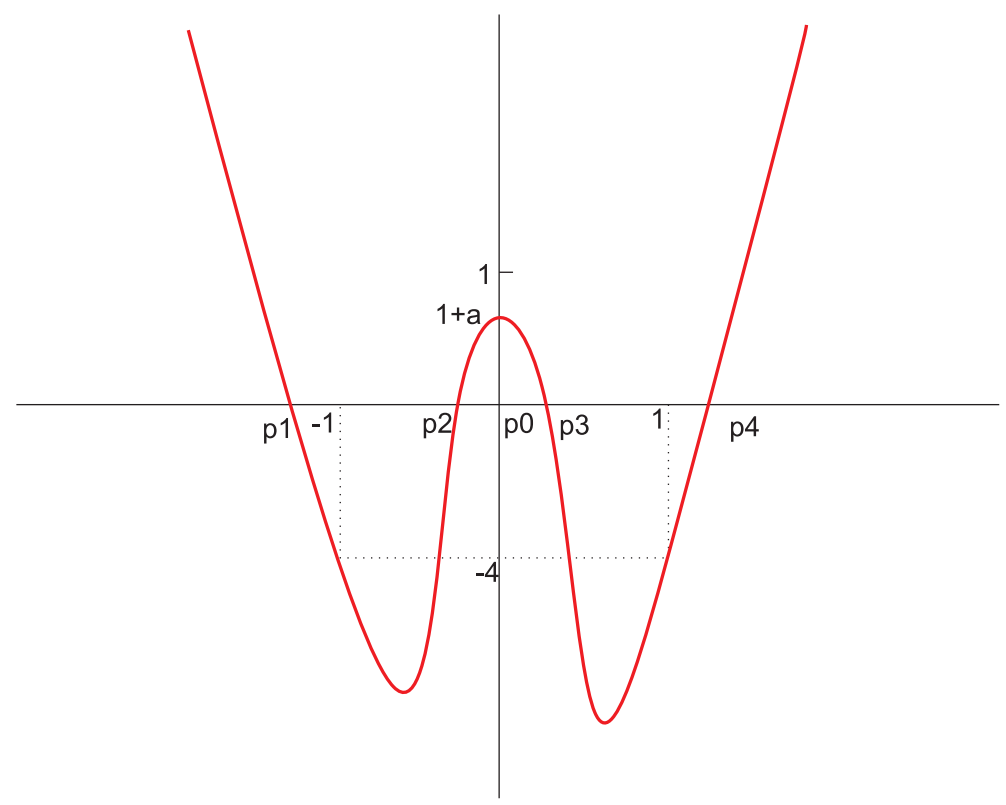

Figura 2.8: Gráfico de $R(p)$ no caso $E_{4}$, caso $(I I)$.

\begin{tabular}{|c|c|c|c|c|c|}
\hline & $p_{0}$ & $p_{1}$ & $p_{2}$ & $p_{3}$ & $p_{4}$ \\
\hline$\lambda_{1}$ & - & + & - & - & + \\
\hline$\lambda_{2}$ & - & - & + & + & - \\
\hline & nó & sela & sela & sela & sela \\
\hline
\end{tabular}

Tabela 2.6: Singularidades ao longo do eixo projetivo no caso $E_{4}$, caso $(I I)$. 


\section{2. $\mathrm{TIPO} \mathrm{E}_{4}$}

Em todos os casos apresentados, temos quatro singularidades do tipo sela e uma do tipo nó e todos estes casos apresentam as mesmas configurações axiais. Seja $p_{0}=0 \mathrm{a}$ singularidade do tipo nó, como no caso $(I I)$. Ilustramos na Figura 2.9 as curvas integrais do Campo de Lie-Cartan na vizinhança do eixo projetivo.

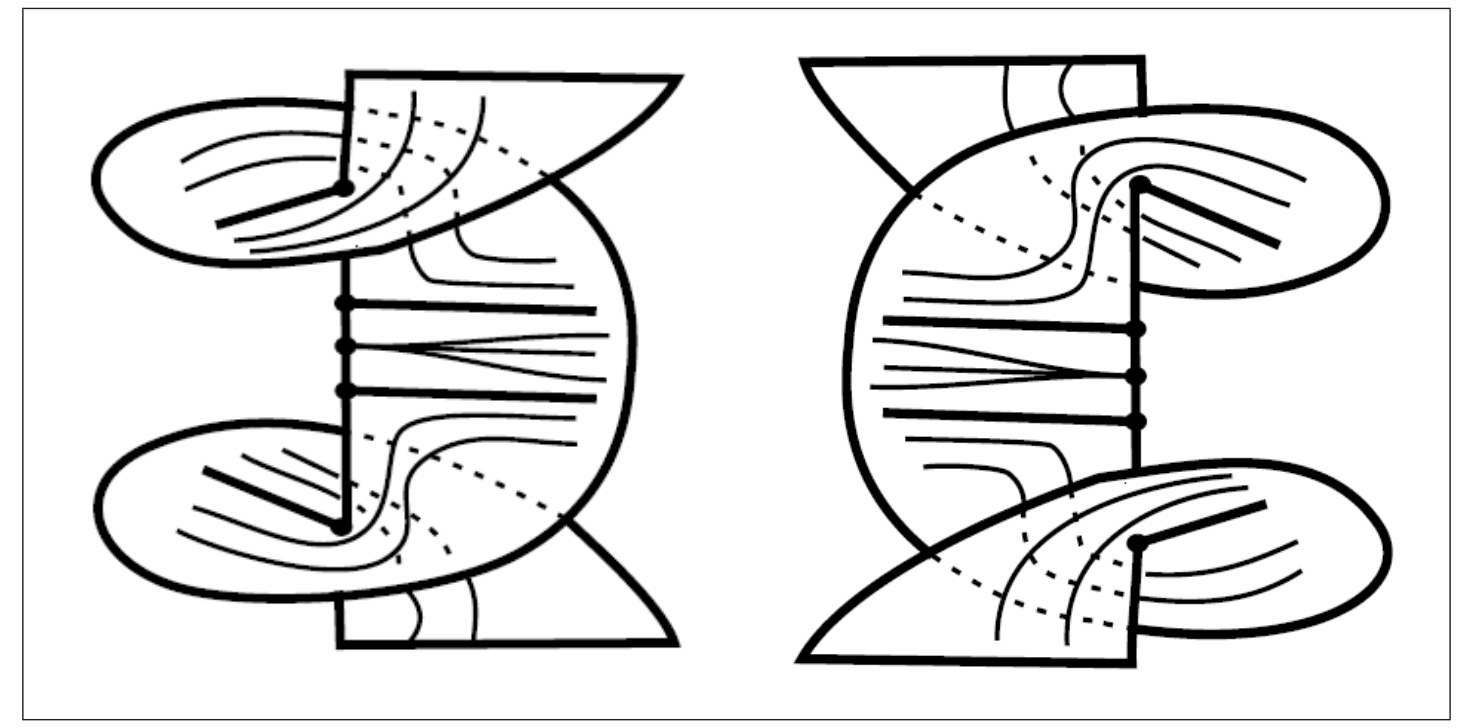

Figura 2.9: Curvas integrais de $\left.X\right|_{\mathcal{G}}=0$ na vizinhança do eixo projetivo no caso $E_{4}$.

Efetuando a projeção temos então as configurações axiais ilustradas na Figura 2.10.

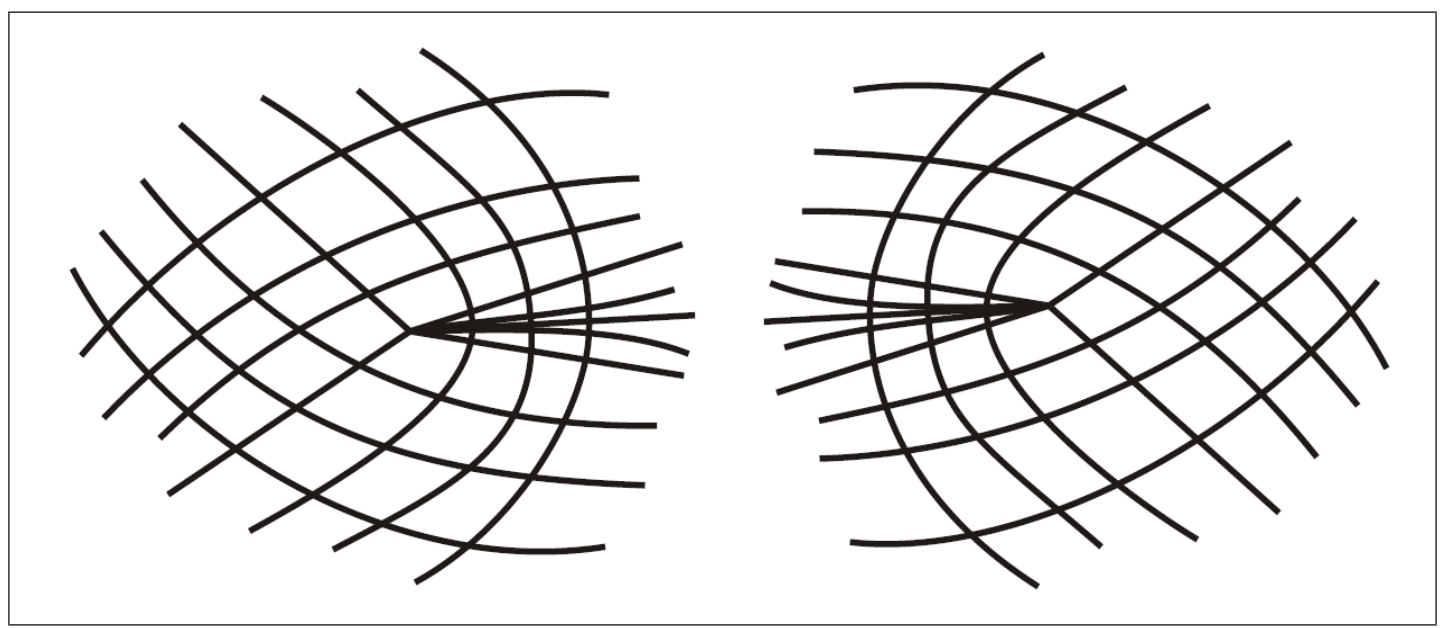

Figura 2.10: Configuração Axial Principal e Configuração Axial Média ao redor de um ponto do tipo $E_{4}$. 


\section{$2.3 \quad$ Tipo $E_{5}$}

Neste caso, temos $\Delta(a, b)>0$ com $a>0$. Novamente, o polinômio $R(p)$ tem quatro raizes reais distintas, e temos que $R(0)=1+a>1$. Conforme considerações anteriores, duas de suas raizes são em módulo maior que 1 , digamos $p_{1}<-1$ e $p_{4}>1$. Como $R( \pm 1)=-4$ e $R(0)>1$, resulta da continuidade de $R$ que as outras duas raizes serão em módulo menor do que 1 , consideremos $-1<p_{2}<0<p_{3}<-1$. Assim, a disposição das raizes são da forma $p_{1}<-1<p_{2}<p_{0}=0<p_{3}<1<p_{4}$. A Figura 2.11 ilustra tal situação.

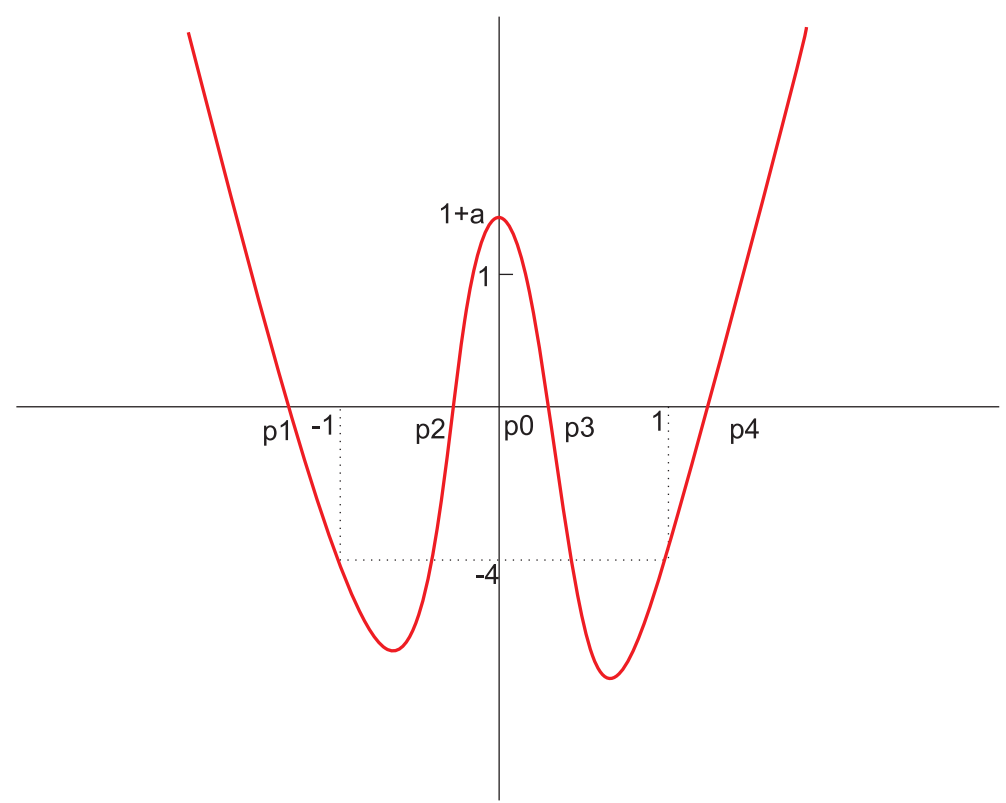

Figura 2.11: Gráfico de $R(p)$ no caso $E_{5}$.

Analisando, nas raizes $p_{i}$, os autovalores conforme as equações (2.9) e (2.10), temos a tabela:

\begin{tabular}{|c|c|c|c|c|c|}
\hline & $p_{0}$ & $p_{1}$ & $p_{2}$ & $p_{3}$ & $p_{4}$ \\
\hline$\lambda_{1}$ & + & + & - & - & + \\
\hline$\lambda_{2}$ & - & - & + & + & - \\
\hline & sela & sela & sela & sela & sela \\
\hline
\end{tabular}

Tabela 2.7: Singularidades ao longo do eixo projetivo no caso $E_{5}$. 
A Figura 2.12 ilustra o comportamento das curvas integrais do Campo de Lie-Cartan na vizinhança do eixo projetivo. A ilustração da superfície de Lie-Cartan no caso $E_{5}$ segue em sentido reverso da dos casos $E_{3}$ e $E_{4}$.

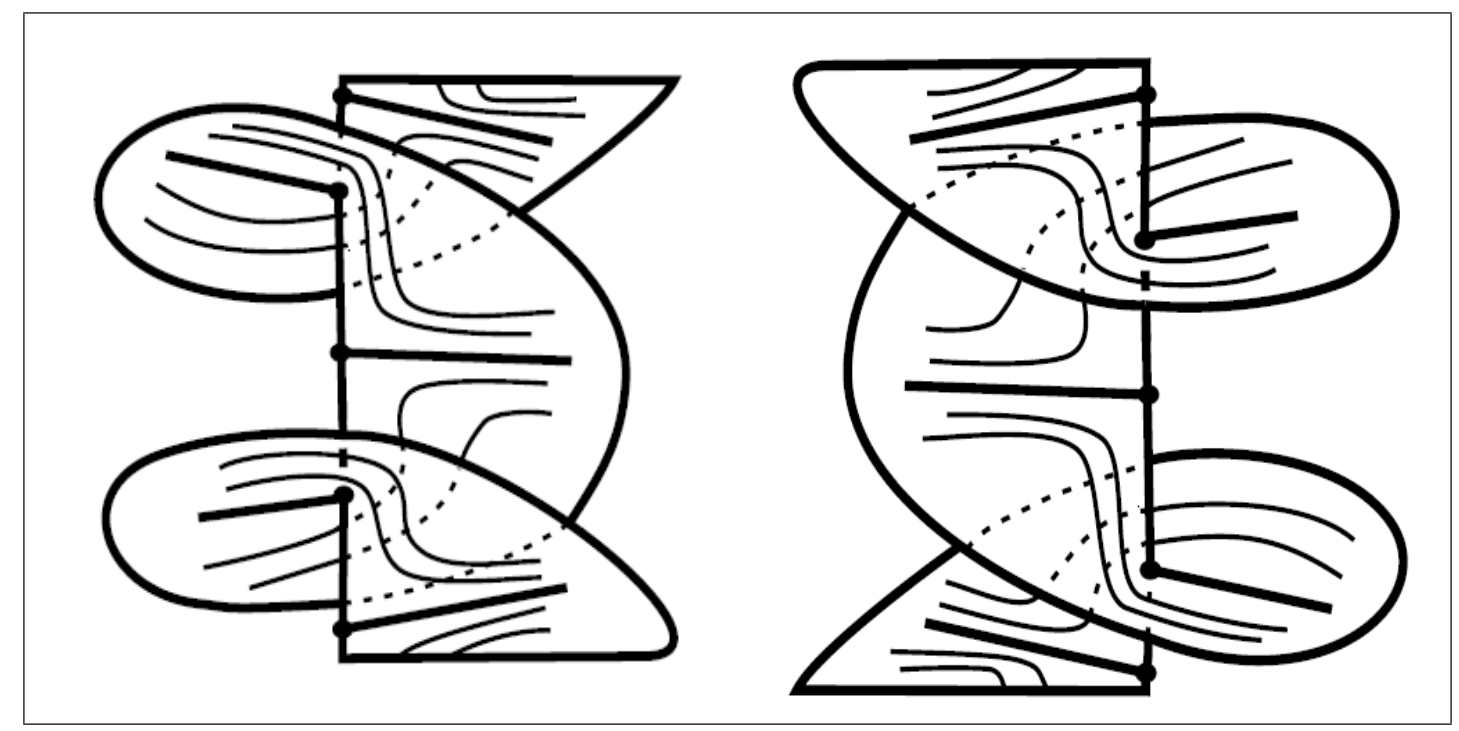

Figura 2.12: Curvas integrais de $\left.X\right|_{\mathcal{G}}=0$ na vizinhança do eixo projetivo no caso $E_{5}$.

A projeção nos dá as configurações axiais na vizinhança de um ponto do tipo $E_{5}$, conforme ilustra a Figura 2.13.

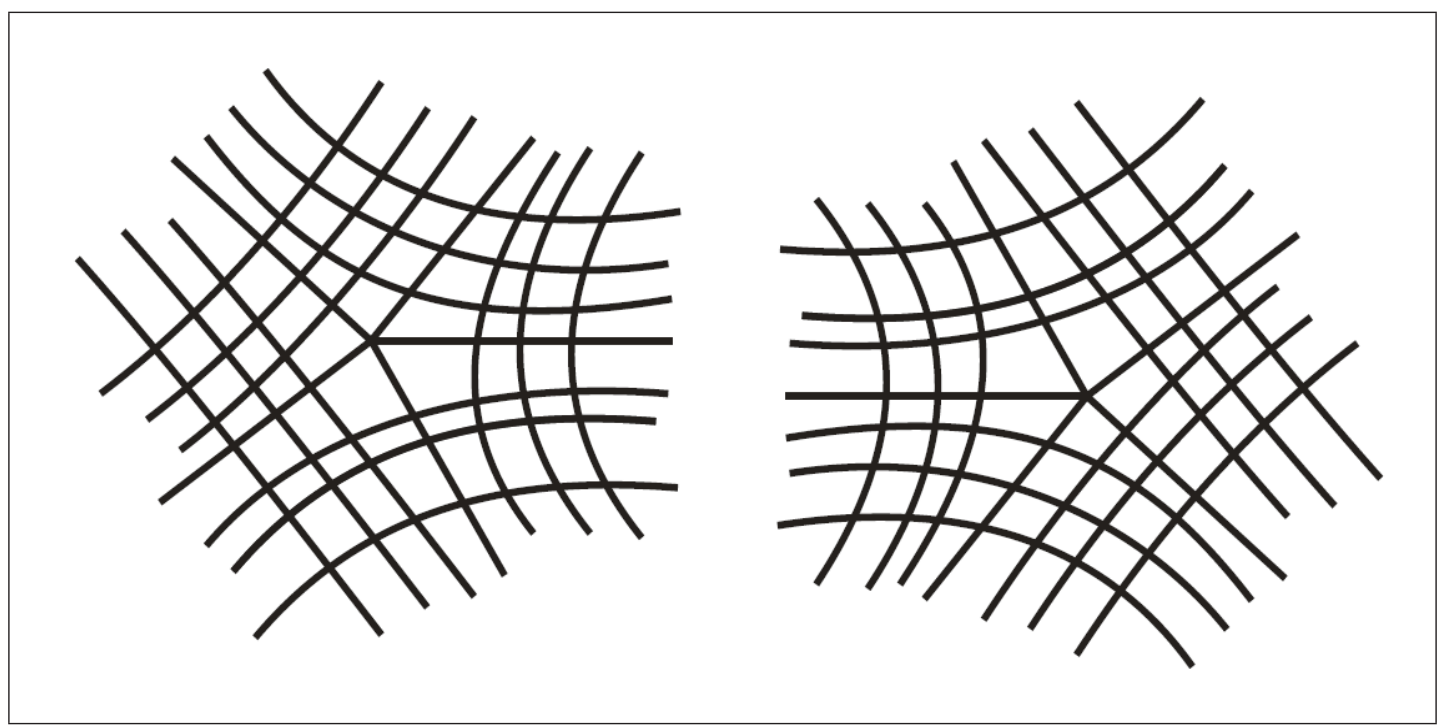

Figura 2.13: Configuração Axial Principal e Configuração Axial Média ao redor de um ponto do tipo $E_{5}$. 


\subsection{Diagrama dos Pontos Estáveis}

De fato, os pontos enunciados neste capítulo são os únicos pontos axiumbílicos que são "estruturalmente estáveis", no sentido de que as configurações axiais não se alteram por pequenas perturbações no espaço das imersões $\mathcal{I}^{r}$. Tal resultado pode ser encontrado na pg. 209 de [42]. Reunindo as informações obtidas a respeito dos pontos axiumbílicos, podemos então exibir as regiões do plano $(a, b)$ que correspondem aos tipos de pontos que observamos a seguir. Estas regiões são separadas pela curva $\Delta=0$, pela linha $a=-1 \mathrm{e}$ pela linha $a=0$, conforme ilustramos na Figura 2.14.

Teorema 2.4 ( [44], [42] p. 209). Seja $\mathfrak{p}$ um ponto axiumbílico de $\alpha \in \mathcal{I}^{r}, r \geq 5$. Então, $\alpha$ é localmente principal axial estável e localmente médio axial estável em $\mathfrak{p}$ se, e somente se $\mathfrak{p}$ é do tipo $E_{3}, E_{4}$ ou $E_{5}$.

A curva $\Delta(a, b)=0$ possui três componentes conexas, está contida na região $a \leq-1$ e é regular fora dos pontos $\left(-\frac{27}{2}, \pm \frac{5 \sqrt{5}}{2}\right)$ os quais são do tipo cúspide.

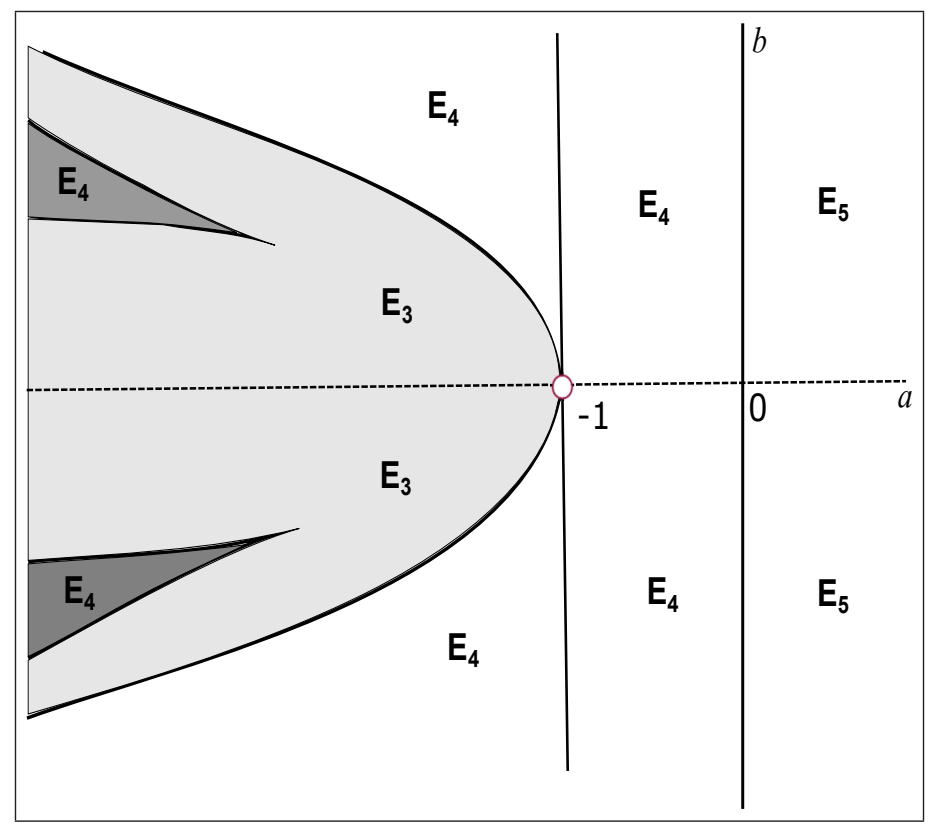

Figura 2.14: Diagrama dos Pontos Axiumbílicos Estáveis.

Demonstração: A função $\Delta(a, b)$ definida pela equação (2.8) é simétrica em $b$. Os polinômios $\Delta(a, b)$ e $\frac{\partial \Delta}{\partial b}$ na variável $b$ tem resultante igual a

$$
274877906944(1+a)\left(a^{2}+8 a+32\right)^{2} a^{16}(2 a+27)^{6} .
$$

Os pontos críticos $p_{ \pm}=\left(-\frac{27}{2}, \pm \frac{5 \sqrt{5}}{2}\right)$ de $\Delta$ estão contidos em $\Delta(a, b)=0$.

Próximo do ponto $p_{+}$segue que:

$\Delta(a, b)=-54675\left[\left(a+\frac{27}{2}\right)^{2}+5\left(b-\frac{5 \sqrt{5}}{2}\right)^{2}+2 \sqrt{5}\left(a+\frac{27}{2}\right)\left(b-\frac{5 \sqrt{5}}{2}\right)\right]+$ h.o.t. 
E obtemos que $p_{ \pm}$são pontos cuspidais de Whitney.

Também a curva $\Delta(a, b)=0$ está contida na região $a \leq-1$ e próximo de $(-1,0)$ esta é dada por $a=-\frac{1}{20} b^{2}+O(3)$. De fato, para $a>-1$ todas as raizes de $\Delta(a, b)$ são complexas.

Pela classificação dos pontos axiumbílicos $E_{3}, E_{4}$ e $E_{5}$ pelo sinal de $\Delta(a, b)$ e de $a$, o diagrama dos pontos axiumbílicos estáveis ( [44], [42] p. 209) é mostrado na Fig. 2.14. 


\section{Capítulo 3}

\section{Pontos Axiumbílicos de Codimensão 1}

No capítulo anterior, fizemos uso do método de Lie-Cartan para descrever uma superfície no fibrado projetivo e sobre esta construímos um campo tangente cuja projeção das curvas integrais definem as linhas axiais ao redor de um ponto axiumbílico. Assim, exibimos as configurações axiais ao redor do axiumbílico, para a qual verificamos três possibilidades: $E_{3}, E_{4}$ e $E_{5}$, cujos sub-índices denotam o número de separatrizes axiumbílicas em cada caso, as quais são projeções das separatrizes de selas das configurações das linhas integrais do campo de Lie-Cartan na vizinhança do eixo projetivo.

O Teorema 2.4 estabelece que estes três casos são os únicos estáveis. A seguir consideramos a questão de como seriam aqueles casos de transição entre estes tipos. Nesta seção vamos descrever os casos de codimensão 1 , os quais denotaremos por $E_{34}^{1}$ e $E_{45}^{1}$. Estes são os únicos casos de axiumbílicos de codimensão 1, e sua configuração será descrita nesta seção. Ambos apresentam uma singularidade do tipo sela-nó, sendo que no caso $E_{34}^{1}$ a variedade central é tangente ao eixo projetivo $p$, enquanto no caso $E_{45}^{1}$ a variedade central é transversal ao eixo $p$.

O caso $E_{34}^{1}$ surge naturalmente da questão sobre a possibilidade de uma raiz dupla do polinômio $R(p)$, o que se caracteriza pela transição entre a existência de duas raizes reais distintas $\left(E_{3}\right)$ e de quatro raizes reais distintas $\left(E_{4}\right)$ de $R(p)$, conforme elucidamos nas figuras da seção anterior. Vale dizer que o ponto $E_{34}^{1}$ é também um ponto axiumbílico transversal. Já o caso $E_{45}^{1}$ ocorre quando violamos a condição de transversalidade do contato das curvas $a_{0}=0$ e $a_{1}=0$ (veja Proposição 1.4). Haverá então a conexão entre um axiumbílico do tipo $E_{4}$ e um do tipo $E_{5}$ os quais colidem configurando o tipo $E_{45}^{1}$ e após a colisão a singularidade desaparece, obtendo apenas linhas regulares na região.

\subsection{O Ponto Axiumbílico $E_{34}^{1}$}

Voltemos à equação (2.2), a qual descreve a superfície de Lie-Cartan ao redor do eixo projetivo. Por meio da equação (2.3), construimos um campo tangente a esta superfície cujas singularidades são dadas pela equação (2.6), que tem como raizes $p_{0}=0$ e as raizes 
de $R(p)$ (equação $(2.7)$ ), cujo discriminante $\Delta(a, b)$ se expressa por

$\Delta(a, b)=16 a^{5}+4\left(b^{2}+68\right) a^{4}+16\left(b^{2}+144\right) a^{3}-8\left(b^{2}-80\right)\left(16+b^{2}\right) a^{2}+96\left(16+b^{2}\right)^{2} a+4\left(16+b^{2}\right)^{3}$.

A Figura 2.14 exibe as regiões do plano $(a, b)$ onde $\Delta>0$ e $\Delta<0$. Estamos aqui interessados na transição $\Delta=0$, e vamos percorrer curva da Figura 2.14 a fim de compreender a configuração local na vizinhança de um ponto umbílico caracterizado por $\Delta=0$.

Vimos no capítulo anterior que:

- $\Delta<0$, então o axiumbílico é do tipo $E_{3}$,

- $\Delta>0, a<0, a \neq-1$, então o axiumbílico é do tipo $E_{4}$,

- $\Delta>0, a>0$, então o axiumbílico é do tipo $E_{5}$.

Na notação deste texto e utilizando a expressão local da superfície na carta de Monge

$$
\left\{\begin{array}{c}
z=R(x, y), \\
w=S(x, y),
\end{array}\right.
$$

onde

$$
\begin{aligned}
R(x, y) & =\frac{r_{20}}{2} x^{2}+r_{11} x y+\frac{r_{02}}{2} y^{2}+\frac{r_{30}}{6} x^{3}+\frac{r_{21}}{2} x^{2} y+\frac{r_{12}}{2} x y^{2}+\frac{r_{03}}{6} y^{3} \\
& +\frac{r_{40}}{24} x^{4}+\frac{r_{31}}{6} x^{3} y+\frac{r_{22}}{4} x^{2} y^{2}+\frac{r_{13}}{6} x y^{3}+\frac{r_{04}}{24} y^{4}+\text { h.o.t. }, \\
S(x, y) & =\frac{s_{20}}{2} x^{2}+s_{11} x y+\frac{s_{02}}{2} y^{2}+\frac{s_{30}}{6} x^{3}+\frac{s_{21}}{2} x^{2} y+\frac{s_{12}}{2} x y^{2}+\frac{s_{03}}{6} y^{3} \\
& +\frac{s_{40}}{24} x^{4}+\frac{s_{31}}{6} x^{3} y+\frac{s_{22}}{4} x^{2} y^{2}+\frac{s_{13}}{6} x y^{3}+\frac{s_{04}}{24} y^{4}+\text { h.o.t.. }
\end{aligned}
$$

Sejam $a$ e $b$ definidos pela Proposição 1.12 .

Definição 3.1. Seja $\alpha: M^{2} \longrightarrow \mathbb{R}^{4}$ imersão $\mathcal{C}^{r}, r \geq 5$ de uma superfície suave, orientada. Um ponto axiumbílico $\mathfrak{p}$ é do tipo $E_{34}^{1}$ se $a$ definido na Proposição 1.12 não se anula e:

i) $\Delta(a, b)=0,(a, b) \neq(-1,0)$ e $(a, b) \neq\left(-\frac{27}{2}, \pm \frac{5}{2} \sqrt{5}\right)$, ou

ii) $b \neq 0$ se $a=-1$.

Observação 3.2. Note que a condição $a \neq 0$ impõe transversalidade às curvas $a_{0}=0 \mathrm{e}$ $a_{1}=0$ em $p$ (Proposição 1.4), a condição $i$ ) requer justamente a existência de uma raiz dupla do polinômio $R(p)$. A condição $i i)$ é necessária porque, como $P(p)=p \cdot R(p)$, no caso em que 0 for raiz dupla de $R$, teremos a existência de uma raiz tripla de $P$.

Proposição 3.3. Suponha $\alpha$ imersão de uma superfície suave, orientada, conexa em $\mathbb{R}^{4}$ de classe $\mathcal{C}^{r}, r \geq 5$ satisfazendo a condição $E_{34}^{1}$ em um ponto axiumbílico $\mathfrak{p}$. Então, as configurações axiais de a na vizinhança de $\mathfrak{p}$ são como na Figura 3.1. 


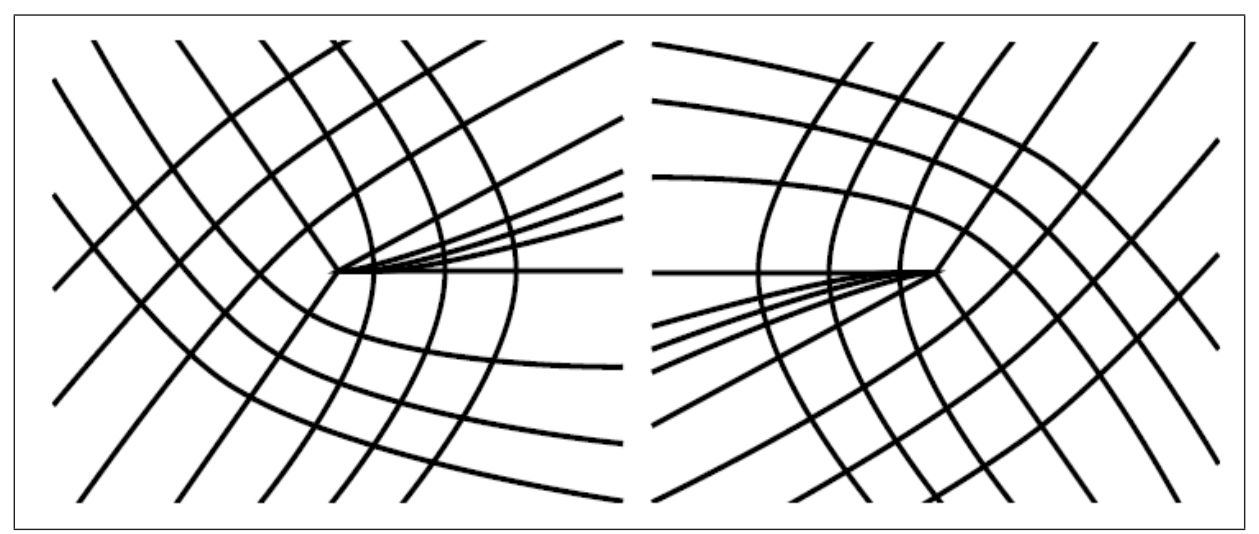

Figura 3.1: Configuração Axial Principal e Configuração Axial Média na vizinhança de um ponto do tipo $E_{34}^{1}$.

Demonstração: Visto que mantemos a condição de transversalidade, então a superfície dada pela equação (2.2) é regular numa vizinhança do eixo projetivo (Afirmação 2.1). Seguimos a mesma análise da seção anterior e temos que as singularidades do campo de Lie-Cartan (equação (2.3)) são dadas pelas raizes da equação (2.6)

$$
P(p)=p \cdot R(p)=p \cdot\left[\left(p^{4}-6 p^{2}+1\right)+\left(1-p^{2}\right)(a+b p)\right]=0
$$

Como já verificamos,

$\Delta(a, b)=16 a^{5}+4\left(b^{2}+68\right) a^{4}+16\left(b^{2}+144\right) a^{3}-8\left(b^{2}-80\right)\left(16+b^{2}\right) a^{2}+96\left(16+b^{2}\right)^{2} a+4\left(16+b^{2}\right)^{3}$, $R( \pm 1)=-4, R(0)=1+a$ e $\lim _{p \longrightarrow \pm \infty} R(p)=+\infty$. Logo, $R$ tem pelo menos duas raizes reais distintas, sendo uma menor que -1 (denotamos por $p_{1}$ ) e outra maior que 1 (denotamos por $p_{4}$ ). Conforme a Definição 3.1, há seis possibilidades no diagrama da Figura 2.14 onde um ponto do tipo $E_{34}^{1}$ pode ocorrer: 


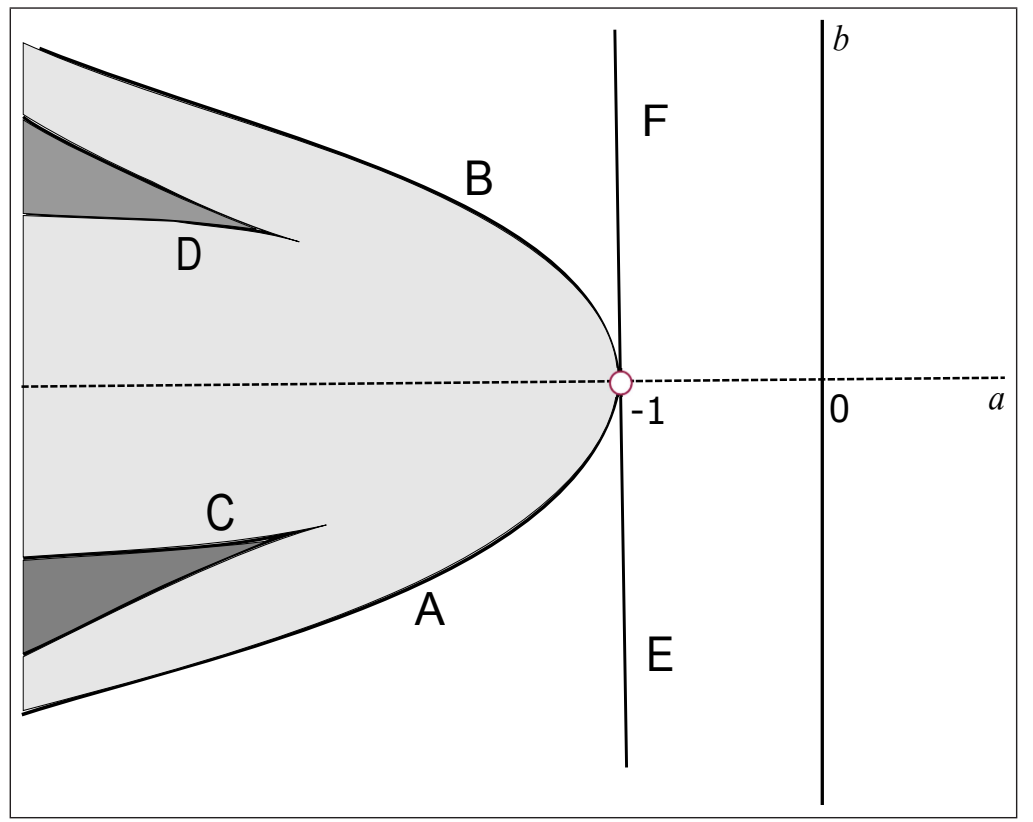

Figura 3.2: Seis Componentes onde ocorre ponto do tipo $E_{34}^{1}$.

As curvas denotadas por $A, B, C, D$ são componentes conexas da curva $\Delta(a, b)=0$ (de onde retiramos o ponto $(-1,0)$, o qual pertence à curva $\Delta(a, b)=0$ ), e portanto estas estão relacionadas à condição $i$ ) da Definição 3.1. As linhas $E$ e $F$, caracterizadas por $i i$ ) da Definição 3.1 tratam da situação em que 0 é raiz dupla do polinômio $P(p)=p \cdot R(p)$. A seguir, ilustramos os casos $A, B, C, D, E, F$.

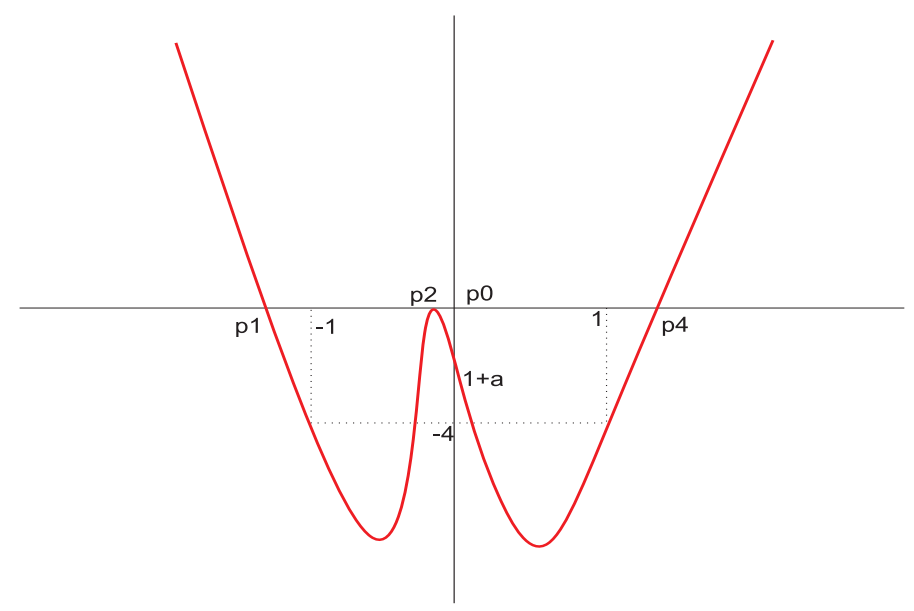

Figura 3.3: Gráfico de $R$ no caso $A$. 


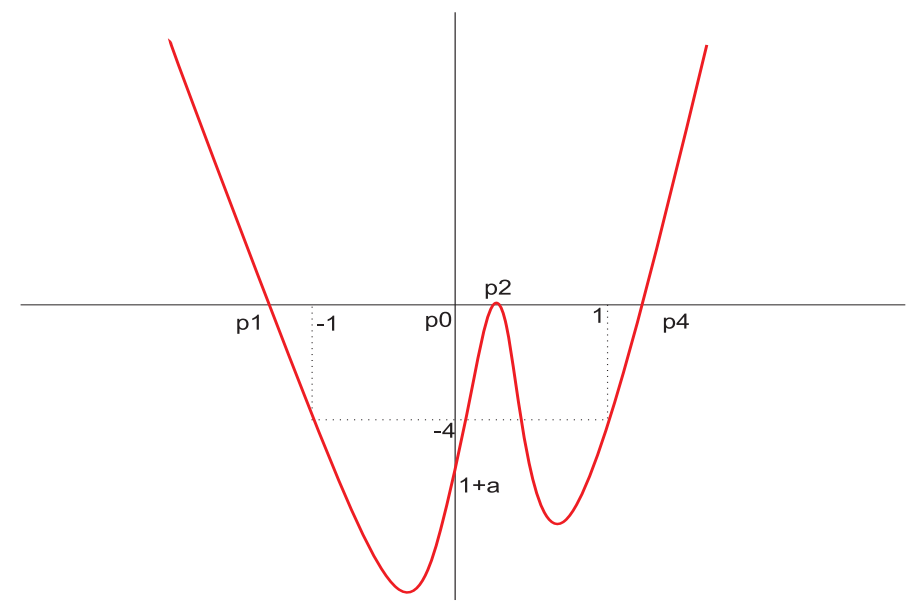

Figura 3.4: Gráfico de $R$ no caso $B$.

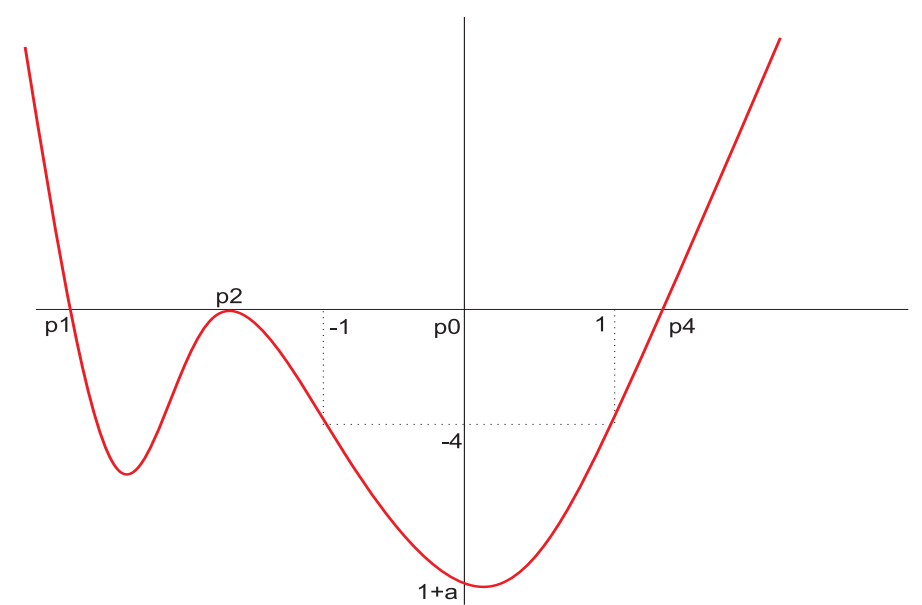

Figura 3.5: Gráfico de $R$ no caso $C$. 


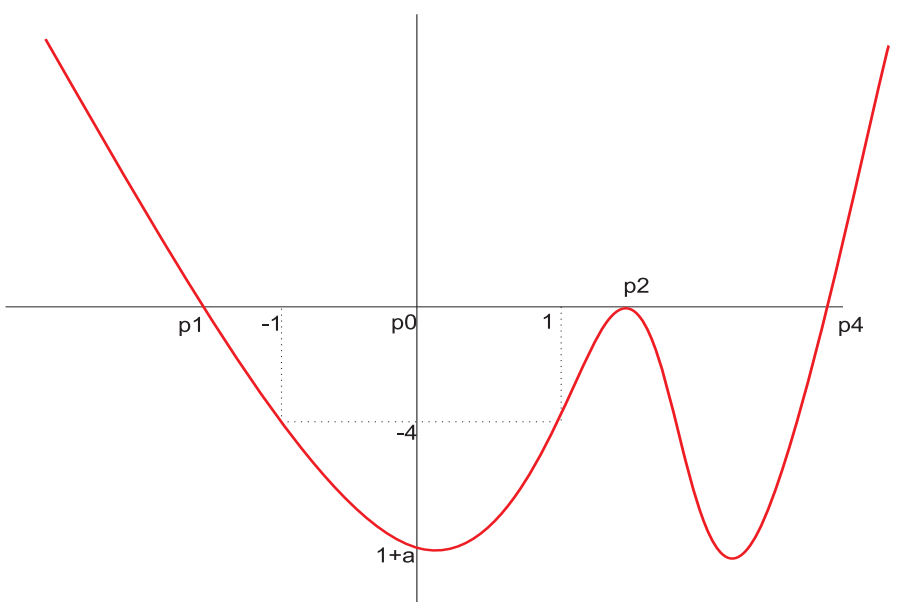

Figura 3.6: Gráfico de $R$ no caso $D$.

Conforme a Equação(2.9) e Equação (2.10), nos pontos singulares $\left(0,0, p_{i}\right)$ a aplicação $D X$ tem por autovalores:

$$
\begin{aligned}
& p_{0}=0 \quad\left\{\begin{array}{l}
\lambda_{1}=a \\
\lambda_{2}=-(a+1)
\end{array}\right. \\
& p_{i} \neq 0 \quad\left\{\begin{array}{l}
\lambda_{1}=\frac{\left(p_{i}^{2}+1\right)^{3}}{\left(p_{i}^{2}-1\right)} \\
\lambda_{2}=-p_{i} R^{\prime}\left(p_{i}\right)
\end{array}\right.
\end{aligned}
$$

sendo que o eixo projetivo está associado ao autovalor $\lambda_{2}$.

Nos casos $A$ e $B$, o quadro dos autovalores referente às singularidades $p_{0}, p_{1}, p_{2}, p_{4}$ fica:

\begin{tabular}{|c|c|c|c|c|}
\hline & $p_{0}$ & $p_{1}$ & $p_{2}$ & $p_{4}$ \\
\hline$\lambda_{1}$ & - & + & - & + \\
\hline$\lambda_{2}$ & + & - & 0 & - \\
\hline & sela & sela & sela-nó & sela \\
\hline
\end{tabular}

Tabela 3.1: Singularidades ao longo do eixo projetivo no caso $E_{34}^{1}$, casos $A$ e $B$.

E nos casos $C$ e $D$ :

\begin{tabular}{|c|c|c|c|c|}
\hline & $p_{0}$ & $p_{1}$ & $p_{2}$ & $p_{4}$ \\
\hline$\lambda_{1}$ & - & + & + & + \\
\hline$\lambda_{2}$ & + & - & 0 & - \\
\hline & sela & sela & sela-nó & sela \\
\hline
\end{tabular}

Tabela 3.2: Singularidades ao longo do eixo projetivo no caso $E_{34}^{1}$, casos $C$ e $D$.

Em todos estes casos, temos uma singularidade do tipo sela-nó e outras 4 singularidades do tipo sela. Se $a=-1$ e $b \neq 0$, então temos que 0 é uma raiz simples do polinômio 
$R$, visto que $R(0)=1+a=0$ mas, como 0 já é raiz de $P(p)=p \cdot R(p)$, resulta que 0 é raiz dupla do polinômio $p$. Ilustramos abaixo tais situações: 


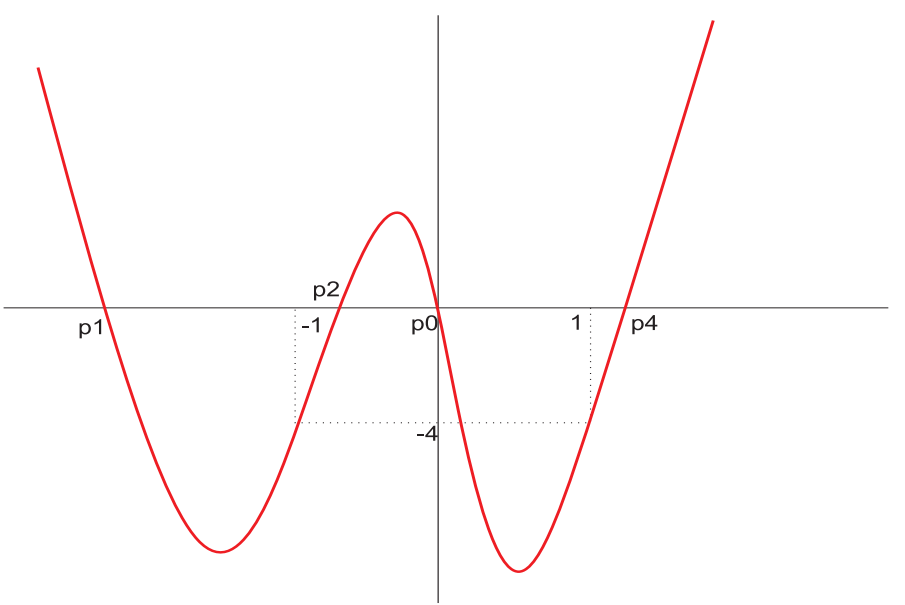

Figura 3.7: Gráfico de $R$ no caso $E$.

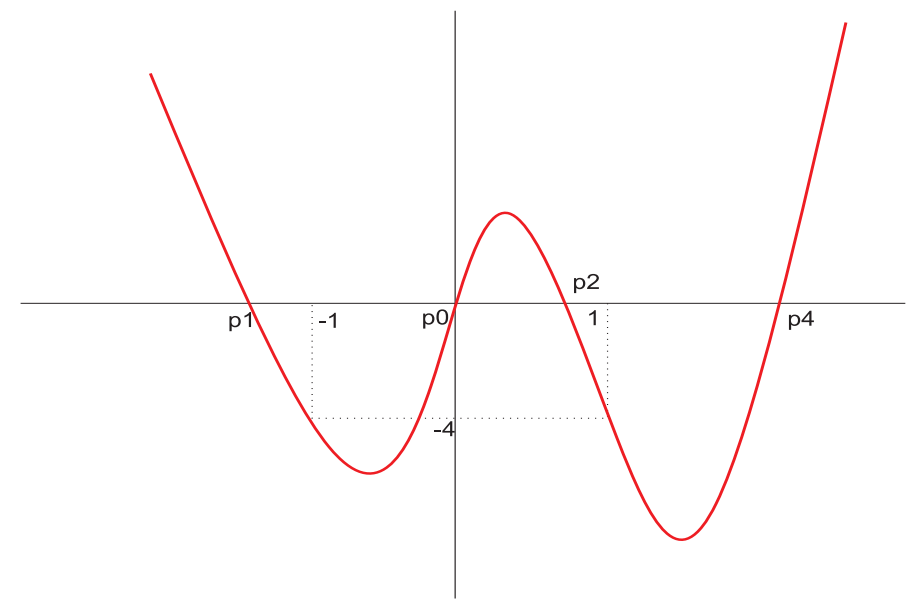

Figura 3.8: Gráfico de $R$ no caso $F$. 
Em tais casos, conforme as equações (2.9) e (2.10), resulta:

\begin{tabular}{|c|c|c|c|c|}
\hline & $p_{0}$ & $p_{1}$ & $p_{2}$ & $p_{4}$ \\
\hline$\lambda_{1}$ & - & + & - & + \\
\hline$\lambda_{2}$ & 0 & - & + & - \\
\hline & sela-nó & sela & sela & sela \\
\hline
\end{tabular}

Tabela 3.3: Singularidades ao longo do eixo projetivo no caso $E_{34}^{1}$, casos $E$ e $F$.

Os seis casos expostos no diagrama, do ponto de vista estrutural todos são os mesmos, por meio de uma rotação de coordenadas no plano $\{x, y\}$. Consideremos o caso exposto no último quadro. Como $\mathcal{G}_{y}(0,0,0)=1$, segue do Teorema das Funções Implícitas que $y(x, p)=x p+O(3)$ está definido na vizinhança de $(0,0,0)$ com $\mathcal{G}(x, y(x, p), p)=0$. Neste caso, o campo de Lie-Cartan restrito à carta $(x, p)$ fica expresso por

$$
\left\{\begin{array}{l}
\dot{x}=-x+b x p+O(2), \\
\dot{p}=\lambda_{2} p-b p^{2}+O(3) .
\end{array}\right.
$$

Como $\lambda_{2}=0$, fica

$$
\left\{\begin{array}{l}
\dot{x}=-x+b x p+O(2), \\
\dot{p}=-b p^{2}+O(3)
\end{array}\right.
$$

com $b \neq 0$. Portanto, $p_{0}=0$ é singularidade do tipo sela-nó com variedade central tangente ao eixo projetivo, e obtemos as configurações na superfície de Lie-Cartan ilustrada na Figura 3.9. 


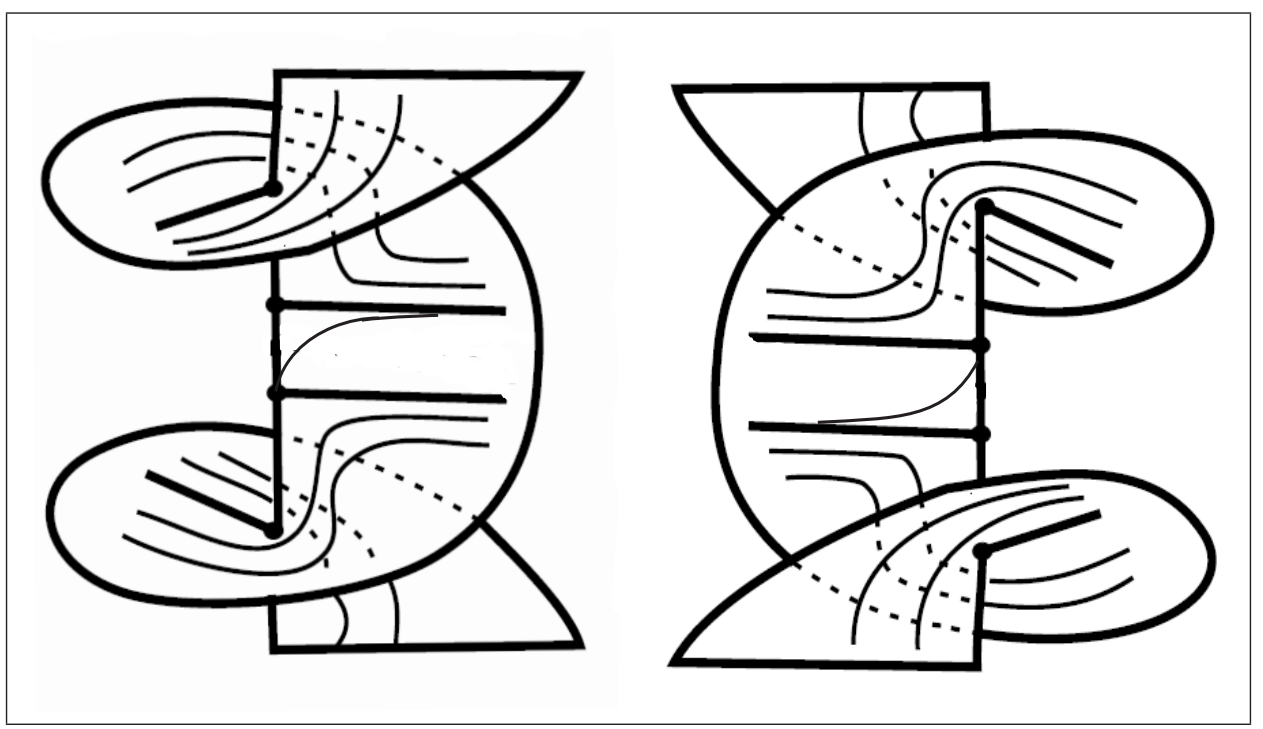

Figura 3.9: Curvas integrais de $\left.X\right|_{\mathcal{G}}=0$ na vizinhança do eixo projetivo no caso $E_{34}^{1}$.

Efetuando a projeção destas linhas, obtemos as configurações ilutradas na Figura 3.10.

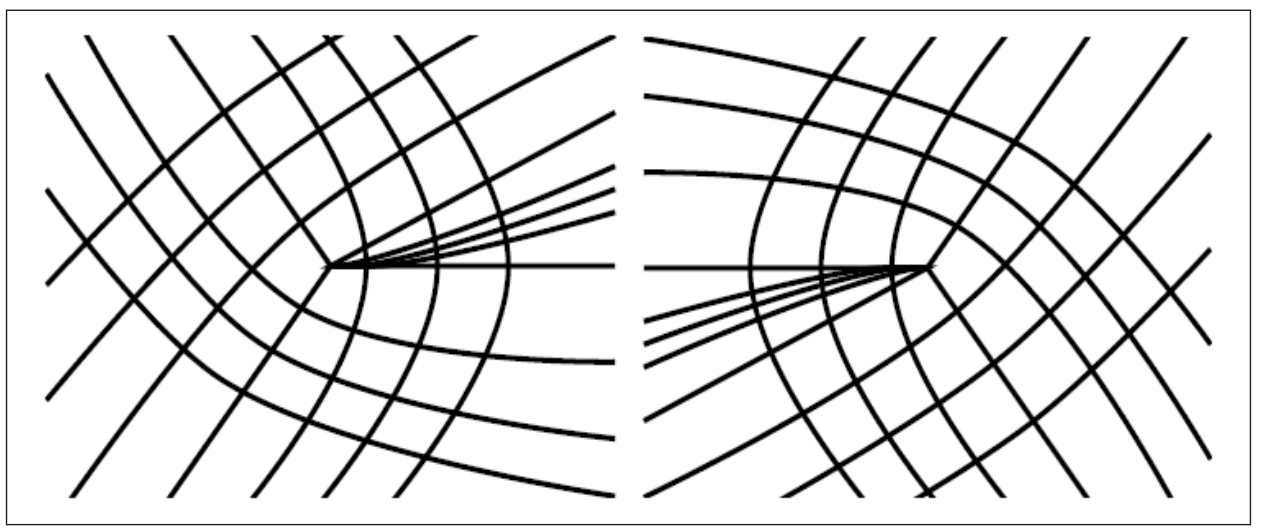

Figura 3.10: Configuração Axial Principal e Configuração Axial Média na vizinhança de um ponto do tipo $E_{34}^{1}$.

Teorema 3.4. Seja $\alpha \in \mathcal{I}^{r}, r \geq 5$ imersão satisfazendo a condição de que o ponto $\mathfrak{p}$ seja axiumbílico do tipo $E_{34}^{1}$. Então, existe uma vizinhança $V$ de $\mathfrak{p}$, uma vizinhança $\mathcal{V}$ de $\alpha$ e uma função $\mathcal{F}: \mathcal{V} \longrightarrow \mathbb{R}$ de classe $\mathcal{C}^{r-3}$ de modo que cada $\mu \in \mathcal{V}$ tem um único ponto axiumbílico $\mathfrak{p}_{\mu} \in V$ sendo:

i) $d \mathcal{F}_{\alpha} \neq 0$

ii) $\mathcal{F}(\mu)<0$ se, e somente se, $\mathfrak{p}_{\mu}$ é axiumbílico do tipo $E_{3}$.

iii) $\mathcal{F}(\mu)>0$ se, e somente se, $\mathfrak{p}_{\mu}$ é axiumbílico do tipo $E_{4}$.

iv) $\mathcal{F}(\mu)=0$ se, e somente se, $\mathfrak{p}_{\mu}$ é axiumbílico do tipo $E_{34}^{1}$. 
Demonstração: Dado que $\mathfrak{p}$ é um ponto axiumbílico transversal de $\alpha$, a existência das vizinhanças $\mathcal{V}$ e $V$ seguem do Teorema das Funções Implícitas. Sendo $\mu \in \mathcal{V}$ imersão com ponto axiumbílico $\mathfrak{p}_{\mu} \in V$, podemos efetuar um movimento rígido $\Gamma_{\mu} \mathrm{em}$ $\mathbb{R}^{4}$ de modo a descrever locamente cada $\mu \in \mathcal{V}$ em termos de uma carta de Monge $\left(x, y, R_{\mu}(x, y), S_{\mu}(x, y)\right)$, com pé em $p_{\mu}$, onde

$$
\begin{aligned}
R_{\mu}(x, y) & =\frac{r_{20}(\mu)}{2} x^{2}+r_{11}(\mu) x y+\frac{r_{02}(\mu)}{2} y^{2}+\frac{r_{30}(\mu)}{6} x^{3}+\frac{r_{21}(\mu)}{2} x^{2} y \\
& +\frac{r_{12}(\mu)}{2} x y^{2}+\frac{r_{03}(\mu)}{6} y^{3}+\text { h.o.t. }, \\
S_{\mu}(x, y) & =\frac{s_{20}(\mu)}{2} x^{2}+s_{11}(\mu) x y+\frac{s_{02}(\mu)}{2} y^{2}+\frac{s_{03}(\mu)}{6} x^{3}+\frac{s_{21}(\mu)}{2} x^{2} y \\
& +\frac{s_{12}(\mu)}{2} x y^{2}+\frac{s_{03}(\mu)}{6} y^{3}+\text { h.o.t. }
\end{aligned}
$$

Para cada $\mu$, podemos efetuar o processo de rotação e homotetia descrito na Seção 1.3, e obtemos $a_{\mu}$ e $b_{\mu}$ em função dos coeficientes da superfície apresentada em uma carta de Monge, conforme feito na Proposição 1.12, sendo os coeficientes em função do parâmetro $\mu \in \mathcal{V}$.

Vamos definir:

$$
\begin{aligned}
\mathcal{F}(\mu) & :=\Delta\left(a_{\mu}, b_{\mu}\right) \\
& =16 a_{\mu}^{5}+4\left(b_{\mu}^{2}+68\right) a_{\mu}^{4}+16\left(b_{\mu}^{2}+144\right) a_{\mu}^{3} \\
& -8\left(b_{\mu}^{2}-80\right)\left(16+b_{\mu}^{2}\right) a_{\mu}^{2}+96\left(16+b_{\mu}^{2}\right)^{2} a_{\mu}+4\left(16+b_{\mu}^{2}\right)^{3},
\end{aligned}
$$

cujas raizes definem localmente a variedade de imersões com um ponto axiumbílico do tipo $E_{34}^{1}$. Note que, devido à particular representação do 3-jato tomada aqui, a condição $a_{\mu}=-1$, a extensão de jato não é transversal, mas tangente, à variedade de jatos com ponto axiumbílico $E_{34}^{1}$. Isto é sempre possível, por uma rotação apropriada do plano $\{x, y\}$ supor que $a_{\mu} \notin\left\{-\frac{27}{2},-1\right\}$.

As afirmações $(i i),(i i i)$ e $(i v)$ decorrem da forma como definimos $\mathcal{F}$ em conjunto com a análise que fizemos na seção anterior, a respeito do sinal do discriminante $\Delta$. Além disso, $d \mathcal{F}_{\alpha} \neq 0$, sendo $\alpha$ imersão com ponto $\mathfrak{p}_{\alpha}$ axiumbílico do tipo $E_{34}^{1}$.

De fato, assumindo $s_{11}(\alpha)=\frac{1}{2} r \neq 0$, segue que:

$$
\begin{aligned}
a_{0}(\mu) & =y+O(2) \\
a_{1}(\mu)(x, y) & =-\frac{4\left(r(\mu)^{2}+s(\mu)^{2}\right) \alpha_{2}(\mu)}{r(\mu)\left(r(\mu) \alpha_{3}(\mu)+s(\mu) \alpha_{4}(\mu)\right)} x+\frac{4\left(s(\mu) \alpha_{3}(\mu)-r(\mu) \alpha_{4}(\mu)\right)}{r(\mu) \alpha_{3}(\mu)+s(\mu) \alpha_{4}(\mu)} y+O(2) \\
= & a(\mu) x+b(\mu) y+O(2), \\
\alpha_{1}=s_{12}-s_{30} & +2 r_{21}, \alpha_{2}=r_{30}-r_{12}+2 s_{21}, \alpha_{3}=s_{03}-s_{21}+2 r_{12}, \alpha_{4}=r_{21}-r_{03}+2 s_{12} .
\end{aligned}
$$


Considere a deformação

$$
\mu=\left(x, y, R_{\alpha}(x, y), S_{\alpha}(x, y)\right)+\left(0,0, t\left(\frac{1}{6} x^{3}-\frac{1}{2} x y^{2}\right), t x^{2} y\right) .
$$

Então, como $\alpha_{2}=r_{30}-r_{12}+2 s_{21}$, segue que $a(\mu)=-\frac{4\left(r^{2}+s^{2}\right)\left(\alpha_{2}+t\right)}{r\left(r \alpha_{3}+s \alpha_{4}\right)}$ e

$$
\frac{d}{d t}\left(\left.\Delta(a(\mu), b(\mu))\right|_{t=0}=\frac{\partial \Delta}{\partial a} \cdot \frac{d a}{d t}=\frac{\partial \Delta}{\partial a} \cdot\left(-\frac{4\left(r^{2}+s^{2}\right)}{r\left(r \alpha_{3}+s \alpha_{4}\right)}\right) \neq 0 .\right.
$$

Quando $s_{11}(\alpha)=0$ segue que $r_{11}(\alpha)=-\frac{1}{2} s \neq 0, \alpha_{1} \alpha_{4} \neq 0$ and $\alpha_{2}(\mu)=0$. Então, considere a deformação

$$
\mu=\left(x, y, R_{\alpha}(x, y), S_{\alpha}(x, y)\right)+\left(0,0, t x^{2} y, t\left(-\frac{1}{6} x^{3}+\frac{1}{2} x y^{2}\right)\right)
$$

Então, $a(\mu)=\frac{4\left(\alpha_{1}+t\right)}{\alpha_{4}} \mathrm{e}$

$$
\frac{d}{d t}\left(\left.\Delta(a(\mu), b(\mu))\right|_{t=0}=\frac{\partial \Delta}{\partial a} \cdot \frac{d a}{d t}=\frac{\partial \Delta}{\partial a} \cdot\left(\frac{4}{\alpha_{4}}\right) \neq 0 .\right.
$$

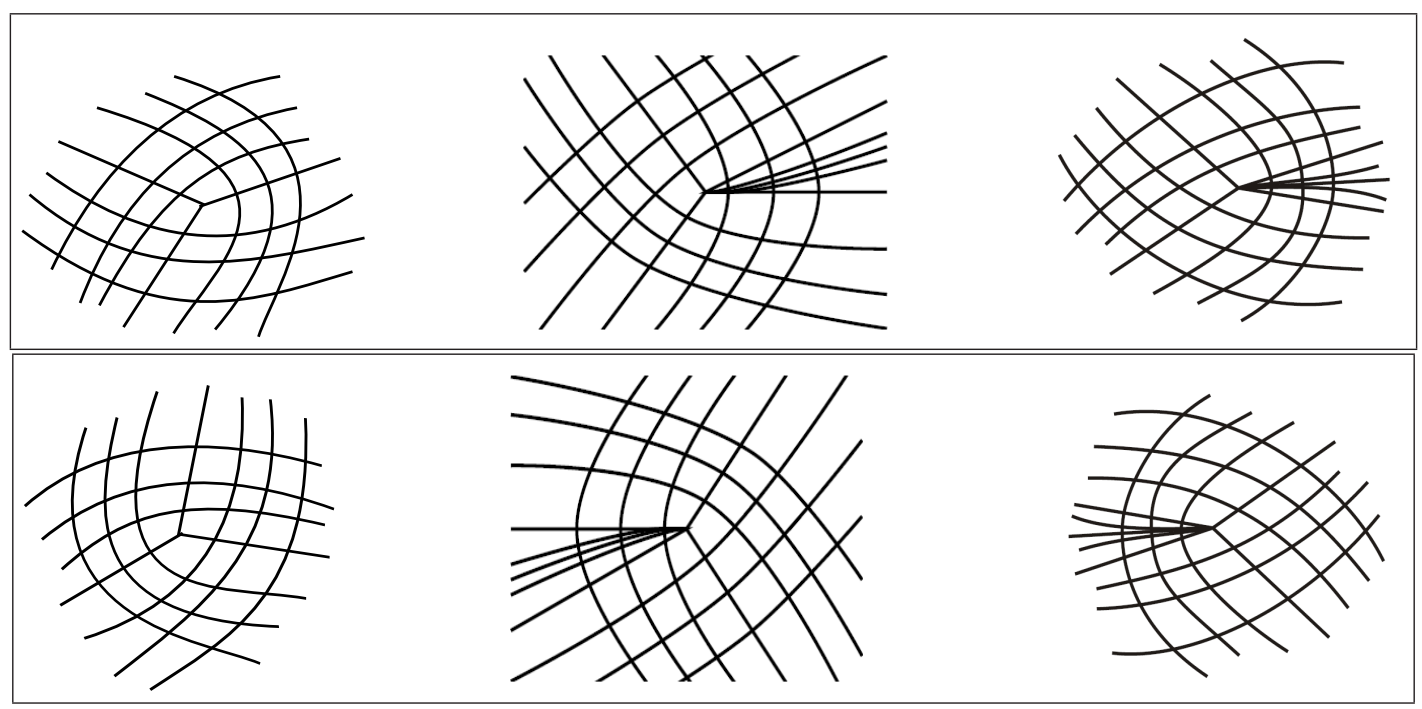

Figura 3.11: Configurações Axiais na vizinhança de pontos axiumbílicos. $E_{3}$ (esquerda), $E_{34}^{1}$ (centro) and $E_{4}$ (direita). 


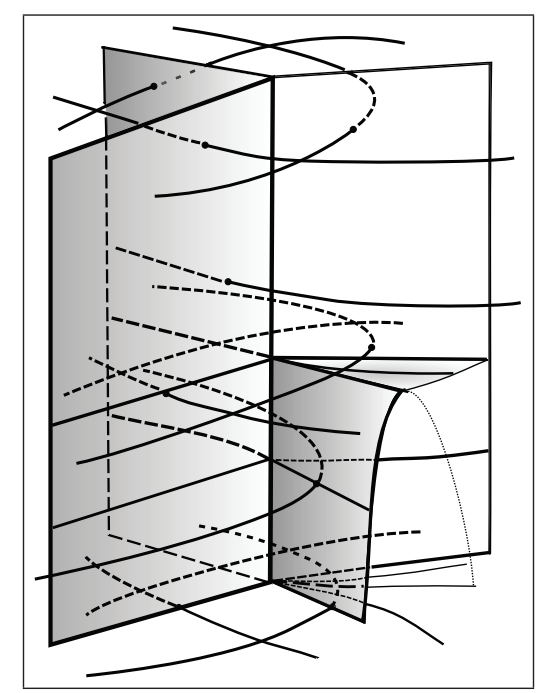

Figura 3.12: Diagrama de Bifurcação das Configurações Axiais próximas de um ponto axiumbílico $E_{34}^{1}$ e a estrutura de separatrizes.

\subsection{O ponto Axiumbílico $E_{45}^{1}$}

Considere uma carta de Monge conforme as equações (1.11) and (1.12). Suponha que a origem seja um ponto axiumbílico, o qual é expresso por:

$$
\begin{aligned}
R(x, y) & =\frac{r_{20}}{2} x^{2}+r_{11} x y+\frac{r_{02}}{2} y^{2}+\frac{r_{30}}{6} x^{3}+\frac{r_{21}}{2} x^{2} y+\frac{r_{12}}{2} x y^{2}+\frac{r_{03}}{6} y^{3} \\
& +\frac{r_{40}}{24} x^{4}+\frac{r_{31}}{6} x^{3} y+\frac{r_{22}}{4} x^{2} y^{2}+\frac{r_{13}}{6} x y^{3}+\frac{r_{04}}{24} y^{4}+\text { h.o.t. }, \\
S(x, y) & =\frac{s_{20}}{2} x^{2}+s_{11} x y+\frac{s_{02}}{2} y^{2}+\frac{s_{30}}{6} x^{3}+\frac{s_{21}}{2} x^{2} y+\frac{s_{12}}{2} x y^{2}+\frac{s_{03}}{6} y^{3} \\
& +\frac{s_{40}}{24} x^{4}+\frac{s_{31}}{6} x^{3} y+\frac{s_{22}}{4} x^{2} y^{2}+\frac{s_{13}}{6} x y^{3}+\frac{s_{04}}{24} y^{4}+\text { h.o.t. },
\end{aligned}
$$

com $r_{02}=r_{20}+r, r_{11}=-\frac{1}{2} s, s_{02}=s_{20}+s, s_{11}=\frac{1}{2} r$.

Seja

$$
\begin{aligned}
& \alpha_{1}=s_{12}-s_{30}+2 r_{21}, \alpha_{2}=r_{30}-r_{12}+2 s_{21}, \\
& \alpha_{3}=s_{03}-s_{21}+2 r_{12}, \alpha_{4}=r_{21}-r_{03}+2 s_{12}, \\
& \beta_{1}=s_{22}-s_{40}+2 r_{31}, \beta_{2}=r_{40}-r_{22}+2 s_{31}, \\
& \beta_{3}=s_{13}-s_{31}+2 r_{22}, \beta_{4}=r_{31}-r_{13}+2 s_{22}, \\
& \beta_{5}=s_{04}-s_{22}+2 r_{13}, \beta_{6}=r_{22}-r_{04}+2 s_{13} .
\end{aligned}
$$

As funções $a_{0}$ and $a_{1}$ (Proposição 1.4) são dadas por

$$
a_{0}(x, y)=a_{10} x+a_{01} y+\frac{1}{2} a_{20} x^{2}+a_{11} x y+\frac{1}{2} a_{02} y^{2}+\text { h.o.t. }
$$

$\mathrm{e}$

$$
a_{1}(x, y)=b_{10} x+b_{01} y+\frac{1}{2} b_{20} x^{2}+b_{11} x y+\frac{1}{2} b_{02} y^{2}+\text { h.o.t. }
$$


onde

$$
\begin{aligned}
a_{10}= & \frac{1}{2}\left(r \alpha_{1}+s \alpha_{2}\right), \quad a_{01}=\frac{1}{2}\left(r \alpha_{3}+s \alpha_{4}\right), \\
a_{20}= & -\alpha_{2} r_{21}+\alpha_{1} s_{21}+\left[\frac{\beta_{1}}{4}+\frac{s_{20}}{2}\left(r_{20}^{2}+s_{20}^{2}\right)\right] r+\left[\frac{\beta_{2}}{4}-\frac{r_{20}}{2}\left(r_{20}^{2}+s_{20}^{2}\right)\right] s \\
& +\left(r_{20}^{2}-s_{20}^{2}\right) s r-\frac{3}{8}\left(r^{2}+s^{2}\right)\left(s_{20} r-r_{20} s\right)+r_{20} s_{20}\left(s^{2}-r^{2}\right), \\
& a_{11}=-r_{12} \alpha_{2}+s_{12} \alpha_{1}-r_{21} \alpha_{4}+s_{21} \alpha_{3}-\left[\frac{\beta_{3}}{2}+r_{20}\left(r_{20}^{2}+s_{20}^{2}\right)\right] r \\
& +\left[\frac{\beta_{4}}{2}-s_{20}\left(r_{20}^{2}+s_{20}^{2}\right)\right] s-2 s_{20} r_{20} r s-\frac{1}{2}\left(3 s_{20}^{2}+r_{20}^{2}\right) s^{2} \\
& -\frac{1}{2}\left(3 r_{20}^{2}+s_{20}^{2}\right) r^{2}-\frac{3}{8}\left(r^{2}+s^{2}\right)^{2}-\frac{5}{4}\left(r^{2}+s^{2}\right)\left(r_{20} r+s_{20} s\right), \\
& a_{02}=-r_{12} \alpha_{4}+s_{12} \alpha_{3}+\left[\frac{\beta_{5}}{2}-\frac{s_{20}}{2}\left(r_{20}^{2}+s_{20}^{2}\right)\right] r+\left[\frac{\beta_{6}}{2}+\frac{r_{20}}{2}\left(r_{20}^{2}+s_{20}^{2}\right)\right] s \\
& +\left(-2 s_{20}^{2}+2 r_{20}^{2}\right) s r+2 s_{20} r_{20}\left(s^{2}-2 r^{2}\right)+-\frac{9}{8}\left(r^{2}+s^{2}\right)\left(r s_{20}-s r_{20}\right), \\
b_{02}=\alpha_{3}^{2}+ & \alpha_{4}^{2}+4\left(r_{12}^{2}+s_{12}^{2}\right)+4 s_{12}\left(r_{21}-r_{03}\right)+4 r_{12}\left(s_{03}-s_{21}\right) \\
+ & \left.-\beta_{6}-2 r_{20}\left(r_{20}^{2}+s_{20}^{2}\right)\right] r+\left[\beta_{5}-2 s_{20}\left(r_{20}^{2}+s_{20}^{2}\right)\right] s+2\left(r_{20}^{2}-3 s_{20}^{2}\right) s^{2}+2\left(s_{20}^{2}-r_{20}^{2}\right) r^{2} . \\
b_{10}=2\left(s \alpha_{1}-r \alpha_{2}\right), & b_{01}=2\left(s \alpha_{3}-r \alpha_{4}\right), \\
b_{20}= & \alpha_{1}^{2}+\alpha_{2}^{2}-4\left(s_{21} \alpha_{2}+r_{21} \alpha_{1}\right)+\left[-\beta_{2}+2 r_{20}\left(r_{20}^{2}+s_{20}^{2}\right)\right] r \\
+ & \left.\left.+\beta_{1}+\alpha_{2} \alpha_{4}\right)-4\left(\alpha_{1} r_{12}+\alpha_{2} s_{12}+\alpha_{3} r_{21}+\alpha_{4} s_{21}\right)+2\left[s_{20}^{2}\right)\right] s-\frac{1}{2}\left(r^{2}+s_{20}^{2}\right)\left(s_{20} s+r_{20} r\right)+4\left(r_{20} s-s_{20} r\right)^{2}, \\
& \left.\left.+s_{20}^{2}\right)\right] r
\end{aligned}
$$

Definição 3.5. Um ponto axiumbílico é do tipo $E_{45}^{1}$ se a variedade $\mathbb{L}_{\alpha}$ possui exatamente 4 pontos singulares os quais são do tipo Morse ao longo do eixo projetivo.

Proposição 3.6. Considere uma carta de Monge e uma homotetia de modo que a equação diferencial das linhas axiais seja escrita da forma:

$$
a_{0}(x, y)\left(d x^{4}-6 d x^{2} d y^{2}+d y^{4}\right)+a_{1}(x, y) d x d y\left(d x^{2}-d y^{2}\right)+O(3)=0
$$

sendo

$$
\begin{aligned}
& a_{0}(x, y)=y+\frac{1}{2} a_{20} x^{2}+a_{11} x y+\frac{1}{2} a_{02} y^{2}+\text { h.o.t. } \\
& a_{1}(x, y)=b_{01} y+\frac{1}{2} b_{20} x^{2}+b_{11} x y+\frac{1}{2} b_{02} y^{2}+\text { h.o.t. }
\end{aligned}
$$


Então, as seguintes condições são equivalentes:

i) as curvas $a_{0}=0$ e $a_{1}=0$ são regulares e tem contato quadrático em 0 ,

ii) o ponto axiumbílico 0 é do tipo $E_{45}^{1}$, segundo a Definição 3.5,

iii) O campo de Lie-Cartan definido sobre $\mathbb{L}_{\alpha}$ tem uma sela-nó quadrática sobre o eixo projetivo cuja variedade central é transversal à linha projetiva.

Demonstração: A equação diferencial das linhas axiais pode ser escrita como

$$
a_{0}(x, y)\left(d x^{4}-6 d x^{2} d y^{2}+d y^{4}\right)+a_{1}(x, y) d x d y\left(d x^{2}-d y^{2}\right)+O(3)=0,
$$

onde

$$
\begin{aligned}
& a_{0}(x, y)=a_{10} x+a_{01} y+\frac{1}{2} a_{20} x^{2}+a_{11} x y+\frac{1}{2} a_{02} y^{2}+\text { h.o.t. } \\
& a_{1}(x, y)=b_{10} x+b_{01} y+\frac{1}{2} b_{20} x^{2}+b_{11} x y+\frac{1}{2} b_{02} y^{2}+\text { h.o.t.. }
\end{aligned}
$$

Os coeficientes $a_{i j}$ e $b_{i j}, i, j=0,1,2$ são dados pelas equações (3.6) e (3.7). Aqui $O(3)$ denota os termos de ordem maior ou igual a 3 nas variáveis $x$ e $y$.

No que se segue, vamos considerar uma carta de Monge de modo que $a_{10}=0$. Isto é possível conforme mostrado no Lema 1.11 e Proposição 1.12. Como o contato entre $a_{0}=0$ e $a_{1}=0$ é suposto ser quadrático, disso resulta que $b_{10}=0$ e $a_{01} \cdot b_{01} \neq 0$. Também por uma homotetia é possível obter $a_{01}=1$.

Temos assim

$$
\begin{aligned}
& a_{0}(x, y)=y+\frac{1}{2} a_{20} x^{2}+a_{11} x y+\frac{1}{2} a_{02} y^{2}+h . o . t . \\
& a_{1}(x, y)=b_{01} y+\frac{1}{2} b_{20} x^{2}+b_{11} x y+\frac{1}{2} b_{02} y^{2}+\text { h.o.t. }
\end{aligned}
$$

Além disso, a condição de contato quadrático entre as duas curvas regulares é expressa por:

$$
\chi=b_{20}-a_{20} b_{01} \neq 0 .
$$

Afirmação 3.7. Na vizinhança de $(0,0,0)$, o campo de Lie-Cartan restrito à superfície $\mathcal{G}=0$, pode ser expresso na carta $(x, p)$ por

$$
\left\{\begin{array}{l}
\dot{x}=\frac{\chi}{2} x^{2}+O(3) \\
\dot{p}=-p+\frac{3}{2} a_{11} a_{20} x^{2}-\left(a_{11}+\chi\right) p-b_{01} p^{2}+O(3)
\end{array}\right.
$$

e $(0,0,0)$ é do tipo sela-nó quando $\chi \neq 0$.

Prova: Como $\mathcal{G}_{y}(0,0,0)=1 \neq 0$, segue do Teorema da Função Implícita que localmente $y=y(x, p)$ e $\mathcal{G}(x, y(x, p), p)=0$. 
A expansão de Taylor de $y(x, p)$ na vizinhança de $(x, p)=(0,0)$ é dada por

$$
y(x, p)=-\frac{1}{2} a_{20} x^{2}+O(3) .
$$

O campo de Lie-Cartan restrito à superfície $\mathcal{G}=0$ é dado por

$$
\left\{\begin{array}{l}
\dot{x}=\mathcal{G}_{p}(x, y(x, p), p)=\frac{1}{2} \chi x^{2}+O(3), \\
\dot{p}=-\left(\mathcal{G}_{x}+p \mathcal{G}_{y}\right)(x, y(x, p), p)=-p+\frac{3}{2} a_{11} a_{20} x^{2}-\left(\chi+a_{11}\right) p-b_{01} p^{2}+O(3)
\end{array}\right.
$$

Os autovalores do campo (3.10) em 0 são $\lambda_{1}=0$ e $\lambda_{2}=-1$ com respectivos autoespaços associados $\ell_{1}=\left(1,-a_{20}\right)$ and $\ell_{2}=(0,1)$. Pela Teoria de Variedades Invariantes a varidadade central é tangente a $\ell_{1}$ e é dada por

$$
W^{c}=\left\{\left(x,-a_{20} x+\frac{3}{2} a_{20}\left(\chi+a_{11}\right) x^{2}+O(3)\right)\right\} .
$$

A restrição do campo (3.10) à variedade central é dada por

$$
\left[\frac{1}{2} \chi x^{2}+0(3)\right] \frac{\partial}{\partial x}
$$

Afirmação 3.8. A função $\mathcal{G}$ tem exatamente 4 pontos críticos na linha projetiva, e estes são de tipo Morse de índice 1 ou 2 se, e somente se, $\chi \neq 0$.

Prova: Os pontos críticos de $\mathcal{G}$ ao longo do eixo projetivo são determinados por

$$
S(p)=\mathcal{G}_{v}(0,0, p)=\left(p^{4}-6 p^{2}+1\right)+b_{01} p\left(1-p^{2}\right)=0,
$$

a qual tem 4 raizes reais simples localizadas em $(-\infty,-1) \cap(-1,0) \cap(0,1) \cap(1, \infty)$. Isto segue de $S( \pm 1)=-4, S(0)=1$ e do discriminante:

$$
\Delta(S)=4\left(16+b_{01}^{2}\right)^{3}>0
$$

Ao longo do eixo projetivo, o determinante da Hessiana de $\mathcal{G}$ é:

$$
\operatorname{Hess} \mathcal{G}(0,0, p)=-\left(a_{20}\left(1-6 p^{2}+p^{4}\right)+b_{20} p\left(1-p^{2}\right)\right)\left(b_{01}-12 p-3 b_{01} p^{2}+4 p^{3}\right)^{2}
$$

O resultante entre $S(p)$ e $\operatorname{Hess} \mathcal{G}(0,0, p)$ é

$$
256 \chi^{4}\left(16+b_{01}^{2}\right)^{6}
$$

e portanto $\operatorname{Hess} \mathcal{G}(0,0, p) \neq 0$ nos pontos críticos de $\mathcal{G}$. Portanto, estes são do tipo Morse. 
Como $\mathcal{G}(0,0, p)=0$ então o índice dos pontos críticos é 1 ou 2 e então localmente o conjunto $\mathcal{G}=0$ é um cone.

Os autovalores da derivada do campo de Lie-Cartan nos pontos $(0,0, p)$ são

$$
\begin{aligned}
& \lambda_{1}=-p\left(-4 p^{3}+3 b_{01} p^{2}+12 p-b_{01}\right), \\
& \lambda_{2}=-1+18 p^{2}-5 p^{4}-2 b_{01} p+4 b_{01} p^{3} .
\end{aligned}
$$

Nos pontos críticos $p_{i}$ (satisfazendo $S\left(p_{i}\right)=0$ ) segue que

$$
\lambda_{1}=-\lambda_{2}=\frac{p^{6}+3 p^{4}+3 p^{2}+1}{p^{2}-1}
$$

e então

$$
\lambda_{1}^{i} \lambda_{2}^{i}<0, \text { para } i=1, \ldots, 4
$$

Portanto, estes 4 pontos são selas do Campo de Lie-Cartan. Como a linha projetiva é invariante, segue que a outra variedade invariante (estável ou instável) é transversal ao eixo projetivo.

Sobre um ponto axiumbílico $p$ temos então 5 equilíbrios do campo de Lie-Cartan, $p_{0}, p_{1}, p_{2}, p_{3}, p_{4}$, sendo $p_{0}=0$ do tipo sela-nó com variedade central transversal ao eixo projetivo e as demais singularidades do tipo cone de Morse na variedade $\mathbb{L}_{\alpha}$ sobre o ponto axiumbílico. A Figura 3.13 ilustra a posição desses pontos tipo Morse (note que as retas $p=0, p=-1, p=1$ são assíntotas horizontais).

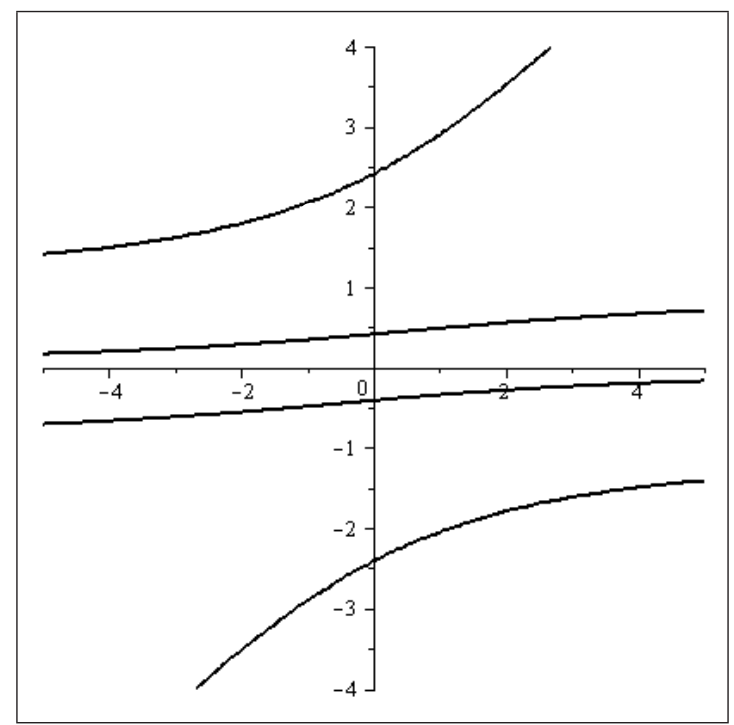

Figura 3.13: As 4 singularidades $p_{i}$. 
Neste caso a superfície de Lie-Cartan $\mathbb{L}_{\alpha}$ é constituída de 4 folhas no fibrado projetivo, as quais têm contato cônico nos 4 pontos críticos expressos por $p_{1}, p_{2}, p_{3}, p_{4}$, conforme ilustramos na Figura 3.14. Destacamos na Figura 3.15 as linhas integrais do Campo de Lie-Cartan na variedade cônica na vizinhança dos pontos em questão.

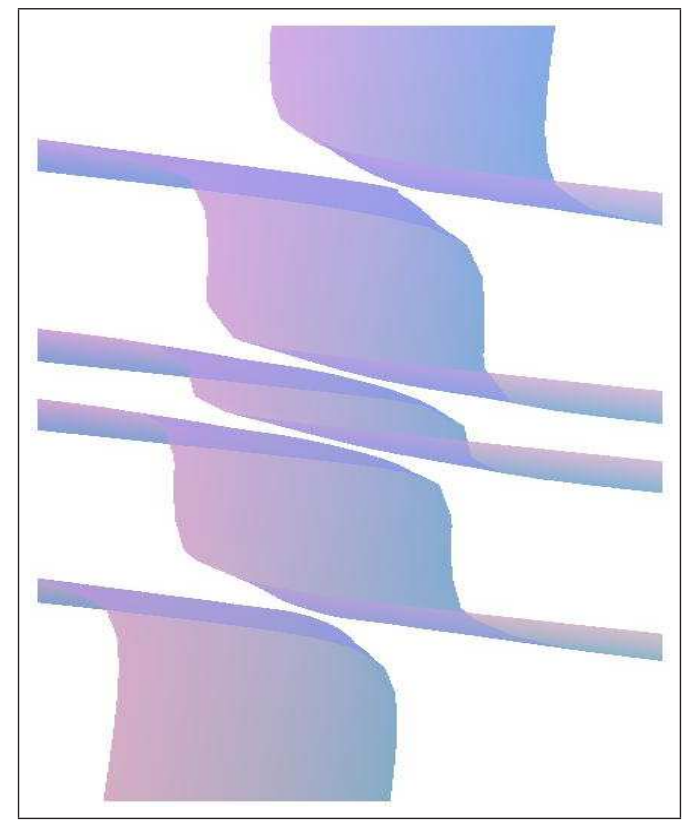

Figura 3.14: Folhas da superfície de Lie-Cartan. O eixo $p$ está inclinado para favorecer a visualização. 


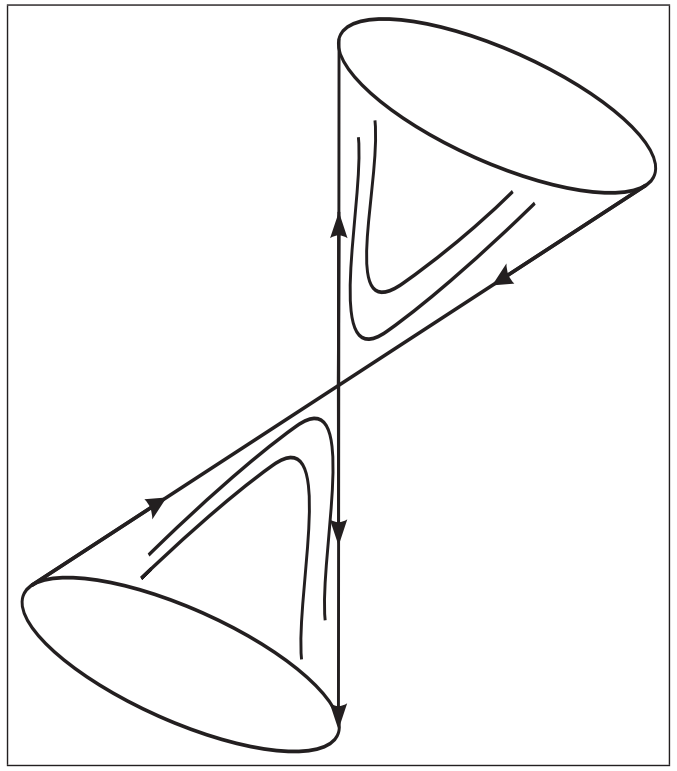

Figura 3.15: Ilustração da superfície com os pontos críticos tipo Morse e retrato de fase do campo de Lie-Cartan.

Proposição 3.9. Seja $\alpha \in \mathcal{I}^{r}, r \geq 5$ e p um ponto axiumbílico. Suponha, na carta de Monge expressa pelas equações (3.2) e (3.3), que $\alpha_{1}=\alpha_{3}=0$ e $\chi \neq 0$. Então p é um ponto axiumbilico do tipo $E_{45}^{1}$ e a configuração axial de a numa vizinhança de $\mathfrak{p}$ é como na Figura 3.16.

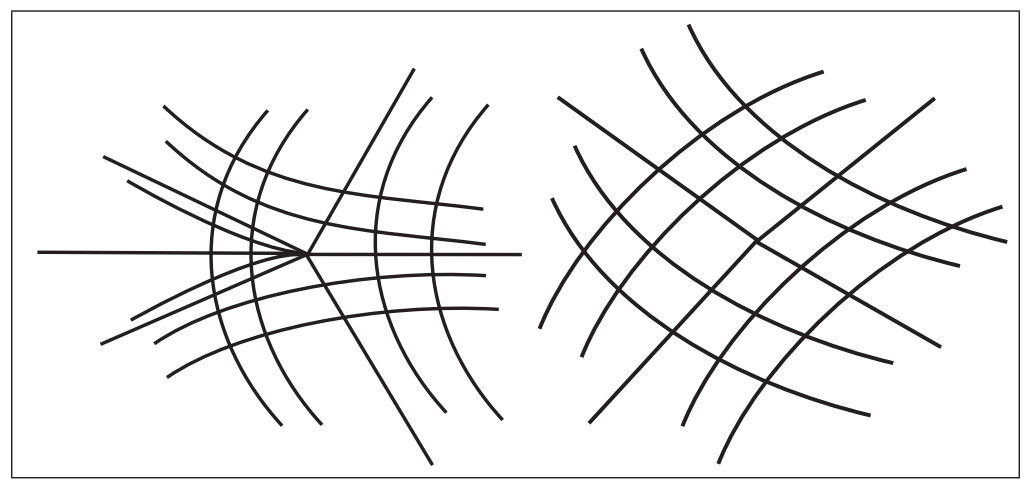

Figura 3.16: Configurações Axiais na vizinhança de um ponto axiumbílico do tipo $E_{4,5}^{1}$.

Demonstração: A condição $\alpha_{1}=\alpha_{3}=0$ implica no contato não-transversal das curvas $a_{0}=0$ and $a_{1}=0$ no ponto axiumbílico $\mathfrak{p}$ expresso na carta de Monge por $(0,0)$. Pelo Lema 1.11 e Proposição 1.12, e possível expressar estas curvas como na equação (3.8). Assumindo $\chi \neq 0$, temos contato quadrático das curvas no ponto axiumbílico.

Proposição 3.6 nos garante que sobre o ponto axiumbílico temos cinco equilíbrios do campo de Lie-Cartan, sendo que em um deles a superfície é regular e o qual é do tipo sela-nó com variedade central transversal ao eixo $p$. (Afirmação 3.7).

Os demais equilíbrios são pontos críticos do tipo Morse da superfície de Lie-Cartan. Na vizinhança destes pontos, os níveis $\mathcal{G}=0$ são localmente cones, e os 4 pontos são selas 


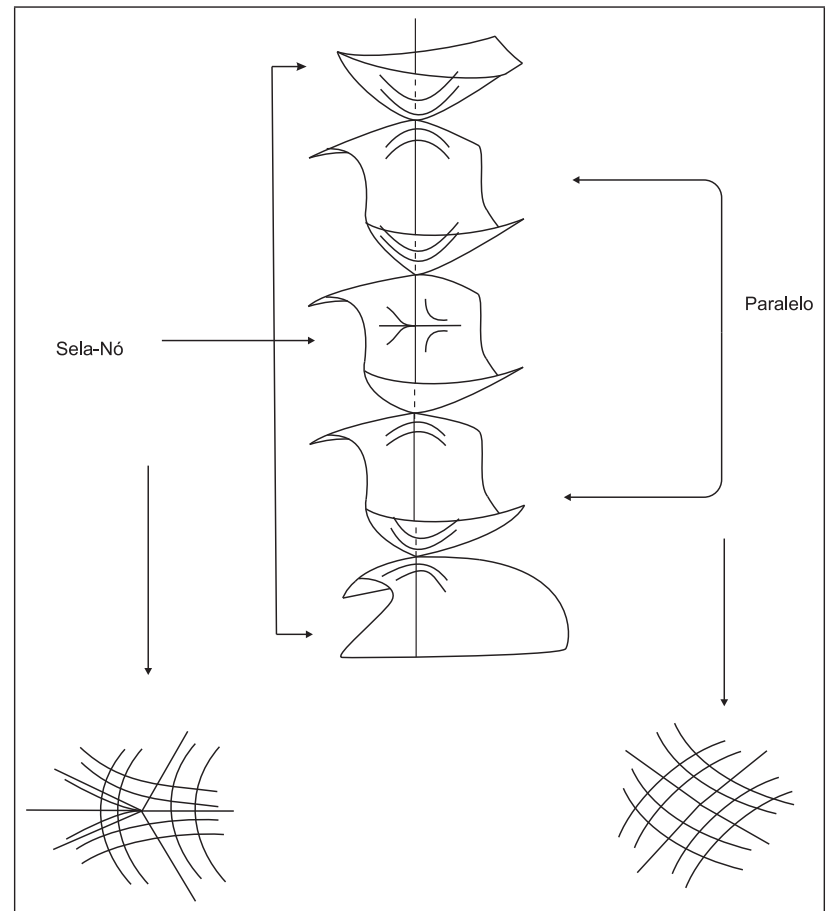

Figura 3.17: Campo de Lie-Cartan próximo de um ponto axiumbílico $E_{45}^{1}$ e a configuração axial (principal e média).

do Campo de Lie-Cartan (Afirmação 3.8).

Enfim, concluimos a configuração descrita na Figura 3.17, cuja projeção dos setores paralelo e sela-nó descrevem a configuração axial principal e configuração axial média próximas ao ponto axiumbílico $\mathfrak{p}$ de tipo $E_{45}^{1}$ (Figura 3.16).

Teorema 3.10. Seja $\alpha \in \mathcal{I}^{r}, r \geq 5$, imersão satisfazendo a condição $E_{45}^{1}$ em um ponto axiumbílico $\mathfrak{p}$. Então existe uma vizinhança $V$ de $\mathfrak{p}$, uma vizinhança $\mathcal{V}$ de $\alpha$ e uma função

$$
F: \mathcal{V} \longrightarrow \mathbb{R}
$$

de classe $\mathcal{C}^{r-3}$ tal que:

i) $d F_{\alpha} \neq 0$

ii) $F(\mu)=0$ se, e somente se, $\mu \in \mathcal{V}$ tem um único ponto axiumbílico em $V$, o qual é do tipo $E_{45}^{1}$,

iii) $F(\mu)<0$ se, e somente se, $\mu$ tem exatamente dois pontos axiumbílicos em $V$, sendo um do tipo $E_{4}$ e o outro do tipo $E_{5}$,

iv) $F(\mu)>0$ se, e somente se, $\mu$ não tem pontos axiumbilicos em $V$.

Demonstração: Pela Proposição 3.6, como $\alpha$ é uma imersão com ponto axiumbílico p do tipo $E_{45}^{1}$, as curvas $a_{0}^{\alpha}=0$ e $a_{1}^{\alpha}=0$ tem contato quadrático em $\mathfrak{p}$. 
Como

$$
\frac{\partial a_{0}^{\alpha}}{\partial y}(0,0)=a_{01} \neq 0,
$$

segue do Teorema das Funções Implícitas que, localmente, para $\mu$ em uma vizinhança $\mathcal{V}$ de $\alpha, y=y_{\mu}(x)$ e $a_{0}^{\mu}\left(x, y_{\mu}(x)\right)=0$.

Além disso,

$$
\frac{\partial^{2} a_{1}^{\alpha}}{\partial x^{2}}(0,0)=b_{20} \neq 0,
$$

e então $x=x_{\mu}$ é uma solução local de

$$
\frac{\partial a_{1}^{\mu}}{\partial x}\left(x_{\mu}, y_{\mu}\left(x_{\mu}\right)\right)=0 .
$$

Defina $\mathcal{F}(\mu)=a_{1}^{\mu}\left(x_{\mu}, y_{\mu}\left(x_{\mu}\right)\right)$ e considere a perturbação

$$
h_{t}(x, y)=(x, y, R(x, y)+t x y, S(x, y)+t x y) .
$$

Segue que

$$
\left.\frac{d F(t)}{d t}\right|_{t=0} \neq 0
$$

e então $d F_{\alpha} \neq 0$.

O ponto axiumbílico $E_{45}^{1}$ é portanto a transição entre zero e dois pontos axiumbílicos, um do tipo $E_{4}$ e outro do tipo $E_{5}$.

Nas figuras 3.18 e 3.20 ilustramos essa transição, com as configurações axiais traçadas em dois diferentes estilos. Veja também a Figura 3.20 para uma ilustração da transição na superfície de Lie-Cartan.

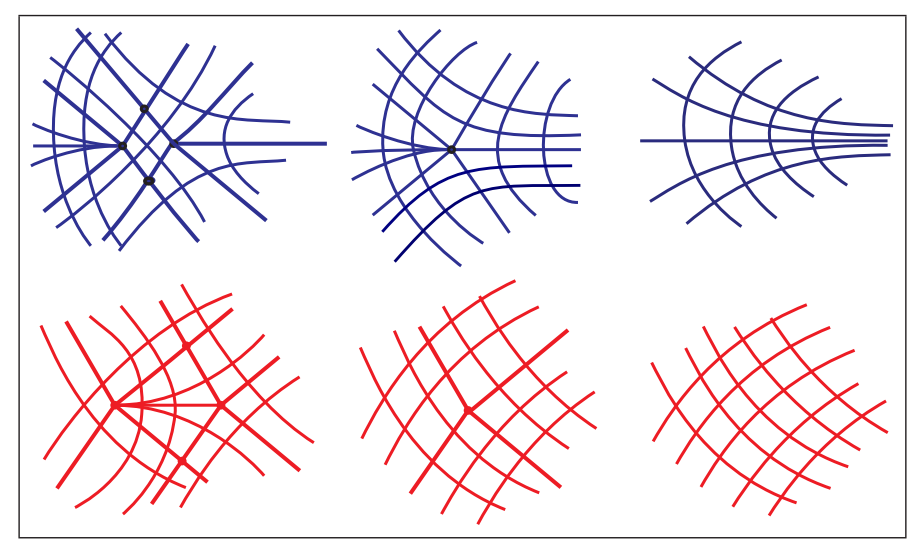

Figura 3.18: Ponto Axiumbílico $E_{45}^{1}$. Os pontos $E_{4}$ and $E_{5}$ colidem em um ponto tipo $E_{45}^{1}$, e após são eliminados e não há pontos axiumbílicos na vizinhança. 


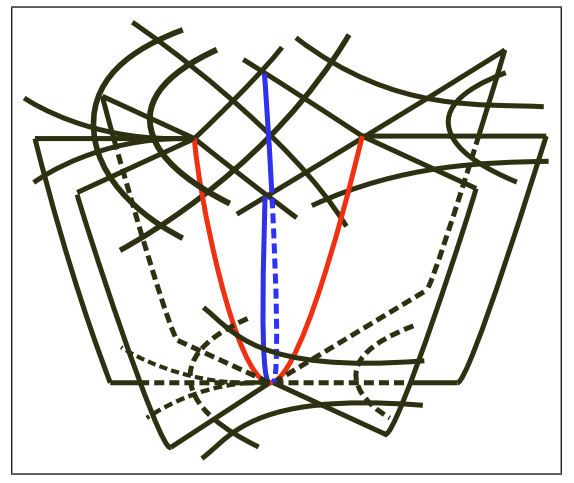

Figura 3.19: Diagrama de Bifurcação da configuração axial próximo do ponto axiumbílico $E_{45}^{1}$ e a estrutura de separatrizes.

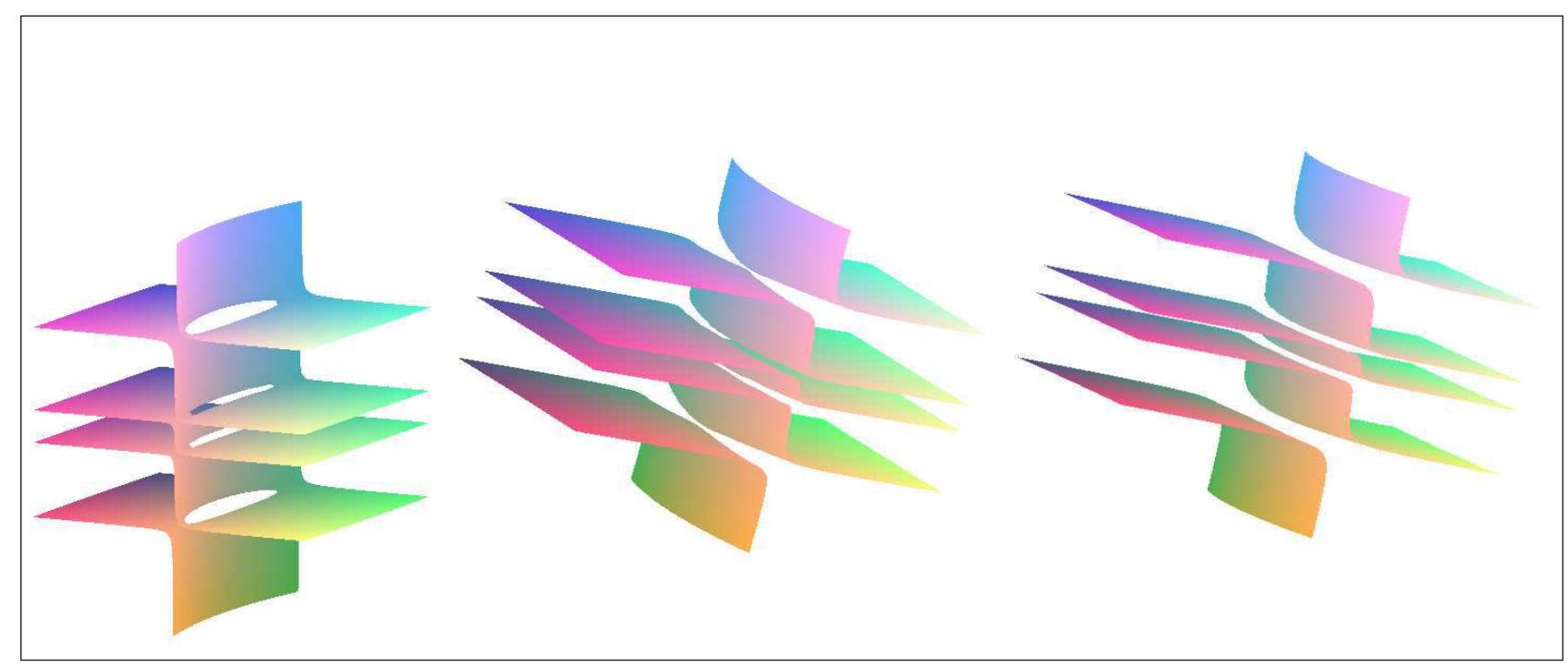

Figura 3.20: Superfície de Lie-Cartan. A esquerda, com dois pontos axiumbílicos, ao centro com quatro pontos singulares, e à direita os quatro níveis regulares.

Teorema 3.11. No espaço das aplicações suaves $M \times \mathbb{R} \longrightarrow \mathbb{R}^{4}$ que são imersões com relação à primeira variável, o conjunto das quais tem todos seus pontos axiumbílicos genéricos (de tipo $E_{3}, E_{4}$ and $E_{5}$ ) ou, transversalmente, de tipos $E_{34}^{1}$ and $E_{45}^{1}$ é aberto e denso. Além disso, para tais familias, os pontos axiumbílicos descrevem uma curva regular em $M \times \mathbb{R}$ cuja projeção em $\mathbb{R}$ possui apenas pontos críticos não-degenerados em $E_{45}^{1}$ e os pontos regulares da projeção é uma coleção de arcos por pontos $E_{34}^{1}$, com um fecho comum de arcos de $E_{3}$ e $E_{4}$.

Teorema 3.11 segue da análise feita nos Teoremas 3.4, 3.10 e da aplicação do Teorema de Transversalidade de Thom (Teorema A.8, Apêndice A) à subvariedade de 4 jatos de imersões em pontos axiumbílicos, estratificada pelos pontos axiumbílicos genéricos, pelos pontos $E_{34}^{1}$ e $E_{45}^{1}$, e por seus complementos. Efetuamos a estratificação na Seção 3.3. 


\subsection{Transversalidade e Estratificação}

Considere o espaço $J^{k}\left(M, \mathbb{R}^{4}\right)$ de $k$-jatos de imersões $\alpha$ de uma superfície compacta orientada $M$ em $\mathbb{R}^{4}$, dotado da estrutura de fibrado principal: a base é $M$; a fibra é o espaço $\mathbb{R}^{4} \times J^{k}(2,4)$, onde $J^{k}(2,4)$ é o espaço de $k$-jatos de imersões de $\mathbb{R}^{2}$ em $\mathbb{R}^{4}$, preservando as respectivas origens. O grupo estrutural, $\mathbb{A}_{+}^{k}$, é o produto do grupo de $\mathcal{L}_{+}^{k}(2,2)$ de $k$-jatos de difeomorfosmos de $\mathbb{R}^{2}$ que preservam a origem e a orientação, agindo à direita por mudança de coordenadas, e o grupo $\mathbb{R}^{4} \times \mathcal{O}_{+}(4,4)$ de isometrias positivas, agindo à esquerda, consistindo de uma translação, tomada como um vetor no primeiro fator, e uma rotação positiva de $\mathbb{R}^{4}$, tomada no segundo fator. Denote por $\Pi_{k, l}, k \leq l$ a projeção de $J^{l}(2,4)$ em $J^{k}(2,4)$. Sabemos que a ação de grupo comuta com projeções.

Definição 3.12. Definimos acima a estratificação axiumbílica canônica de $J^{4}(2,4)$. O termo canônico significa que os estratos são invariantes pela ação do grupo $\mathbb{A}_{+}^{k}=\mathcal{O}_{+}(4,4) \times$ $\mathcal{L}_{+}^{k}(2,2)$.

1) Jatos Axiumbílicos: $\mathcal{U}^{4}$, aqueles na órbita de $j^{4}(x, y, R(x, y), S(x, y))$, com $R$ e $S$ como nas equações (1.11) e (1.12) satisfazendo a condição de axiumbílico definida em termos de $j^{2} R(0)$ and $j^{2} S(0)$. Esta é uma subvariedade fechada de codimensão 2 .

2) Jatos Não-Axiumbílicos: $(\mathcal{N U})^{4}$ é o complemento de $\mathcal{U}^{4}$. Esta é uma subvariedade aberta de codimensão 0 .

3) Jatos Axiumbílicos Não-Estáveis: $(\mathcal{N E})^{4}$, na órbita dos jatos axiumbílicos para os quais:

- $T=\left(\alpha_{1} \alpha_{4}-\alpha_{2} \alpha_{3}\right)\left(r^{2}+s^{2}\right)=0$ ou

- $T \neq 0$ e as condições que caracterizam os pontos axiumbílicos $E_{3}$ e $E_{4}$, no Teorema 2.4, falham.

Esta é uma subvariedade fechada de codimensão 3, a qual pode ser expressa pela união dos seguintes estratos invariantes:

3.1) Jatos $N \tilde{a} o$-Transversais: $\mathcal{E}_{45}^{1}$ para os quais $T=0$ e $\chi \neq 0$. Este tem codimensão 3.

3.2) Jatos Duplo-Transversais: $\left(\mathcal{E}_{34}^{1}\right)^{4}$, o campo de Lie-Cartan tem uma sela-nó quadrática na linha projetiva a qual é caracterizada pelo Teorema 3.4. Tem codimensão 3.

4) Jatos Axiumbílicos Estáveis: $\mathcal{U E}^{4}$, o complementar em $\mathcal{U}^{4}$ de $\mathcal{N E}^{4}$.

Proposição 3.13. No espaço de famílias a um-parâmetro de imersões, aquelas cuja extensão do 4-jato são transversais à estratificação axiumbílica canônica é aberto e denso.

Demonstração: Segue do Teorema de Transversalidade de Thom (Teorema A.8, Apêndice A). 


\section{Capítulo 4}

\section{Ciclos Axiais Principais e Genericidade}

Trataremos neste capítulo dos ciclos axiais principais, os quais são linhas periódicas de curvatura axial principal (Definição 4.1). Iniciamos o capítulo utilizando resultados de Garcia e Sotomayor [42] a fim de parametrizar a vizinhança de um ciclo axial principal, para daí definir a Transformação de Primeiro Retorno (Definição 4.4) e, por meio da primeira e segunda derivadas desta transformação, vamos definir quando estes ciclos são hiperbólicos e semi-hiperbólicos (Definição 4.5).

Em termos dos coeficientes da equação diferencial das linhas axiais (Proposição 1.4), vamos exprimir as fórmulas da primeira e segunda derivadas da transformação de Poincaré (Proposição 4.6, Proposição 4.7). Obtendo estas fórmulas, efetuaremos o cálculo em termos da parametrização descrita na Proposição 4.3, e assim obteremos as expressões da primeira e segunda derivadas da transformação de primeiro retorno em termos da parametrização da vizinhança do ciclo (Proposição 4.8, Proposição 4.10).

Com as fórmulas que deduzimos, e fazendo uso de uma propriedade geométrica a qual provamos ser genérica ao longo dos ciclos axiais principais:

- Mostraremos no Teorema 4.9 que toda imersão de uma superfície em $\mathbb{R}^{4}$ com ciclo axial principal pode ser aproximada por uma imersão que torna tal ciclo axial principal hiperbólico.

- Mostraremos no Teorema 4.11 que toda imersão de uma superfície em $\mathbb{R}^{4}$ com ciclo axial principal não-hiperbólico pode ser aproximada por uma imersão que torna tal ciclo axial principal semi-hiperbólico.

Definição 4.1. Um ciclo axial principal é linha periódica de curvatura axial principal.

Lema 4.2 ( [42] pg. 128). Seja $\gamma: I \longrightarrow M$ ciclo axial parametrizado pelo comprimento de arco u. Então, ao longo de $\gamma$ está definido um referencial ortonormal positivo de Darboux $\left\{T_{1}, T_{2}, N_{1}, N_{2}\right\}$, sendo $T_{1}(u)=\gamma^{\prime}(u),\left\{N_{1}, N_{2}\right\}$, um referencial ortonormal do 


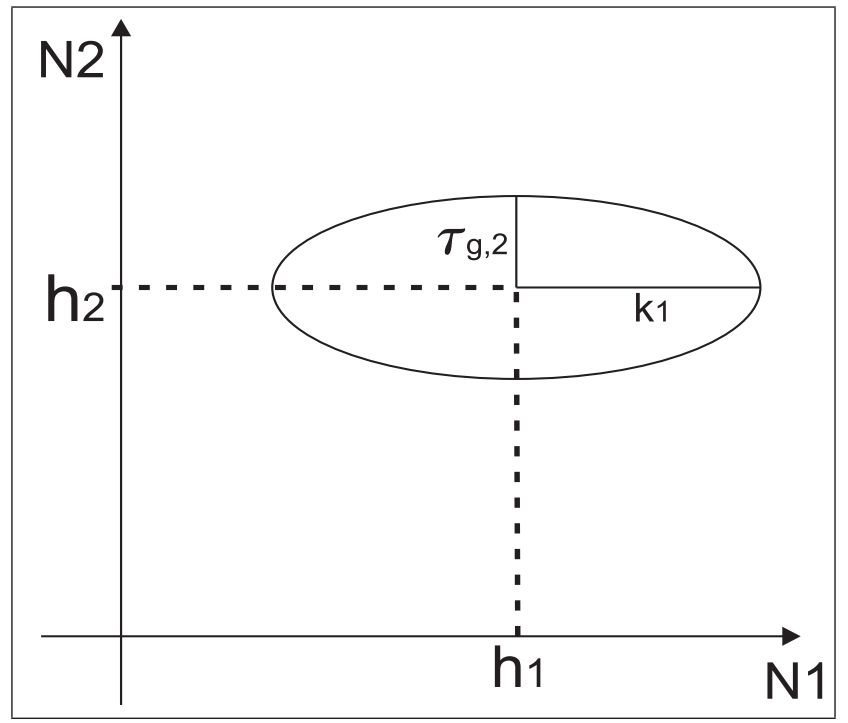

Figura 4.1: Ilustração da Elipse de Curvatura sobre o ciclo axial segundo a parametrização da Proposição 4.3

plano normal associado à elipse de curvatura com $N_{2}=T_{1} \wedge T_{2} \wedge N_{1}$ o qual satisfaz:

$$
\begin{aligned}
T_{1}^{\prime} & =k_{g} T_{2}+\left(h_{1}+k_{1}\right) N_{1}+h_{2} N_{2}, \\
T_{2}^{\prime} & =-k_{g} T_{1}+\tau_{g, 1} N_{1}+\tau_{g, 2} N_{2}, \\
N_{1}^{\prime} & =-\left(h_{1}+k_{1}\right) T_{1}-\tau_{g, 1} T_{2}+\tau_{n} N_{2}, \\
N_{2}^{\prime} & =-h_{2} T_{1}-\tau_{g, 2} T_{2}-\tau_{n} N_{1},
\end{aligned}
$$

onde $H=h_{1} N_{1}+h_{2} N_{2}$ é o vetor curvatura média, $\tau_{g}=\tau_{g, 1} N_{1}+\tau_{g, 2} N_{2}$ é o vetor torção geodésica, $k_{n}-H=k_{1} N_{1}, k_{1} \geq 0$ é o semi-eixo principal da elipse de curvatura $e$ $\tau_{n}=<N_{1}^{\prime}, N_{2}>$ é a torsão normal do referencial $\left\{N_{1}, N_{2}\right\}$.

Proposição 4.3 ( [42] pg. 129). Seja $\gamma$ um ciclo axial da configuração axial principal $\mathcal{P}_{\alpha}$ parametrizado pelo comprimento de arco u e comprimento $L$, e $\left\{T_{1}, T_{2}, N_{1}, N_{2}\right\}$ referencial positivo ortogonal de Darboux ao longo de $\gamma$. Então a expressão:

$$
\begin{aligned}
\alpha(u, v) & =\gamma(u)+v T_{2}(u) \\
& +\left[\frac{\left(h_{1}(u)-k_{1}(u)\right)}{2} v^{2}+A_{3}(u) \frac{v^{3}}{6}+A_{4}(u) \frac{v^{4}}{24}+A_{5}(u, v) \frac{v^{5}}{120}\right] N_{1}(u) \\
& +\left[\frac{h_{2}(u)}{2} v^{2}+B_{3}(u) \frac{v^{3}}{6}+B_{4}(u) \frac{v^{4}}{24}+B_{5}(u, v) \frac{v^{5}}{120}\right] N_{2}(u)
\end{aligned}
$$

define uma parametrização local, L-periódica em u, de classe $\mathcal{C}^{r-5}$ em uma vizinhança de $\gamma$.

Além disso, $\left(k_{1}^{2}-\tau_{g, 2}^{2}\right)$ é a diferença entre os quadrados dos eixos da elipse de curvatura $\varepsilon_{\alpha}$. 


\subsection{Primeira e Segunda Derivadas da Aplicação de Poincaré}

Considere a parametrização local da superfície dada pela Proposição 4.3. Em $p=$ $\alpha(0,0)=\gamma(0)$, considere uma seção transversal local $\Gamma$ a $\gamma$. Pelo teorema do fluxo tubular, para cada ponto $q \in \Gamma$ suficiente próximo de $p$, a única curva solução da equação (1.4) passando por $q$ permanece na vizinhança de $\gamma$.

Definição 4.4. Definimos a Aplicação de Primeiro Retorno $\Pi(q)$ como o primeiro ponto em que a solução da equação (1.4), partindo de $q$, volta a interceptar a seção $\Gamma$. Esta $\Pi:(\Gamma, p) \longrightarrow(\Gamma, p)$ também é denominada Transformação de Poincaré.

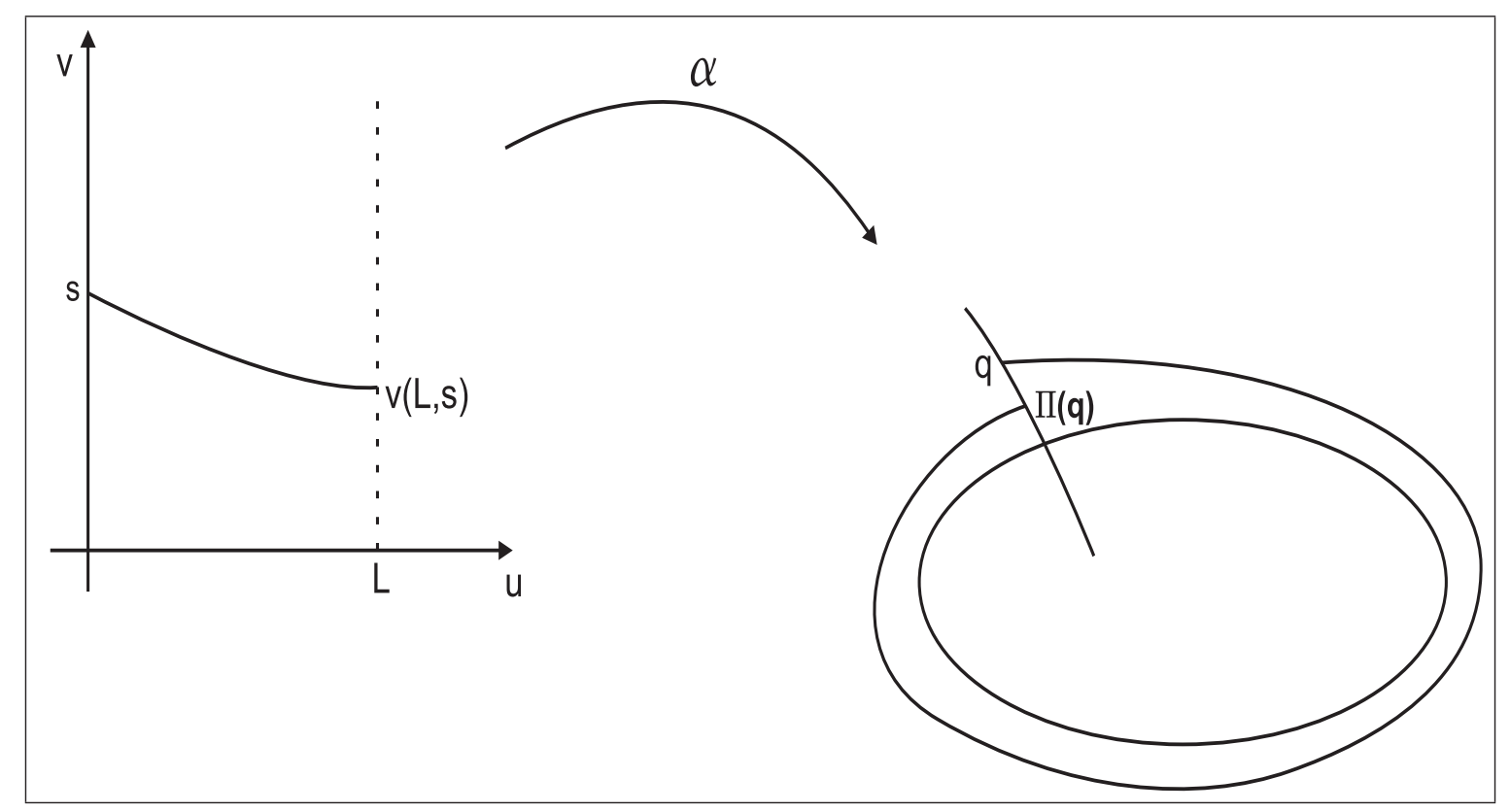

Figura 4.2: Parametrização local em torno do ciclo axial principal, ilustrando a aplicação de primeiro retorno.

Sendo $\gamma$ a curva coordenada $v=0$ e $\Gamma$ a curva coordenada $u=0$, considere o ponto $q=\alpha(0, s)$. A Transformação de Poincaré é dada então por

$$
\Pi(q)=\alpha(L, v(L, s))
$$

Assim, considerando

$$
\pi(s)=v(L, s)
$$

de modo que

$$
\Pi(q)=\alpha(L, \pi(s)),
$$

temos

$$
\pi^{\prime}(s)=\frac{\partial}{\partial s} v(L, s) .
$$


Assim

$$
\pi^{\prime}(0)=\left.\frac{\partial}{\partial s} v(L, s)\right|_{s=0}
$$

e

$$
\pi^{\prime \prime}(0)=\left.\frac{\partial^{2}}{\partial s^{2}} v(L, s)\right|_{s=0}
$$

Definição 4.5. O ciclo axial $\gamma$ é dito hiperbólico se $\pi^{\prime}(0) \neq 1$, e é semi-hiperbólico se $\pi^{\prime}(0)=1$ e $\pi^{\prime \prime}(0) \neq 0$.

Proposição 4.6. Seja $\gamma$ ciclo axial principal parametrizado pelo comprimento de arco u e comprimento L. Considere uma parametrização local da vizinhança de $\gamma$ dada pela Proposição 4.3 .Se a equação diferencial das linhas axiais é dada por

$$
a_{4}(u, v)\left(\frac{\partial v}{\partial u}\right)^{4}+a_{3}(u, v)\left(\frac{\partial v}{\partial u}\right)^{3}+a_{2}(u, v)\left(\frac{\partial v}{\partial u}\right)^{2}+a_{1}(u, v) \frac{\partial v}{\partial u}+a_{0}(u, v)=0
$$

então a primeira derivada da transformação de Poincaré tem a expressão:

$$
\pi^{\prime}(0)=\exp \left(\int_{0}^{L}-\frac{a_{0_{v}}(u, 0)}{a_{1}(u, 0)} d u\right) \text {. }
$$

Demonstração: Conforme ilustração na Figura 4.2, seja $\alpha(0,0)=\gamma(0)=p, \gamma$ a curva coordenada $\{v=0\}, \Gamma$ a curva coordenada $\{u=0\}$ e $q=\alpha(0, s) \in \Gamma$. Assim, uma solução da equação (4.1) passando por $q$ satisfaz

$$
\begin{array}{rlc}
a_{4}(u, v(u, s))\left(\frac{\partial v}{\partial u}\right)^{4} & + & a_{3}(u, v(u, s))\left(\frac{\partial v}{\partial u}\right)^{3} \\
& + & a_{2}(u, v(u, s))\left(\frac{\partial v}{\partial u}\right)^{2} \\
& + & a_{1}(u, v(u, s)) \frac{\partial v}{\partial u} \\
+ & a_{0}(u, v(u, s)) \\
= & 0,
\end{array}
$$

$\operatorname{com} v(0, s)=s$.

Abaixo derivamos a Equação (4.3) com relação a $s$. Para simplificar a notação, $a_{i}$ denotará 
$a_{i}(u, v)$ e $v$ denotará $v(u, s)$ :

$$
\begin{aligned}
{\left[\frac{\partial a_{4}}{\partial v} \cdot \frac{\partial v}{\partial s}\right] \cdot\left(\frac{\partial v}{\partial u}\right)^{4}+} & {\left[\frac{\partial a_{3}}{\partial v} \cdot \frac{\partial v}{\partial s}+4 \cdot a_{4} \cdot \frac{\partial^{2} v}{\partial u \partial s}\right] \cdot\left(\frac{\partial v}{\partial u}\right)^{3} } \\
+ & {\left[\frac{\partial a_{2}}{\partial v} \cdot \frac{\partial v}{\partial s}+3 \cdot a_{3} \cdot \frac{\partial^{2} v}{\partial u \partial s}\right] \cdot\left(\frac{\partial v}{\partial u}\right)^{2} } \\
+ & {\left[\frac{\partial a_{1}}{\partial v} \cdot \frac{\partial v}{\partial s}+2 \cdot a_{2} \cdot \frac{\partial^{2} v}{\partial u \partial s}\right] \cdot\left(\frac{\partial v}{\partial u}\right) } \\
+ & {\left[\frac{\partial a_{0}}{\partial v} \cdot \frac{\partial v}{\partial s}+a_{1} \cdot \frac{\partial^{2} v}{\partial u \partial s}\right] } \\
& =0
\end{aligned}
$$

Como $\left.\frac{\partial v}{\partial u}\right|_{s=0}=0$, segue que

$$
a_{1} \cdot \frac{\partial^{2}}{\partial u \partial s} v(u, s)+\frac{\partial a_{0}}{\partial v} \cdot \frac{\partial}{\partial s} v(u, s)=0
$$

a qual é uma equação diferencial de primeira ordem em $u$, cuja solução é

$$
\left.\frac{\partial}{\partial s} v(u, s)\right|_{s=0}=\exp \left(\int_{0}^{L}-\frac{\frac{\partial a_{0}}{\partial v}(u, 0)}{a_{1}(u, 0)} d u\right)
$$

Disso concluimos a equação (4.2).

Proposição 4.7. Sob as hipóteses da Proposição 4.6, a segunda derivada da transformação de Poincaré é expressa por:

$$
\pi^{\prime \prime}(0)=-\pi^{\prime}(0) \cdot \int_{0}^{L} \frac{1}{a_{1}^{3}}\left[2 a_{2}\left(a_{0_{v}}\right)^{2}-2 a_{1_{v}} a_{1} a_{0_{v}}+a_{1}^{2} a_{0_{v v}}\right] \cdot \eta(u) d u,
$$

onde

$$
\eta(u)=\exp \left(\int_{0}^{u}-\frac{\frac{\partial a_{0}}{\partial v}(t, 0)}{a_{1}(t, 0)} d t\right)
$$

Demonstração: Dando sequência à demonstração da Proposição 4.6, a segunda derivada da expressão $(*)$ com relação a $s$ é

$$
\Psi_{4} \cdot\left(\frac{\partial v}{\partial u}\right)^{4}+\Psi_{3} \cdot\left(\frac{\partial v}{\partial u}\right)^{3}+\Psi_{2} \cdot\left(\frac{\partial v}{\partial u}\right)^{2}+\Psi_{1} \cdot\left(\frac{\partial v}{\partial u}\right)+\Psi_{0}=0
$$

sendo

$$
\begin{aligned}
& \Psi_{4}=a_{4_{v v}} \cdot\left(v_{s}\right)^{2}+a_{4_{v}} \cdot v_{s s} \\
& \Psi_{3}=a_{3_{v v}} \cdot\left(v_{s}\right)^{2}+8 \cdot a_{4_{v}} \cdot v_{s} \cdot v_{u s}+4 \cdot a_{4} \cdot v_{u s s}+a_{3_{v}} \cdot v_{s s} \\
& \Psi_{2}=a_{2_{v v}} \cdot\left(v_{s}\right)^{2}+6 \cdot a_{3_{v}} \cdot v_{s} \cdot v_{u s}+12 \cdot a_{4} \cdot\left(v_{u s}\right)^{2}+3 \cdot a_{3} \cdot v_{u s s}+a_{2_{v}} \cdot v_{s s}
\end{aligned}
$$




$$
\begin{aligned}
& \Psi_{1}=a_{1_{v v}} \cdot\left(v_{s}\right)^{2}+4 \cdot a_{2_{v}} \cdot v_{s} \cdot v_{u s}+6 \cdot a_{3} \cdot\left(v_{u s}\right)^{2}+2 \cdot a_{2} \cdot v_{u s s}+a_{1_{v}} \cdot v_{s s} \\
& \Psi_{0}=a_{0_{v v}} \cdot\left(v_{s}\right)^{2}+2 \cdot a_{1_{v}} \cdot v_{s} \cdot v_{u s}+2 \cdot a_{2} \cdot\left(v_{u s}\right)^{2}+a_{1} \cdot v_{u s s}+a_{0_{v}} \cdot v_{s s}
\end{aligned}
$$

Usando que $v_{u}=0$, segue:

$$
a_{0_{v v}} \cdot\left(v_{s}\right)^{2}+2 \cdot a_{1_{v}} \cdot v_{s} \cdot v_{u s}+2 \cdot a_{2} \cdot\left(v_{u s}\right)^{2}+a_{1} \cdot v_{u s s}+a_{0_{v}} \cdot v_{s s}=0
$$

Tomando $s=0$, e denotando $\eta(u)=v_{s}(u, 0)$ e $\nu(u)=v_{s s}(u, 0)$, temos pela equação (4.2) que $\pi^{\prime}(0)=\eta(L)$ e, segundo a notação utilizada, $\pi^{\prime \prime}(0)=\nu(L)$. Portanto, em $s=0$, a equação (4.6) fica

$$
a_{1} \nu^{\prime}+a_{0_{v}} \nu+2 a_{2}\left(\eta^{\prime}\right)^{2}+2 a_{1_{v}} \eta \eta^{\prime}+a_{0_{v v}} \eta^{2}=0
$$

a qual tem por solução:

$$
\pi^{\prime \prime}(0)=-\pi^{\prime}(0) \cdot \int_{0}^{L} \frac{1}{a_{1}^{3}}\left[2 a_{2} a_{0_{v}}^{2}-2 a_{1_{v}} a_{1} a_{0_{v}}+a_{1}^{2} a_{0_{v v}}\right] \cdot \eta(u) d u .
$$

\subsection{Genericidade de Ciclos Hiperbólicos}

Considere a parametrização que determinamos na Proposição 4.3

$$
\begin{aligned}
\alpha(u, v) & =\gamma(u)+v T_{2}(u) \\
& +\left[\frac{\left(h_{1}(u)-k_{1}(u)\right)}{2} v^{2}+A_{3}(u) \frac{v^{3}}{6}+A_{4}(u) \frac{v^{4}}{24}+A_{5}(u, v) \frac{v^{5}}{120}\right] N_{1}(u) \\
& +\left[\frac{h_{2}(u)}{2} v^{2}+B_{3}(u) \frac{v^{3}}{6}+B_{4}(u) \frac{v^{4}}{24}+B_{5}(u, v) \frac{v^{5}}{120}\right] N_{2}(u) .
\end{aligned}
$$

Vamos calcular a primeira e segunda formas fundamentais da superfície $\left(O_{2}(v)\right.$ denota os termos de ordem maior ou igual a $2 \mathrm{em} v$ ). No cálculo das derivadas, fazemos uso do Lema 4.2:

$$
\begin{aligned}
\alpha_{u}(u, v) & =\left[1-k_{g}(u) v+O_{2}(v)\right] \cdot T_{1}(u) \\
& +\left[O_{2}(v)\right] \cdot T_{2}(u) \\
& +\left[\tau_{g, 1}(u) v+O_{2}(v)\right] \cdot N_{1}(u) \\
& +\left[\tau_{g, 2}(u) v+O_{2}(v)\right] \cdot N_{2}(u),
\end{aligned}
$$




$$
\begin{aligned}
\alpha_{v}(u, v) & =T_{2}(u) \\
& +\left[\left(h_{1}(u)-k_{1}(u)\right) v+O_{2}(v)\right] \cdot N_{1}(u) \\
& +\left[h_{2}(u) v+O_{2}(v)\right] \cdot N_{2}(u) .
\end{aligned}
$$

Assim,

$$
\begin{aligned}
E(u, v)= & <\alpha_{u}, \alpha_{u}> \\
= & 1-2 k_{g}(u) v+O_{2}(v), \\
F(u, v) & =<\alpha_{u}, \alpha_{v}> \\
& =O_{2}(v), \\
G(u, v) & =<\alpha_{v}, \alpha_{v}> \\
& =1+O_{2}(v) .
\end{aligned}
$$

Prosseguindo os cálculos:

$$
\begin{aligned}
\alpha_{u u}(u, v)= & {\left[-\left(k_{g}^{\prime}(u)+\left(h_{1}(u)+k_{1}(u)\right) \tau_{g, 1}(u)+h_{2}(u) \tau_{g, 2}(u)\right) v+O_{2}(v)\right] \cdot T_{1}(u) } \\
+ & {\left[k_{g}(u)-\left(k_{g}^{2}(u)+\tau_{g, 1}^{2}(u)+\tau_{g, 2}^{2}(u)\right) v+O_{2}(v)\right] \cdot T_{2}(u) } \\
+ & \left\{h_{1}(u)+k_{1}(u)\right. \\
+ & {\left.\left[\tau_{g, 1}^{\prime}(u)-\left(h_{1}(u)+k_{1}(u)\right) k_{g}(u)-\tau_{n}(u) \tau_{g, 2}(u)\right] v+O_{2}(v)\right\} \cdot N_{1}(u) } \\
+ & {\left[h_{2}(u)+\left(\tau_{g, 2}^{\prime}(u)-h_{2}(u) k_{g}(u)+\tau_{n}(u) \tau_{g, 1}(u)\right) v+O_{2}(v)\right] \cdot N_{2}(u), } \\
& \\
\alpha_{u v}(u, v)= & {\left[-k_{g}(u)-\left(h_{1}^{2}(u)+h_{2}^{2}(u)-k_{1}^{2}(u)\right) v+O_{2}(v)\right] \cdot T_{1}(u) } \\
+ & {\left[-\left(\left(h_{1}(u)-k_{1}(u)\right) \tau_{g, 1}(u)+h_{2}(u) \tau_{g, 2}(u)\right) v+O_{2}(v)\right] \cdot T_{2}(u) } \\
& +\left[\tau_{g, 1}(u)+\left(h_{1}^{\prime}(u)-k_{1}^{\prime}(u)-h_{2}(u) \tau_{n}(u)\right) v+O_{2}(v)\right] \cdot N_{1}(u) \\
& +\left[\tau_{g, 2}(u)+\left(\left(h_{1}(u)-k_{1}(u)\right) \tau_{n}(u)+h_{2}^{\prime}(u)\right) v+O_{2}(v)\right] \cdot N_{2}(u), \\
& \quad\left[h_{2}(u)+B(u, v) v+O_{2}(v)\right] \cdot N_{2}(u) .
\end{aligned}
$$

Com isto, os coeficientes da segunda forma fundamental são: 


$$
\begin{aligned}
& e_{1}(u, v)=<\alpha_{u u}, N_{1}> \\
& =h_{1}(u)+k_{1}(u)+\left[\tau_{g, 1}^{\prime}(u)-\left(h_{1}(u)+k_{1}(u)\right) k_{g}(u)-\tau_{n}(u) \tau_{g, 2}(u)\right] v+O_{2}(v) \text {, } \\
& f_{1}(u, v)=\left\langle\alpha_{u v}, N_{1}>\right. \\
& =\tau_{g, 1}(u)+\left(h_{1}^{\prime}(u)-k_{1}^{\prime}(u)-h_{2}(u) \tau_{n}(u)\right) v+O_{2}(v), \\
& g_{1}(u, v)=<\alpha_{v v}, N_{1}> \\
& =h_{1}(u)-k_{1}(u)+A(u, v) v+O_{2}(v) \text {, } \\
& e_{2}(u, v)=\left\langle\alpha_{u u}, N_{2}>\right. \\
& =h_{2}(u)+\left(\tau_{g, 2}^{\prime}(u)-h_{2}(u) k_{g}(u)+\tau_{n}(u) \tau_{g, 1}(u)\right) v+O_{2}(v), \\
& f_{2}(u, v)=<\alpha_{u v}, N_{2}> \\
& =\tau_{g, 2}(u)+\left(\left(h_{1}(u)-k_{1}(u)\right) \tau_{n}(u)+h_{2}^{\prime}(u)\right) v+O_{2}(v) \text {, } \\
& g_{2}(u, v)=\left\langle\alpha_{v v}, N_{2}>\right. \\
& =h_{2}(u)+B(u, v) v+O_{2}(v) \text {. }
\end{aligned}
$$

Proposição 4.8. Seja $\gamma$ um ciclo axial principal da configuração principal $\mathcal{P}_{\alpha}$, parametrizado pelo comprimento de arco u e comprimento L conforme a Proposição 4.3. Então a primeira derivada da aplicação de retorno $\pi$ é dada por:

$$
\ln \pi^{\prime}(0)=-\frac{1}{4} \int_{0}^{L} \frac{1}{k_{1}^{2}-\tau_{g, 2}^{2}} \cdot\left[\left(B_{3}+\tau_{g, 2}^{\prime}\right) \cdot \tau_{g, 2}+2\left(h_{2} \tau_{n}-h_{1}^{\prime}\right) k_{1}\right] d u \text {. }
$$

Demonstração: Nesta demonstração, vamos tomar a parametrização dada na Proposição 4.3, calcular os coeficientes da primeira e segunda formas fundamentais e, obtendo as expressões de $a_{0}$ e $a_{1}$ dadas na Proposição 1.4, substituir na equação (4.2), a fim de obter uma expressão de $\pi^{\prime}(0)$ em função da parametrização efetuada. Ao longo da linha $\{v=0\}$, temos que:

$$
\begin{gathered}
E(u, 0)=1, \quad F(u, 0)=0, \quad G(u, 0)=1, \\
e_{1}(u, 0)=h_{1}+k_{1}, \quad f_{1}(u, 0)=\tau_{g, 1}, \quad g_{1}(u, 0)=h_{1}-k_{1},
\end{gathered}
$$




$$
e_{2}(u, 0)=h_{2}, \quad f_{2}(u, 0)=\tau_{g, 2}, \quad g_{2}(u, 0)=h_{2} .
$$

Pela Proposição 1.4, temos

$$
\begin{aligned}
a_{0} & =4 F\left(E G-2 F^{2}\right)\left(e_{1}^{2}+e_{2}^{2}\right) \\
& -4 E\left(E G-4 F^{2}\right)\left(e_{1} f_{1}+e_{2} f_{2}\right) \\
& +4 E^{3}\left(f_{1} g_{1}+f_{2} g_{2}\right) \\
& -4 E^{2} F\left(e_{1} g_{1}+e_{2} g_{2}\right) \\
& -8 E^{2} F\left(f_{1}^{2}+f_{2}^{2}\right),
\end{aligned}
$$

e

$$
\begin{aligned}
a_{1} & =4 G\left(E G-4 F^{2}\right)\left(e_{1}^{2}+e_{2}^{2}\right) \\
& +32 E F G\left(e_{1} f_{1}+e_{2} f_{2}\right) \\
& +4 E^{3}\left(g_{1}^{2}+g_{2}^{2}\right) \\
& -8 E^{2} G\left(e_{1} g_{1}+e_{2} g_{2}\right) \\
& -16 E^{2} G\left(f_{1}^{2}+f_{2}^{2}\right) .
\end{aligned}
$$

Se admitirmos que a linha $\{v=0\}$ seja uma linha axial principal, temos que $a_{0}(u, 0)=0$ e $a_{1}(u, 0) \neq 0$. Efetuando as substituições convenientes, obtemos

$$
a_{0}(u, 0)=-8 \tau_{g, 1} k_{1}
$$

de onde concluimos que $\tau_{g, 1}=0$, visto que $k_{1}$ é o semi-eixo principal da elipse de curvatura o qual não se anula (Figura 4.1). Resulta então que

$$
a_{1}(u, 0)=16 \cdot\left[k_{1}^{2}-\tau_{g, 2}^{2}\right]
$$

Portanto,

$$
\left(k_{1}^{2}-\tau_{g, 2}^{2}\right) \neq 0
$$

Calculando a derivada parcial de $a_{0}(u, v)$ com relação a $v$, e tomando $v=0$, temos:

$$
\begin{aligned}
a_{0_{v}}(u, 0) & =8 \cdot k_{1}\left(h_{2} \tau_{n}+k_{1}^{\prime}-h_{1}^{\prime}\right)+4 \cdot \tau_{g, 2}\left(B_{3}-\tau_{g, 2}^{\prime}\right) \\
& +\left[32 k_{g}(u) k_{1}(u)+4 A_{3}(u)-4 \tau_{g, 1}^{\prime}(u)\right] \cdot \tau_{g, 1}(u) .
\end{aligned}
$$

Como $\tau_{g, 1}(u)=0$, segue que

$$
a_{0_{v}}(u, 0)=8 \cdot k_{1}\left(h_{2} \tau_{n}+k_{1}^{\prime}-h_{1}^{\prime}\right)+4 \cdot \tau_{g, 2}\left(B_{3}-\tau_{g, 2}^{\prime}\right) .
$$


Assim,

$$
-\frac{a_{0_{v}}}{a_{1}}=-\frac{1}{4} \cdot \frac{1}{k_{1}^{2}-\tau_{g, 2}^{2}} \cdot\left[\left(B_{3}-\tau_{g, 2}^{\prime}\right) \cdot \tau_{g, 2}+\left(k_{1}^{\prime}-h_{1}^{\prime}+h_{2} \tau_{n}\right) \cdot 2 k_{1}\right]
$$

Efetuando

$$
\left(B_{3}-\tau_{g, 2}^{\prime}\right) \tau_{g, 2}+\left(k_{1}^{\prime}-h_{1}^{\prime}+h_{2} \tau_{n}\right) 2 k_{1}=\left(B_{3}+\tau_{g, 2}^{\prime}\right) \tau_{g, 2}+\left(h_{2} \tau_{n}-h_{1}^{\prime}\right) 2 k_{1}+2 k_{1} k_{1}^{\prime}-2 \tau_{g, 2} \tau_{g, 2}^{\prime}
$$

temos a forma conveniente

$$
-\frac{a_{0_{v}}}{a_{1}}=-\frac{1}{4} \cdot\left[\frac{1}{k_{1}^{2}-\tau_{g, 2}^{2}} \cdot\left[\left(B_{3}+\tau_{g, 2}^{\prime}\right) \cdot \tau_{g, 2}+2\left(h_{2} \tau_{n}-h_{1}^{\prime}\right) k_{1}\right]\right]-\frac{1}{4} \cdot \frac{2 k_{1} k_{1}^{\prime}-2 \tau_{g, 2} \tau_{g, 2}^{\prime}}{k_{1}^{2}-\tau_{g, 2}^{2}} .
$$

De acordo com a Equação (4.2), integrando ambos os lados de 0 a $L$ temos:

$$
\begin{aligned}
\int_{0}^{L}-\frac{a_{0_{v}}}{a_{1}} d u & =-\frac{1}{4} \int_{0}^{L} \frac{1}{k_{1}^{2}-\tau_{g, 2}^{2}} \cdot\left[\left(B_{3}+\tau_{g, 2}^{\prime}\right) \cdot \tau_{g, 2}+2\left(h_{2} \tau_{n}-h_{1}^{\prime}\right) k_{1}\right] d u \\
& -\frac{1}{4} \int_{0}^{L} \frac{2 k_{1} k_{1}^{\prime}-2 \tau_{g, 2} \tau_{g, 2}^{\prime}}{k_{1}^{2}-\tau_{g, 2}^{2}} d u .
\end{aligned}
$$

Como

$$
\int_{0}^{L} \frac{2 k_{1} k_{1}^{\prime}-2 \tau_{g, 2} \tau_{g, 2}^{\prime}}{k_{1}^{2}-\tau_{g, 2}^{2}} d u=\int_{0}^{L} d\left(\ln \left[k_{1}^{2}-\tau_{g, 2}^{2}\right]\right)=0,
$$

resulta a fórmula

$$
\ln \pi^{\prime}(0)=-\frac{1}{4} \int_{0}^{L} \frac{1}{k_{1}^{2}-\tau_{g, 2}^{2}} \cdot\left[\left(B_{3}+\tau_{g, 2}^{\prime}\right) \cdot \tau_{g, 2}+2\left(h_{2} \tau_{n}-h_{1}^{\prime}\right) k_{1}\right] d u
$$

Teorema 4.9. Seja $\alpha: M^{2} \longrightarrow \mathbb{R}^{4}$ imersão de classe $\mathcal{C}^{r}, r \geq 6$ e $\gamma$ ciclo axial principal de $\alpha$, parametrizado pelo comprimento de arco u e comprimento L. Considere a carta $(u, v)$ conforme a Proposição 4.3 e a perturbação

$$
\alpha_{\varepsilon}(u, v)=\alpha(u, v)+\varepsilon\left[\frac{\tau_{g, 2}}{6} v^{3}\right] \delta(v) N_{2}(u)
$$

onde $\delta=1$ na vizinhança de $v=0$ com suporte compacto e assumimos $\tau_{g, 2} \neq 0$. Então, $\gamma$ é um ciclo axial principal de $\alpha_{\varepsilon}$, para $\varepsilon$ suficientemente pequeno, o qual é hiperbólico para $\alpha_{\varepsilon}, \quad \varepsilon \neq 0$. 
Demonstração: Temos que

$$
\begin{aligned}
\alpha_{\varepsilon}(u, v) & =\gamma(u)+v T_{2}(u) \\
& +\left[\frac{\left(h_{1}(u)-k_{1}(u)\right)}{2} v^{2}+A_{3}(u) \frac{v^{3}}{6}+A_{4}(u) \frac{v^{4}}{24}+A_{5}(u, v) \frac{v^{5}}{120}\right] N_{1}(u) \\
& +\left[\frac{h_{2}(u)}{2} v^{2}+\left[B_{3}(u)+\varepsilon \tau_{g, 2}(u)\right] \frac{v^{3}}{6}+B_{4}(u) \frac{v^{4}}{24}+B_{5}(u, v) \frac{v^{5}}{120}\right] N_{2}(u) .
\end{aligned}
$$

De acordo com a fórmula da derivada primeira, enunciada na Proposição 4.8,

$$
\ln \pi^{\prime}(0)=-\frac{1}{4} \int_{0}^{L} \frac{1}{k_{1}^{2}-\tau_{g, 2}^{2}} \cdot\left[\left(B_{3}+\varepsilon \tau_{g, 2}+\tau_{g, 2}^{\prime}\right) \cdot \tau_{g, 2}+2\left(h_{2} \tau_{n}-h_{1}^{\prime}\right) k_{1}\right] d u \text {. }
$$

Derivando com relação a $\varepsilon$ temos:

$$
\left.\frac{d}{d \varepsilon} \ln \pi^{\prime}(0)\right|_{\varepsilon=0}=-\frac{1}{4} \int_{0}^{L} \frac{\tau_{g, 2}^{2}}{k_{1}^{2}-\tau_{g, 2}^{2}} d u \neq 0
$$

Com o Teorema 4.9, garantimos que uma imersão que possui um ciclo axial principal $\gamma$ não-hiperbólico pode sempre ser aproximada por uma imersão na qual o ciclo axial principal $\gamma$ é hiperbólico.

\subsection{Genericidade de Ciclos Semi-Hiperbólicos}

Vamos estudar a segunda derivada da transformação de retorno para os ciclos principais não-hiperbólicos (os quais satisfazem $\pi^{\prime}(0)=1$ ). De fato, conforme a Equação (4.5), para um ciclo não-hiperbólico:

$$
\pi^{\prime \prime}(0)=-\int_{0}^{L} \frac{1}{a_{1}^{3}}\left[2 a_{2}\left(a_{0_{v}}\right)^{2}-2 a_{1_{v}} a_{1} a_{0_{v}}+a_{1}^{2} a_{0_{v v}}\right] \cdot \eta(u) d u,
$$

sendo

$$
\eta(u)=\exp \left(\int_{0}^{u}-\frac{\frac{\partial a_{0}}{\partial v}(t, 0)}{a_{1}(t, 0)} d t\right)
$$

Ao longo da curva $\{v=0\}$, temos que

$$
2 a_{2} a_{0_{v}}^{2}-2 a_{1_{v}} a_{1} a_{0_{v}}+a_{1}^{2} a_{0_{v v}}=1024\left(k_{1}^{2}-\tau_{g, 2}^{2}\right) \cdot \Phi,
$$

com

$$
\Phi=\Phi_{5} \tau_{g, 2}^{5}+\Phi_{4} \tau_{g, 2}^{4}+\Phi_{3} \tau_{g, 2}^{3}+\Phi_{2} \tau_{g, 2}^{2}+\Phi_{1} \tau_{g, 2}+\Phi_{0},
$$


onde:

$$
\begin{aligned}
\Phi_{5} & =3 h_{2}, \\
\Phi_{4} & =2 k_{g}^{\prime} \\
\Phi_{3} & =3 h_{2} h_{1}^{2}+\left[\tau_{n}^{\prime}-2 h_{2} k_{1}\right] h_{1}+3 h_{2}^{3}+\left[\tau_{n}^{2}-8 k_{1}^{2}\right] h_{2} \\
& +h_{2}^{\prime \prime}+\left[2 q-\tau_{n}^{\prime}\right] k_{1}-2 k_{g} \tau_{g, 2}^{\prime}+5 k_{g} B_{3}-B_{4}, \\
\Phi_{2} & =\left[-2 \tau_{n} \tau_{g, 2}+2 B_{3} \tau_{n}\right] h_{1}+\left[2 A_{3} \tau_{n}+10 k_{g} \tau_{n} k_{1}\right] h_{2} \\
& +\left[2 A_{3}^{\prime}-2 B_{3} \tau_{n}+10 k_{g}\left(k_{1}^{\prime}-h_{1}^{\prime}\right)\right] k_{1}+2 h_{2}^{\prime}\left[B_{3}-\tau_{g, 2}^{\prime}\right]+2 A_{3}\left[k_{1}^{\prime}-h_{1}^{\prime}\right]-2 k_{g}^{\prime} k_{1}^{2}, \\
\Phi_{1} & =5 h_{2} k_{1}^{4}+\left[\tau_{n}^{\prime}+2 h_{2} h_{1}-2 q\right] k_{1}^{3} \\
& +\left[4 \tau_{n}\left(h_{1}^{\prime}-k_{1}^{\prime}\right)-3 h_{2}\left(h_{1}^{2}+h_{2}^{2}\right)-2 \tau_{g, 2}^{\prime} k_{g}-5 h_{2} \tau_{n}^{2}-h_{1} \tau_{n}^{\prime}-h_{2}^{\prime \prime}+B_{4}\right] k_{1}^{2} \\
& +\left[8 h_{2}^{\prime}\left(k_{1}^{\prime}-h_{1}^{\prime}\right)+8 \tau_{n}\left(h_{2} h_{2}^{\prime}-h_{1} h_{1}^{\prime}\right)+8 \tau_{n}^{2} h_{1} h_{2}+2 A_{3}\left(B_{3}-\tau_{g, 2}^{\prime}\right)\right] k_{1}, \\
\Phi_{0} & =\left(A_{3}-k_{g} k_{1}\right)\left(h_{1}^{\prime}-k_{1}^{\prime}\right)+\left[k_{g} k_{1} h_{2}-k_{1} \tau_{g, 2}^{\prime}-h_{2} A_{3}+h_{1} \tau_{g, 2}-h_{1} B_{3}\right] \tau_{n} \\
& +h_{2}^{\prime}\left(\tau_{g, 2}^{\prime}-B_{3}\right)+A_{3}^{\prime} k_{1} .
\end{aligned}
$$

Como $a_{1}(u, 0)=16 \cdot\left(k_{1}^{2}-\tau_{g, 2}^{2}\right)$, segue que $a_{1}^{3}=4096\left(k_{1}^{2}-\tau_{g, 2}^{2}\right)^{3}$, e portanto

$$
\begin{aligned}
\frac{1}{a_{1}^{3}}\left[2 a_{2}\left(a_{0_{v}}\right)^{2}-2 a_{1_{v}} a_{1} a_{0_{v}}+a_{1}^{2} a_{0_{v v}}\right] & =\frac{1024\left(k_{1}^{2}-\tau_{g, 2}^{2}\right) \cdot \Phi}{4096\left(k_{1}^{2}-\tau_{g, 2}^{2}\right)^{3}} \\
& =\frac{1}{4} \cdot \frac{1}{\left(k_{1}^{2}-\tau_{g, 2}^{2}\right)^{2}} \cdot \Phi
\end{aligned}
$$

Disso enunciamos a Proposição 4.10.

Proposição 4.10. Seja $\gamma$ um ciclo axial principal da configuração principal $\mathcal{P}_{\alpha}$, parametrizado pelo comprimento de arco u e comprimento L, o qual é não-hiperbólico. Então a segunda derivada da aplicação de retorno $\pi$ é dada por:

$$
\pi^{\prime \prime}(0)=\frac{1}{4} \cdot \int_{0}^{L} \frac{\Phi}{\left(k_{1}^{2}-\tau_{g, 2}^{2}\right)^{2}} \cdot \eta(u) d u
$$

onde $\Phi$ é descrito na equação (4.9), e

$$
\eta(u)=\exp \left(-\frac{1}{4} \int_{0}^{u} \frac{1}{k_{1}^{2}-\tau_{g, 2}^{2}} \cdot\left[\left(B_{3}+\tau_{g, 2}^{\prime}\right) \cdot \tau_{g, 2}+2\left(h_{2} \tau_{n}-h_{1}^{\prime}\right) k_{1}\right] d t\right) .
$$

Teorema 4.11. Seja $\alpha: M^{2} \longrightarrow \mathbb{R}^{4}$ imersão de classe $\mathcal{C}^{r}, r \geq 6$ e $\gamma$ ciclo axial principal não-hiperbólico de $\alpha$, parametrizado pelo comprimento de arco u e comprimento $L$. Considere a carta $(u, v)$ conforme a Proposição 4.3 e a perturbação:

$$
\alpha_{\varepsilon}(u, v)=\alpha(u, v)+\varepsilon\left[\frac{\tau_{g, 2}}{24} v^{4}\right] \delta(v) N_{2}(u),
$$


onde $\delta=1$ na vizinhança de $v=0$ com suporte compacto e assumimos $\tau_{g, 2} \neq 0$. Então, $\gamma$ é um ciclo axial principal de $\alpha_{\varepsilon}$, para $\varepsilon$ suficientemente pequeno, o qual é semi-hiperbólico $\operatorname{para} \alpha_{\varepsilon}, \quad \varepsilon \neq 0$.

Demonstração: Temos que:

$$
\begin{aligned}
\alpha_{\varepsilon}(u, v) & =\gamma(u)+v T_{2}(u) \\
& +\left[\frac{\left(h_{1}(u)-k_{1}(u)\right)}{2} v^{2}+A_{3}(u) \frac{v^{3}}{6}+A_{4}(u) \frac{v^{4}}{24}+A_{5}(u, v) \frac{v^{5}}{120}\right] N_{1}(u) \\
& +\left[\frac{h_{2}(u)}{2} v^{2}+B_{3}(u) \frac{v^{3}}{6}+\left[B_{4}(u)+\varepsilon \tau_{g, 2}(u)\right] \frac{v^{4}}{24}+B_{5}(u, v) \frac{v^{5}}{120}\right] N_{2}(u) .
\end{aligned}
$$

Efetuando o cálculo da derivada primeira em $\alpha_{\varepsilon}$ de acordo com a Proposição 4.8, temos que

$$
\ln \pi_{\alpha_{\varepsilon}}^{\prime}(0)=-\frac{1}{4} \int_{0}^{L} \frac{1}{k_{1}^{2}-\tau_{g, 2}^{2}} \cdot\left[\left(B_{3}+\tau_{g, 2}^{\prime}\right) \cdot \tau_{g, 2}+2\left(h_{2} \tau_{n}-h_{1}^{\prime}\right) k_{1}\right] d u,
$$

e portanto $\pi_{\alpha_{\varepsilon}}^{\prime}(0)=\pi_{\alpha}^{\prime}(0)=1$.

Efetuando substituições convenientes na fórmula que fizemos descrevemos na Proposição 4.10, na carta $\alpha_{\varepsilon}$ a expressão da derivada segunda é dada por

$$
\pi^{\prime \prime}(0)=\frac{1}{4} \cdot \int_{0}^{L} \frac{1}{\left(k_{1}^{2}-\tau_{g, 2}^{2}\right)^{2}} \cdot\left[\Phi+\varepsilon \tau_{g, 2}^{2}\left(k_{1}^{2}-\tau_{g, 2}^{2}\right)\right] \cdot \eta(u) d u,
$$

sendo $\Phi$ descrito pela equação (4.9). Derivando com relação a $\varepsilon$, obtemos:

$$
\left.\frac{d}{d \varepsilon} \pi^{\prime \prime}(0)\right|_{\varepsilon=0}=\frac{1}{4} \int_{0}^{L} \frac{\tau_{g, 2}^{2}}{k_{1}^{2}-\tau_{g, 2}^{2}} \cdot \eta(u) d u \neq 0 .
$$

Com o Teorema 4.11, garantimos que uma imersão que possui um ciclo axial principal $\gamma$ não-hiperbólico e não semi-hiperbólico , pode sempre ser aproximada por uma imersão na qual o ciclo axial principal $\gamma$ é semi-hiperbólico.

\subsection{Sobre a Condição $\tau_{g, 2} \neq 0$}

Nos Teoremas 4.9 e 4.11, efetuamos a hipótese de que $\tau_{g, 2} \neq 0$. De acordo com a Proposição 4.3, $\tau_{g, 2}$ corresponde ao semi-eixo menor da elipse de curvatura ao longo do ciclo axial principal. A fim de garantir que $\tau_{g, 2}$ não se anule genericamente ao longo de um ciclo axial principal, vamos provar nesta seção que, ao longo de um ciclo axial principal, genericamente a elipse de curvatura tem área não nula.

De acordo com Ganchev e Milousheva [13], adaptado à notação que utilizamos ao longo deste texto (vide Seção 1.1), para uma imersão $\alpha: M^{2} \longrightarrow \mathbb{R}^{4}$ de uma superfície suave e 
orientada, seja $\left\{\alpha_{x}, \alpha_{y}\right\}$ referencial (não necessariamente ortonormal) de $T_{p} M$ e $\left\{N_{1}, N_{2}\right\}$ referencial ortonormal de $N_{p} M$, de modo que $\left\{\alpha_{x}, \alpha_{y}, N_{1}, N_{2}\right\}$ seja um referencial positivo.

Lembramos que $E=<\alpha_{u}, \alpha_{u}>, F=<\alpha_{u}, \alpha_{v}>$ e $G=<\alpha_{v}, \alpha_{v}>$ e denotamos por $W=\sqrt{E G-F^{2}}$. Além disso,

$$
\begin{aligned}
& \alpha_{u u}=\Gamma_{11}^{1} \alpha_{u}+\Gamma_{11}^{2} \alpha_{v}+e_{1} N_{1}+e_{2} N_{2}, \\
& \alpha_{u v}=\Gamma_{12}^{1} \alpha_{u}+\Gamma_{12}^{2} \alpha_{v}+f_{1} N_{1}+f_{2} N_{2}, \\
& \alpha_{v v}=\Gamma_{22}^{1} \alpha_{u}+\Gamma_{22}^{2} \alpha_{v}+g_{1} N_{1}+g_{2} N_{2},
\end{aligned}
$$

onde $\Gamma_{j k}^{i}, \quad i, j, k=1,2$ são os símbolos de Christoffel, a saber:

$$
\begin{array}{ll}
\Gamma_{11}^{1}=\left\langle\alpha_{u u}, \alpha_{u}\right\rangle, & \Gamma_{11}^{2}=\left\langle\alpha_{u u}, \alpha_{v}\right\rangle, \\
\Gamma_{12}^{1}=\left\langle\alpha_{u v}, \alpha_{u}\right\rangle, & \Gamma_{12}^{2}=\left\langle\alpha_{u v}, \alpha_{v}\right\rangle, \\
\Gamma_{22}^{1}=\left\langle\alpha_{v v}, \alpha_{u}\right\rangle, & \Gamma_{22}^{2}=\left\langle\alpha_{v v}, \alpha_{v}\right\rangle,
\end{array}
$$

e os coeficientes da segunda forma fundamental, para $i=1,2$, são

$$
e_{i}=\left\langle\alpha_{u u}, N_{i}\right\rangle, \quad f_{i}=\left\langle\alpha_{u v}, N_{i}\right\rangle, \quad g_{i}=\left\langle\alpha_{v v}, N_{i}\right\rangle
$$

Seja

$$
\Delta_{1}=\left|\begin{array}{cc}
e_{1} & e_{2} \\
f_{1} & f_{2}
\end{array}\right|, \quad \Delta_{2}=\left|\begin{array}{cc}
e_{1} & e_{2} \\
g_{1} & g_{2}
\end{array}\right|, \quad \Delta_{3}=\left|\begin{array}{ll}
f_{1} & f_{2} \\
g_{1} & g_{2}
\end{array}\right|,
$$

e

$$
L=\frac{2 \Delta_{1}}{W}, \quad M=\frac{\Delta_{2}}{W}, \quad N=\frac{2 \Delta_{3}}{W} .
$$

Definimos

$$
\tilde{\kappa}_{\alpha}=\frac{E N-2 F M+G L}{2\left(E G-F^{2}\right)},
$$

o qual é um invariante da superfície $M^{2}$ [13]. A área da elipse de curvatura de $\alpha$ em um ponto $\mathfrak{p}=(x, y) \in M^{2}$ é portanto dada por:

$$
A_{\alpha}(x, y)=\frac{\pi}{2} \cdot\left|\tilde{\kappa}_{\alpha}(x, y)\right|
$$

Seja

$$
\kappa_{\alpha}=E N-2 F M+G L .
$$

Lema 4.12. Para um conjunto residual em $\mathcal{I}^{r}\left(M^{2}, \mathbb{R}^{4}\right), r \geq 4$, a equação $\kappa_{\alpha}(x, y)=0$ define uma curva regular em $M^{2} \backslash \mathcal{U}_{\alpha}$.

Proposição 4.13. Para um conjunto residual em $\mathcal{I}^{r}\left(M^{2}, \mathbb{R}^{4}\right)$ e para toda $\gamma$ linha axial da imersão $\alpha: M^{2} \longrightarrow \mathbb{R}^{4}$, as curvas $\kappa_{\alpha}=0$ e $\gamma$ se encontram transversalmente, exceto em um número finito de pontos onde têm contato quadrático. 
Demonstração: Considere a aplicação $F: P M \times I^{r}\left(M^{2}, \mathbb{R}^{4}\right) \longrightarrow \mathbb{R}^{3}$ dada por

$$
F(x, y,[d x: d y], \alpha)=\left(\kappa_{\alpha}(x, y), \mathcal{G}_{\alpha}(x, y,[d x: d y]), H_{\alpha}(x, y)\right)
$$

sendo

$$
\mathcal{G}_{\alpha}(x, y,[d x: d y])=a_{4} d y^{4}+a_{3} d y^{3} d x+a_{2} d y^{2} d x^{2}+a_{1} d y d x^{3}+a_{0} d x^{4},
$$

a qual é descrita na Seção 1.2, e

$$
H_{\alpha}(x, y)=\mathcal{G}_{\alpha}\left(x, y,\left(\nabla \kappa_{\alpha}(x, y)\right)^{\perp}\right)
$$

De fato, se $F^{-1}(0,0,0)=\emptyset$, então as curvas se encontram transversalmente (Figura 4.3). No caso em que $F^{-1}(0,0,0) \neq \emptyset$ para alguma imersão $\alpha$, vamos considerar a expressão numa carta de Monge $(x, y, R, S)$ na vizinhança de um ponto $\mathfrak{p} \in F^{-1}(0,0,0)$

$$
\begin{aligned}
R(x, y) & =\frac{r_{20}}{2} x^{2}+r_{11} x y+\frac{r_{20}+r}{2} y^{2}+\frac{r_{30}}{6} x^{3}+\frac{r_{21}}{2} x^{2} y+\frac{r_{12}}{2} x y^{2}+\frac{r_{03}}{6} y^{3} \\
& +\frac{r_{40}}{24} x^{4}+\frac{r_{31}}{6} x^{3} y+\frac{r_{22}}{4} x^{2} y^{2}+\frac{r_{13}}{6} x y^{3}+\frac{r_{04}}{24} y^{4}+\text { h.o.t. }, \\
S(x, y) & =\frac{s_{20}}{2} x^{2}+s_{11} x y+\frac{s_{20}+s}{2} y^{2}+\frac{s_{30}}{6} x^{3}+\frac{s_{21}}{2} x^{2} y+\frac{s_{12}}{2} x y^{2}+\frac{s_{03}}{6} y^{3} \\
& +\frac{s_{40}}{24} x^{4}+\frac{s_{31}}{6} x^{3} y+\frac{s_{22}}{4} x^{2} y^{2}+\frac{s_{13}}{6} x y^{3}+\frac{s_{04}}{24} y^{4}+\text { h.o.t. }
\end{aligned}
$$

Temos:

$$
\kappa(0,0)=-r \cdot s_{11}+s \cdot r_{11}
$$

e

$$
\mathcal{G}(0,0,0)=r \cdot r_{11}+s \cdot s_{11}
$$

Portanto, $\kappa(0,0)=\mathcal{G}(0,0,0)=0$ se, e somente se,

$$
\left(r_{11}-s_{11}\right) \cdot r+\left(r_{11}+s_{11}\right) \cdot s=0
$$

o que ocorre quando:

$$
\left\{\begin{array}{l}
r_{11}=s_{11}=0 \\
\text { ou } \\
r=s=0 .
\end{array}\right.
$$

Para imersão $\alpha$, condição necessária e suficiente para que as curvas $\gamma$ e a curva $\kappa_{\alpha}=0$ tenham contato quadrático é que $\operatorname{det}\left(\operatorname{Jac}\left(F_{\alpha}\right)\right) \neq 0$.

De fato,

$$
\operatorname{Jac}\left(F_{\alpha}\right)=\left|\begin{array}{ccc}
\kappa_{x} & \kappa_{y} & 0 \\
\mathcal{G}_{x} & \mathcal{G}_{y} & \mathcal{G}_{p} \\
H_{x} & H_{y} & 0
\end{array}\right|
$$


e portanto

$$
\operatorname{Jac}\left(F_{\alpha}\right)=-\mathcal{G}_{p} \cdot\left(\kappa_{x} \cdot H_{y}-\kappa_{y} \cdot H_{x}\right) .
$$

Se $r_{11}=s_{11}=0$, então

$$
H_{\alpha}(0,0)=-\frac{\left(r^{2}+s^{2}\right)\left(r s_{21}-s r_{21}\right)}{\left(r s_{12}-s r_{12}\right)^{3}} \cdot\left[\left(s_{12}^{2}-s_{21}^{2}\right) r^{2}+2\left(r_{21} s_{21}-r_{12} s_{12}\right) r s+\left(r_{12}^{2}-r_{21}^{2}\right) s^{2}\right] .
$$

Sendo $H_{\alpha}(0,0)=0$, segue que

$$
\begin{aligned}
\operatorname{Jac}\left(F_{\alpha}\right) & =-\frac{s^{4}\left(r_{21}^{2}+s_{21}^{2}\right)}{s_{21}^{5}} \\
& \cdot\left[\left(3 r_{12}-2 r_{30}\right) s_{21}^{4}+\left(\left(-3 s_{12}+2 s_{30}\right) r_{21}-r_{31} s\right) s_{21}^{3}\right. \\
+ & \left(\left(3 r_{12}-2 r_{30}\right) r_{21}^{2}+s_{31} s r_{21}\right) s_{21}^{2} \\
+ & \left.\left(\left(-3 s_{12}+2 s_{30}\right) r_{21}^{3}-r_{31} s r_{21}^{2}\right) s_{21}+r_{21}^{3} s s_{31}\right] .
\end{aligned}
$$

Portanto, $\operatorname{det}\left(J a c\left(F_{\alpha}\right)\right)$ está em função do terceiro jato da imersão, sendo assim genericamente não-nulo. Logo, numa vizinhança de $\mathfrak{p}$, a condição $\operatorname{det}(\operatorname{Jac}(F)) \neq 0$ é válida. Pelo Teorema da Transversalidade de Thom (ver Apêndice A), segue que $(0,0,0)$ é valor regular de $F$ para um conjunto residual em $\mathcal{I}^{r}\left(M^{2}, \mathbb{R}^{4}\right) \subset \mathcal{C}^{r}\left(M^{2}, \mathbb{R}^{4}\right), r \geq 4$.
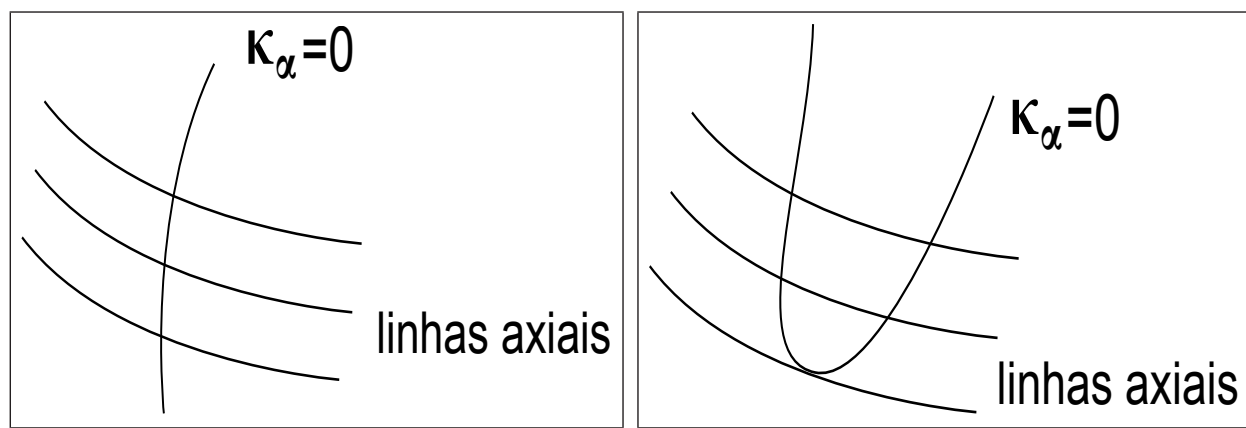

Figura 4.3: Ilustração, à esquerda, do caso em que $F^{-1}(0,0,0)=\emptyset$, e à direita e do caso $F^{-1}(0,0,0) \neq \emptyset$.

\subsection{Superfícies em torno de Ciclos Axiais Principais}

Vamos considerar nesta seção o seguinte problema: dada uma curva suave fechada $\gamma$ em $\mathbb{R}^{4}$, construir uma superfície $M^{2} \subset \mathbb{R}^{4}$ de modo que $\gamma$ seja um ciclo axial principal de $M^{2}$. Efetuamos uma parametrização local de uma superfície que contém a curva $\gamma$ (equação (4.13)) de modo a estabelecer, na Proposição 4.14, uma condição necessária e suficiente para que $\gamma$ seja reunião de linhas axiais principais.

Seja $\gamma:[0, L] \longrightarrow \mathbb{R}^{4}$ uma curva suave, simples, regular e fechada em $\mathbb{R}^{4}$, parametrizada pelo comprimento de arco $u$. Ao longo da curva $\gamma$, podemos considerar o Referencial Móvel de Frenet, denotado por $\left\{e_{1}(u), e_{2}(u), e_{3}(u), e_{4}(u)\right\}$, sendo $e_{1}(u)=\gamma^{\prime}(u)$. Tal refe- 
rencial satisfaz às equações:

$$
\left\{\begin{array}{l}
e_{1}^{\prime}(u)=\tau_{1}(u) \cdot e_{2}(u) \\
e_{2}^{\prime}(u)=-\tau_{1}(u) \cdot e_{1}(u)+\tau_{2}(u) \cdot e_{3}(u) \\
e_{3}^{\prime}(u)=-\tau_{2}(u) \cdot e_{2}(u)+\tau_{3}(u) \cdot e_{4}(u) \\
e_{4}^{\prime}(u)=-\tau_{3}(u) \cdot e_{3}(u)
\end{array}\right.
$$

Este sistema também pode ser descrito pela seguinte equação matricial:

$$
\left[\begin{array}{c}
e_{1}^{\prime}(u) \\
e_{2}^{\prime}(u) \\
e_{3}^{\prime}(u) \\
e_{4}^{\prime}(u)
\end{array}\right]=\left[\begin{array}{cccc}
0 & \tau_{1}(u) & 0 & 0 \\
-\tau_{1}(u) & 0 & \tau_{2}(u) & 0 \\
0 & -\tau_{2}(u) & 0 & \tau_{3}(u) \\
0 & 0 & -\tau_{3}(u) & 0
\end{array}\right] \cdot\left[\begin{array}{l}
e_{1}(u) \\
e_{2}(u) \\
e_{3}(u) \\
e_{4}(u)
\end{array}\right]
$$

onde, para cada $i=1,2,3, \tau_{i}:[0, L] \longrightarrow \mathbb{R}$ é função suave.

Considere a superfície parametrizada, de classe $\mathcal{C}^{r}, r \geq 5$, dada por:

$$
\begin{aligned}
\alpha(u, v) & =\gamma(u) \\
& +\left(\cos \theta(u) \cdot e_{2}(u)+\sin \theta(u) \cos \varphi(u) \cdot e_{3}(u)+\sin \theta(u) \sin \varphi(u) \cdot e_{4}(u)\right) \cdot v
\end{aligned}
$$

sendo $\theta(u)=\theta(u+L), \varphi(u)=\varphi(u+L)$.

Proposição 4.14. A curva $\gamma$ é reunião de linhas axiais principais de $\alpha$ se, e somente se,

$$
\left\{\begin{array}{l}
\theta^{\prime}(u)+\tau_{2}(u) \cdot \cos \varphi(u)=0 \\
\theta(0)=\theta_{0}
\end{array}\right.
$$

$e$

$$
\int_{0}^{L} \tau_{2}(u) \cdot \cos \beta(u) d u=2 m \pi, \quad m \in \mathbb{Z} .
$$

Para toda solução $\theta(s)$ da equação (4.14), a superfície parametrizada definida por (4.13) é regular, orientada e mergulhada em uma vizinhança da curva $\gamma$.

Demonstração: Considere a superfície parametrizada:

$$
\begin{aligned}
\alpha(u, v) & =\gamma(u) \\
& +\left(\cos \theta(u) \cdot e_{2}(u)+\sin \theta(u) \cos \varphi(u) \cdot e_{3}(u)+\sin \theta(u) \sin \varphi(u) \cdot e_{4}(u)\right) \cdot v
\end{aligned}
$$

No referencial $\left\{e_{1}(u), e_{2}(u), e_{3}(u), e_{4}(u)\right\}$, temos que:

$$
\begin{aligned}
\alpha_{u}(u, v) & =\left[1-\cos \theta(u) \tau_{1}(u) v\right] \cdot e_{1}(u) \\
& -\left[\left(\theta^{\prime}(u)+\theta_{2}(u) \cos \varphi(u)\right) \sin \theta(u)\right] v \cdot e_{2}(u) \\
& +\left[\cos \theta(u)\left(\tau_{2}(u)+\theta^{\prime}(u) \cos \varphi(u)\right)-\sin \theta(u) \sin \varphi(u)\left(\tau_{3}(u)+\varphi^{\prime}(u)\right)\right] v \cdot e_{3}(u) \\
& +\left[\sin \theta(u) \cos \varphi(u)\left(\tau_{3}(u)+\varphi^{\prime}(u)\right)+\cos \theta(u) \theta^{\prime}(u) \sin \varphi(u)\right] v \cdot e_{4}(u),
\end{aligned}
$$




$$
\alpha_{v}(u, v)=\cos \theta(u) \cdot e_{2}(u)+\sin \theta(u) \cos \varphi(u) \cdot e_{3}(u)+\sin \theta(u) \sin \varphi(u) \cdot e_{3}(u) .
$$

Para $v=0$, temos que $\alpha_{u}(u, 0)=e_{1}(u)$, e

$$
\alpha_{v}(u, v)=\cos \theta(u) \cdot e_{2}(u)+\sin \theta(u) \cos \varphi(u) \cdot e_{3}(u)+\sin \theta(u) \sin \varphi(u) \cdot e_{3}(u) .
$$

Assim $\left\{\alpha_{u}, \alpha_{v}\right\}$ é linearmente independente ao longo de $\gamma$ e portanto $\alpha$ define localmente uma superfície regular na vizinhança da curva $\gamma$.

Os coeficientes da primeira e segunda forma de $\alpha$ são:

$$
\begin{aligned}
& E(u, v)=1-2 \tau_{1}(u) \cos \theta(u) \cdot v+O_{2}(v), \\
& F(u, v)=0 \\
& G(u, v)=1
\end{aligned}
$$

De acordo com a parametrização utilizada, temos os vetores normais $N_{1}$ e $N_{2}$ dados por:

$$
N_{1}(u, 0)=\frac{1}{m_{1}(u)} \cdot\left[\sin \theta(u) \cos \varphi(u) \cdot e_{2}(u)-\cos \theta(u) \cdot e_{3}(u)\right]
$$

sendo

$$
\left(m_{1}(u)\right)^{2}=\cos ^{2} \theta(u)+\sin ^{2} \theta(u) \cos ^{2} \varphi(u) .
$$

$\mathrm{E}$

$$
\begin{aligned}
N_{2}(u, 0) & =\frac{1}{m_{2}(u)} \cdot\left[\sin \theta(u) \cos \theta(u) \sin \varphi(u) \cdot e_{2}(u)\right. \\
& +\sin ^{2} \theta(u) \sin \varphi(u) \cos \varphi(u) \cdot e_{3}(u) \\
& \left.-\left[\cos ^{2} \theta(u)+\sin ^{2} \theta(u) \cos ^{2} \varphi(u)\right] \cdot e_{4}(u)\right],
\end{aligned}
$$

com

$$
\begin{aligned}
\left(m_{2}(u)\right)^{2} & =\sin ^{2} \theta(u) \sin ^{2} \varphi(u)\left(\cos ^{2} \theta(u)+\sin ^{2} \theta(u) \cos ^{2} \theta(u)\right) \\
& +\cos ^{2} \theta(u)\left(\cos ^{2} \theta(u)+2 \sin ^{2} \theta(u) \cos ^{2} \varphi(u)\right) \\
& +\sin ^{4} \theta(u) \cos ^{4} \theta(u)
\end{aligned}
$$


Assim, os coeficientes da segunda forma fundamental são:

$$
\begin{aligned}
e_{1}(u, v) & =\frac{1}{m_{1}(u)} \cdot \tau_{1}(u) \sin \theta(u) \cos \varphi(u)+O_{1}(v), \\
f_{1}(u, v) & =\frac{1}{m_{1}(u)} \cdot\left[\left(\cos \theta(u) \theta^{\prime}(u) \cos \varphi(u)-\sin \theta(u) \sin \varphi(u) \varphi^{\prime}(u)\right) \cos \theta(u)\right. \\
& \left.-\left(\sin ^{2} \theta(u) \cos ^{2} \varphi(u)+\cos ^{2} \theta(u)\right) \tau_{2}(u)-\sin ^{2} \theta(u) \cos \varphi(u) \theta^{\prime}(u)\right]+O_{1}(v), \\
g_{1}(u, v) & =0, \\
e_{2}(u, v) & =\frac{1}{m_{2}(u)} \cdot \tau_{1}(u) \sin \theta(u) \cos \theta(u) \sin \varphi(u)+O_{1}(v), \\
f_{2}(u, v) & =-\frac{1}{m_{2}(u)} \cdot\left[\sin \theta(u)\left(\tau_{3}(u) \cos \theta(u)+\cos \varphi(u) \varphi^{\prime}(u)\right)\right. \\
& \left.-\sin \varphi(u) \cos \theta(u) \theta^{\prime}(u)\right]+O_{1}(v), \\
g_{2}(u, v) & =0 .
\end{aligned}
$$

Lembramos que os coeficientes da equação diferencial das linhas axiais (Proposição 1.4) são:

$$
\begin{aligned}
a_{0} & =4 F\left(E G-2 F^{2}\right)\left(e_{1}^{2}+e_{2}^{2}\right) \\
& -4 E\left(E G-4 F^{2}\right)\left(e_{1} f_{1}+e_{2} f_{2}\right) \\
& +4 E^{3}\left(f_{1} g_{1}+f_{2} g_{2}\right) \\
& -4 E^{2} F\left(e_{1} g_{1}+e_{2} g_{2}\right) \\
& -8 E^{2} F\left(f_{1}^{2}+f_{2}^{2}\right),
\end{aligned}
$$

e

$$
\begin{aligned}
a_{1} & =4 G\left(E G-4 F^{2}\right)\left(e_{1}^{2}+e_{2}^{2}\right) \\
& +32 E F G\left(e_{1} f_{1}+e_{2} f_{2}\right) \\
& +4 E^{3}\left(g_{1}^{2}+g_{2}^{2}\right) \\
& -8 E^{2} G\left(e_{1} g_{1}+e_{2} g_{2}\right) \\
& -16 E^{2} G\left(f_{1}^{2}+f_{2}^{2}\right) .
\end{aligned}
$$

Substituindo devidamente as expressões da primeira e segunda forma fundamental, obtemos:

$$
a_{0}(u, 0)=4 \tau_{1}(u) \sin \theta(u) \cdot\left(\tau_{2}(u) \cos \varphi(u)+\theta^{\prime}(u)\right)
$$

Como $\tau_{1}(u)>0$ e assumindo que $\theta(u)$ não é constante e identicamente nulo, segue que a curva $\gamma(u)$ será linha de curvatura axial principal se, e somente se:

$$
\theta^{\prime}(u)+\tau_{2} \cos \varphi(u)=0
$$




\section{Conclusão}

No trabalho apresentado descrevemos o comportamento das linhas axiais principais e médias na vizinhança dos pontos axiumbílicos de codimensão 1, denotados por $E_{34}^{1}$ e $E_{45}^{1}$, via o método geométrico de Lie-Cartan considerando imersões de classe $\mathcal{C}^{r}, r \geq 5$ de uma superfície $M$ em $\mathbb{R}^{4}$. Efetuando o levantamento para o fibrado projetivo da superfície, utilizamos técnicas de Teoria de Bifurcações para estudar singularidade de tipo sela-nó quadrática cujas linhas próximas se projetam na superfície obtendo as Configurações ilustradas na Figura 3.10 e Figura 3.16.

Dando sequência ao estudo de ciclos axiais principais e médios feito por Garcia e Sotomayor ( [42], [44]), descrevemos a Transformação de Primeiro Retorno, também conhecida como Transformação de Poincaré, e utilizando uma expressão local apropriada da superfície na vizinhança de um ciclo, calculamos a Fórmula Integral da Primeira Derivada e a Fórmula Integral da Segunda Derivada da Transformação de Poincaré. Com isto, efetuamos a discussão da hiperbolicidade $\left(\pi^{\prime}(0) \neq 1\right)$ e semi-hiperbolicidade $\left(\pi^{\prime}(0)=1\right.$ e $\left.\pi^{\prime \prime}(0) \neq 0\right)$ dos ciclos axiais.

De posse da expressão das fórmulas de primeira e segunda derivadas, assumimos uma hipótese geométrica genérica ao longo dos ciclos axiais e construimos perturbações no espaço das imersões de modo a demonstrar dois fatos:

- Dada uma imersão com ciclo axial não-hiperbólico, é possível encontrar imersão suficientemente próxima que torna tal ciclo hiperbólico (Teorema 4.9).

- Dada uma imersão com ciclo axial não-hiperbólico e não semi-hiperbólico, é possível encontrar imersão suficientemente próxima que torna tal ciclo semi-hiperbólico (Teorema 4.11). 


\section{Apêndice A}

\section{Teorema de Transversalidade de Thom}

Neste apêndice fazemos uma síntese dos conceitos e resultados utilizados nas seções 3.3 e 4.4. Tal conteúdo pode ser encontrado de forma mais explicativa nos capítulos 2 e 3 de [26], bem como nas referências [29] e [36].

Sejam $M$ e $N$ variedades diferenciáveis de classe $\mathcal{C}^{r}$ e denote por $\mathcal{C}^{r}(M, N)$ o conjunto das aplicações de classe $\mathcal{C}^{r}$ de $M$ em $N, 1 \leq r<\infty$. Considere $f \in \mathcal{C}^{r}(M, N)$ e $(\varphi, \mathcal{U}),(\psi, \mathcal{V})$ cartas em $M$ e $N$ respectivamente. Seja $K \subset \mathcal{U}$ um conjunto compacto tal que $f(K) \subset \mathcal{V}$, e tome $\varepsilon>0$.

Definimos uma vizinhança básica fraca

$$
\mathcal{N}^{r}(f ;(\varphi, \mathcal{U}),(\psi, \mathcal{V}), K, \varepsilon)
$$

como o conjunto das aplicações $g: M \longrightarrow N$ de classe $\mathcal{C}^{r}$ tais que $g(K) \subset \mathcal{V}$ e

$$
\left\|D^{k}\left(\psi f \varphi^{-1}\right)(x)-D^{k}\left(\psi g \varphi^{-1}\right)(x)\right\|<\varepsilon
$$

para todo $x \in \varphi(k)$ e todo $k=0, \ldots, r$.

A topologia fraca em $\mathcal{C}^{r}(M, N)$ é gerada pelos conjuntos do tipo (A.1), ou seja, a vizinhança de $f \in \mathcal{C}^{r}(M, N)$ é um conjunto contendo a interseção de um número finito de conjuntos do tipo (A.1). O conjunto $\mathcal{C}^{r}(M, N)$ munido da topologia fraca é denotado por $\mathcal{C}_{W}^{r}(M, N)$.

Vamos definir também a topologia forte, ou topologia de Whitney em $\mathcal{C}^{r}(M, N)$. Para tal, seja $\Phi=\left\{\varphi_{i}, \mathcal{U}_{i}\right\}_{i \in \Lambda}$ uma família localmente finita de cartas em $M$; isto significa que cada ponto de $M$ tem uma vizinhança a qual intersecta $\mathcal{U}_{i}$ apenas para um número finito de $i$ 's. Seja $K=\left\{K_{i}\right\}_{i \in \Lambda}$ uma família de subconjuntos compactos de $M, K_{i} \subset \mathcal{U}_{i}$, $\Psi=\left\{\psi_{i}, \mathcal{V}_{i}\right\}_{i \in \Lambda}$ uma família de cartas em $N$, e $\varepsilon=\left\{\varepsilon_{i}\right\}_{i \in \Lambda}$ uma família de números positivos. Se, para cada i, $f \in \mathcal{C}^{r}(M, N)$ é de modo que $f\left(K_{i}\right) \subset \mathcal{V}_{i}$, definimos uma vizinhança básica forte

$$
\mathcal{N}^{r}(f ; \Phi, \Psi, K, \varepsilon)
$$


como o conjunto das aplicações $g: M \longrightarrow N$ de classe $\mathcal{C}^{r}$ tais que, para cada $i \in \Lambda$, $g\left(K_{i}\right) \in \mathcal{V}_{i}$ e

$$
\left\|D^{k}\left(\psi f \varphi_{i}^{-1}\right)(x)-D^{k}\left(\psi g \varphi_{i}^{-1}\right)(x)\right\|<\varepsilon_{i}
$$

para todo $x \in \varphi_{i}\left(K_{i}\right)$ e todo $k=0, \ldots, r$.

A topologia forte em $\mathcal{C}^{r}(M, N)$ é gerada pelos conjuntos do tipo (A.2). O conjunto $\mathcal{C}^{r}(M, N)$ munido da topologia forte é denotado por $\mathcal{C}_{S}^{r}(M, N)$.

Definição A.1. Um $n$-cubo $C \subset \mathbb{R}^{n}$ de aresta $\lambda>0$ é um produto

$$
C=I_{1} \times \cdots \times I_{n}
$$

onde cada $I_{j}, j=1, \ldots, n$ é um intervalo real fechado de comprimento $\lambda$. A medida de $C$ é definida por:

$$
\mu(C)=\lambda^{n}
$$

Definição A.2 (Conjunto de Medida Nula). Dizemos que $X \subset \mathbb{R}^{n}$ é um conjunto de medida nula se, para todo $\varepsilon>0$, é possível encontrar uma família de n-cubos $C_{k}$, de modo que

$$
X \subset \bigcup C_{k}
$$

com

$$
\sum \mu\left(C_{k}\right)<\varepsilon
$$

No caso em que $X \subset M, M$ uma $n$-variedade diferenciável, dizemos que $X$ tem medida nula se, para toda carta $(\varphi, \mathcal{U})$, o conjunto $\varphi(\mathcal{U} \cap X) \subset \mathbb{R}^{n}$ satisfaz a definição de medida nula para conjuntos em $\mathbb{R}^{n}$.

De acordo com [26], pg. 68, um subconjunto fechado de medida nula em $\mathbb{R}^{n}$ (ou em uma variedade $M$ ) tem interior vazio, ou seja, é denso em parte alguma. Assim, seja $X \subset$ $M$ um conjunto com medida nula o qual é $\sigma$-compacto, ou seja, $X$ é a reunião enumerável de conjuntos compactos. Cada um desses compactos são densos em parte alguma, e portanto $M \backslash X$ é denso, pelo Teorema da Categoria de Baire ( [35], pg. 296). O conjunto complementar de $X$ é residual, ou seja, contém uma interseção enumerável de conjuntos abertos e densos. O Teorema da Categoria de Baire diz que um subconjunto residual de um espaço métrico completo é denso. Note também que a interseção enumerável de conjuntos residuais é também residual.

Teorema A.3 (Teorema de Sard). Sejam $M^{m}$ e $N^{n}$ variedades diferenciáveis e $f$ : $M \longrightarrow N$ uma aplicação de classe $\mathcal{C}^{r}$. Se

$$
r>\max \{0, m-n\}
$$


então o conjunto dos valores críticos de $f$ tem medida nula em $N$. O conjunto dos valores regulares de $f$ é residual e portanto denso.

Corolário A.4. Para todo $y_{0} \in N$, o conjunto

$$
\left\{f \in \mathcal{C}^{r}(M, N): y_{0} \text { é valor regular }\right\}
$$

é aberto e denso em $\mathcal{C}_{S}^{r}(M, N), \quad 1 \leq r \leq \infty$.

Definição A.5. Seja $f: M \longrightarrow N$ uma aplicação $\mathcal{C}^{1}, A$ uma subvariedade de $N$ e $K$ um subconjunto de $M$ :

a) Dizemos que $f$ é transversal a $A$ ao longo de $K$ (denotado por $f \pitchfork_{K} A$ ) se, para todo $x$ tal que $x \in K$ e $f(x) \in A$, temos que

$$
d f_{x}(M)+T_{f(x)} A=T_{f(x)} N
$$

b) No caso em que $K=M$, dizemos que $f$ é transversal a $A$, denotado por $f$ 币 $A$.

c) Definimos

$$
\varlimsup_{K}^{r}(M, N ; A)=\left\{f \in \mathcal{C}^{r}(M, N): f \varlimsup_{K} A\right\}
$$

$\mathrm{e}$

$$
\AA^{r}(M, N ; A)=\left\{f \in \mathcal{C}^{r}(M, N): f \AA A\right\} .
$$

Teorema A.6 (Teorema de Transversalidade, [26] pg. 74). Sejam $M$ e $N$ variedades diferenciáveis e A subvariedade de $N$. Então, para $1 \leq r \leq \infty$ :

i) $\Pi^{r}(M, N ; A)$ é residual (e portanto denso) em $\mathcal{C}^{r}(M, N)$, para ambas as topologias forte e fraca.

ii) Suponha que A seja fechado em $N$. Se $L \subset M$ é fechado (respectivamente compacto), então $\varlimsup_{L}^{r}(M, N ; A)$ é aberto e denso em $\mathcal{C}_{S}^{r}(M, N)$ (respectivamente em $\mathcal{C}_{W}^{r}(M, N)$ ).

Sejam $M, N$ variedades diferenciáveis de classe $\mathcal{C}^{r}, 0 \leq r<\infty$. Um $r$-jato de $M$ em $N$ é uma classe de equivalência $[x, f, \mathcal{U}]_{r}$ de triplas $(x, f, \mathcal{U})$, onde $\mathcal{U} \subset M$ é um conjunto aberto, $x \in \mathcal{U}$, e $f: \mathcal{U} \longrightarrow N$ é uma aplicação $\mathcal{C}^{r}$; a relação de equivalência é: $[x, f, \mathcal{U}]_{r} \sim\left[x^{\prime}, f^{\prime}, \mathcal{U}^{\prime}\right]_{r}$ se $x=x^{\prime}$ e, para algum (e portanto qualquer) par de cartas adaptadas a $f$ em $x, f$ e $f^{\prime}$ tem as mesmas derivadas até a ordem $r$. Usamos a notação:

$$
[x, f, \mathcal{U}]_{r}=j_{x}^{r} f=j^{r} f(x)
$$

para denotar o $r$-jato de $f$ em $x$. Denominamos $x$ a fonte e $f(x)$ a meta de $[x, f, \mathcal{U}]$. 
O conjunto de todos os $r$-jatos de $M$ em $N$ é denotado por $J^{r}(M, N)$. A aplicação fonte é definida por

$$
\begin{aligned}
\sigma: J^{r}(M, N) & \longrightarrow M \\
{[x, f, \mathcal{U}]_{r} } & \longmapsto x
\end{aligned}
$$

e a aplicação meta por

$$
\begin{gathered}
\tau: J^{r}(M, N) \longrightarrow N \\
{[x, f, \mathcal{U}]_{r} \longmapsto f(x)}
\end{gathered}
$$

Denote:

$$
\sigma^{-1}(x)=J_{x}^{r}(M, N), \quad \tau^{-1}(y)=J^{r}(M, N)_{y},
$$

$\mathrm{e}$

$$
J_{x}^{r}(M, N) \cap J^{r}(M, N)_{y}=J_{x, y}^{r}(M, N) .
$$

$J_{x, y}^{r}(M, N)$ é o conjunto de todos os $r$-jatos de $M$ em $N$ com fonte $x$ e meta $y$.

Considere o caso especial em que $M=\mathbb{R}^{m}$ e $N=\mathbb{R}^{n}$. Escrevemos

$$
J^{r}\left(\mathbb{R}^{m}, \mathbb{R}^{n}\right)=J^{r}(m, n)
$$

Suponha $\mathcal{U} \subset \mathbb{R}^{m}$ aberto e $f \in \mathcal{C}^{r}\left(\mathcal{U}, \mathbb{R}^{n}\right)$. O $r$-jato de $f$ em $x \in \mathcal{U}$ tem uma representação canônica, a saber, o polinômio de Taylor de $r$ de ordem $r$ em $x$. Esta aplicação polinomial de $\mathbb{R}^{m}$ em $\mathbb{R}^{n}$ é unicamente determinada pela lista das derivadas até a ordem $r$ de $f$ em $x$. Esta lista pertence ao espaço vetorial

$$
P^{r}(m, n)=\mathbb{R}^{n} \times \prod_{k=1}^{r} L_{\text {sim }}\left(\mathbb{R}^{m}, \mathbb{R}^{n}\right)
$$

onde $L_{\text {sim }}\left(\mathbb{R}^{m}, \mathbb{R}^{n}\right)$ denota o espaço vetorial das formas $k$-lineares simétricas de $\mathbb{R}^{m}$ em $\mathbb{R}^{n}$. Podemos identificar:

$$
J_{x}^{r}(m, n)=P^{r}(m, n)
$$

e

$$
J^{r}(m, n)=\mathbb{R}^{m} \times P^{r}(m, n) .
$$

Em particular, $J^{r}(m, n)$ é um espaço vetorial de dimensão finita (para $r$ finito). Se $\mathcal{U} \subset \mathbb{R}^{m}$ e $\mathcal{V} \subset \mathbb{R}^{n}$ são abertos, então $J^{r}(U, V)$ é um subconjunto aberto de $J^{r}(m, n)$.

Sejam $M^{m}$ e $N^{n}$ variedades sem bordo, com as respectivas cartas $(\varphi, \mathcal{U}),(\psi, \mathcal{V})$. Definimos a aplicação

$$
\begin{gathered}
\theta: J^{r}(U, V) \longrightarrow J^{r}(\varphi \mathcal{U}, \psi \mathcal{V}) \\
j_{x}^{r} f \longmapsto j_{f(x)}^{r}\left(\psi f \varphi^{-1}\right)
\end{gathered}
$$

a qual é uma bijeção que associa cada jato ao jato de sua representação local. $J^{r}(\varphi \mathcal{U}, \psi \mathcal{V})$ é um conjunto aberto no espaço vetorial $J^{r}(m, n)$, o qual é isomorfo ao espaço euclideano. Portanto, $\left(\theta, J^{r}(\mathcal{U}, \mathcal{V})\right.$ é uma carta em $J^{r}(M, N)$. No caso em que $M$ e $N$ são variedades de classe $\mathcal{C}^{s}, 1 \leq r<s \leq \infty$, temos que $J^{r}(M, N)$ é uma variedade de classe $\mathcal{C}^{s-r}$. 
Definição A.7. Sejam $M$ e $N$ variedades de classe $\mathcal{C}^{s}, 1 \leq r<s \leq \infty$, e $f \in \mathcal{C}^{s}(M, N)$.

a) Definimos r-prolongamento de $f$ por

$$
\begin{aligned}
j^{r} f: M & \longrightarrow J^{r}(M, N) \\
x & \longmapsto j^{r} f(x)
\end{aligned}
$$

b) Definimos a aplicação jato por

$$
\begin{aligned}
j^{r}: \mathcal{C}^{s}(M, N) & \longrightarrow \mathcal{C}^{s-r}\left(M, J^{r}(M, N)\right) \\
f & \longmapsto j^{r} f
\end{aligned}
$$

c) Se $A$ é uma subvariedade de $J^{r}(M, N)$, definimos

$$
\pi^{s}\left(M, N ; j^{r}, A\right)=\left\{h \in \mathcal{C}^{s}(N, M): j^{r} h \text { } A\right\} .
$$

Teorema A.8 (Teorema de Transversalidade de Jatos, [26] pg. 80). Sejam M, Nvariedades suaves sem bordo, e seja $A \subset J^{r}(M, N)$ uma subvariedade suave. Suponha que $1 \leq r<s \leq \infty$. Então $\AA^{s}\left(M, N ; j^{r}, A\right)$ é residual, e portanto denso, em $\mathcal{C}_{S}^{s}(M, N)$. Caso A seja fechado, $\AA^{s}\left(M, N ; j^{r}, A\right)$ é também aberto em $\mathcal{C}_{S}^{s}(M, N)$. 


\section{Referências Bibliográficas}

[1] Andronov, A., Gordon, I., Leontovich, E., Maier, G. Theory of Bifurcations of Dynamical Systems on a Plane. Israel Program for Scientific Translations, John Wiley, New York, 1973.

[2] Asperti, A. C., Immersions of Surfaces into 4-Dimensional Spaces with Nonzero Normal Curvature. Ann. di Mat. Pura et App. IV, vol. CXXV, 313-329, 1980.

[3] Asperti, A. C., Some Generic Properties of Riemannian Immersions. Bol. Soc. Bras. Mat., vol. 11, n. 2, 191-216. 1980.

[4] Barreto, R. F., Bifurcações de Linhas de Curvatura em torno de Conexões de Pontos Umbílicos. Tese de Doutorado. IME/USP. 2000.

[5] Bittes, K. F., Ciclos Principais, Médios e Assintóticos e Bifurcações. Dissertação de Mestrado. IME/UFG. 2003.

[6] Boothby, W. M., An Introduction to Differentiable Manifolds and Riemmanian Geometry, Revised Second Edition, San Diego: Academic Press, 2003.

[7] Burnside, W., Panton, A. The theory of Equations. Dover Publications, Inc. New York. 1912.

[8] Camacho, C., Lins Neto, A. Teoria Geométrica das Folheações, Rio de Janeiro: IMPA, 1979.

[9] Chow, S-N., Li, C., Wang, D., Normal Forms and Bifurcation of Planar Vector Fields, Cambridge University Press, 1994.

[10] doCarmo, M. P., Geometria Diferencial de Curvas e Superfícies. Rio de Janeiro, SBM. 2005.

[11] doCarmo, M. P., Geometria Riemanniana, Terceira Edição. Rio de Janeiro, IMPA. 2005 .

[12] Dumortier, F., Llibre, J., Artés, J., Qualitative Theory of Planar Differential Systems. Springer-Verlag Berlin Heidelberg, 2006. 
[13] Ganchev, G., Milousheva, V., Invariants and Bonnet-Type Theorem for Surfaces in $\mathbb{R}^{4}$. Central European Journal of Mathematics, Vol. 8, Issue 6, pp 993-1008, Dec. 2010.

[14] Garcia, R., Linhas de Curvatura de Hipersuperfícies Imersas no Espaço $\mathbb{R}^{4}$. Tese de Doutoramento, IMPA, 1989.

[15] Garcia, R., Mello, L. F., Sotomayor, J., Principal Mean Curvature Foliations on Surfaces Immersed in $\mathbb{R}^{4}$. EQUADIFF 2003, 939-950, World Sci. Publ., Hackensack, NJ, 2005.

[16] Garcia, R., Sotomayor, J., Lines of Mean Curvature on Surfaces Immersed in $\mathbb{R}^{3}$. Qual. Theory Dyn. Syst. 4, n. 2, 263-309, 2004.

[17] Garcia, R., Sotomayor, J., Structurally Stable Configurations of Lines of Mean Curvature and Umbilic Points on Surfaces Immersed in $\mathbb{R}^{3}$. Publ. Mat. 42, 431-466. 2001.

[18] Gutierrez, C., Guíñez, V., Simple Umbilic Points on Surfaces Immersed in $\mathbb{R}^{4}$. Discr. and Cont. Dyn. Systems, vol. 9, n. 4, julho 2003.

[19] Gutierrez, C., Guíñez, V., Quartic Differential Forms Associated to Couples of Transversal Nets with Singularities. Matemática Contemporânea, vol. 31, 29-52. 2006.

[20] Gutierrez, C., Guíñez, V., Castañeda, A., Quartic Differential Forms and Transversal Nets with Singularities. Discr. and Cont. Dyn. Systems, vol. 26, n. 1, janeiro 2010.

[21] Gutierrez, C., Guadalupe, I., Tribuzy, R., Guíñez, V., Lines of Curvature on Surfaces immersed in $\mathbb{R}^{4}$. Bol. Soc. Bras. Mat., vol. 28, n. 2, 233-251. 1997.

[22] Gutierrez, C., Garcia, R., Sotomayor, J., Bifurcations of Umbilic Points and Related Principal Cycles. Journ. Dyn. and Diff. Eq., vol. 16, 321-346. 2004.

[23] Gutierrez, C., Sotomayor, J., Lines of Curvature and Umbilic Points on Surfaces. Brazilian 18 ${ }^{\text {th }}$ Math. Coll., IMPA, 1991, reprinted as Structurally Configurations of Lines of Curvature and Umbilic Points on Surfaces, Monografias del IMCA, Lima, Peru, 1998.

[24] Gutierrez, C., Sotomayor, J., Structurally Stable Configurations of Lines of Principal Curvature. Asterisque, 98-99: 195-215, 1982.

[25] Gutierrez, C., Sotomayor, J., Closed Principal Lines and Bifurcation. Boletim Soc. Bras. Mat., vol 17. n. 1, 1-19, 1986 
[26] Hirsch, M., Differential Topology. Graduate Texts in Mathematics; 33. SpringerVerlag, New York, 1976.

[27] Janson, S., Resultant and Discriminant of Polynomials. Uppsala University. Sweden. in: http://www2.math.uu.se/ svante/papers/index.html

[28] Kuznetsov, Y., Elements of Applied Bifurcation Theory, Third Edition. Applied Mathematical Sciences, 112. Springer-Verlag, New York, 2004.

[29] Levine, H. T., Singularities of Differentiable Mappings, Lect. Notes in Math. 192, 1971.

[30] Little, J. A., On Singularities of Submanifolds of Higher Dimensional Euclidean Spaces. Ann. Mat. Pura Appl., 83: 261-335, 1969.

[31] Mello, L.F., Linhas de Curvatura Direcional Média em Superfícies Imersas em $\mathbb{R}^{4}$. Tese de Doutorado. IME/USP. 2001.

[32] Mello, L. F., Mean Directionally Curved Lines on Surfaces Immersed in $\mathbb{R}^{4}$. Publ. Mat., 47, 415-440, 2003.

[33] Mello, L. F., Line Fields on Surfaces Immersed in $\mathbb{R}^{4}$. EQUADIFF 2003, 951-956, World Sci. Publ., Hackensack, NJ, 2005.

[34] Montesinos, A., Softwares disponíveis na página pessoal: Universitat de Valencia, www.uv.es/montesin/. 2009.

[35] Munkres, J. Topology, 2. ed. Prentice Hall, Inc. 2000.

[36] Loibel, G. F., Singularidades de Aplicações Diferenciáveis. Sexto Colóquio Brasileiro de Matemática, Poços de Caldas, 1967.

[37] Palais, R. S., Terng, C-L., Critical Point Theory and Submanifold Geometry. Lectures Notes in Mathematics 1353: Springer Verlag, 1988.

[38] Roussarie, R., Bifurcations of Planar Vector Fields and Hilbert's Sixteenth Problem. Progress in Mathematics, vol. 164. Birkhäuser Verlag, 1998.

[39] Sotomayor, J., Curvas Definidas por Equações Diferenciais no Plano. Brazilian $13^{\text {th }}$ Math. Coll., IMPA, 1981.

[40] Sotomayor, J., Lições de Equações Diferenciais Ordinárias. Projeto Euclides, CNPq, IMPA, 1979.

[41] Sotomayor, J., Singularidades de Aplicações Diferenciáveis. Rio de Janeiro: IMPA, 1976. 
[42] Sotomayor, J., Garcia, R., Differential Equations of Classical Geometry, a Qualitative Theory. Publicações Matemáticas. IMPA. 2009.

[43] Sotomayor, J., Garcia, R., Codimension Two Umbilic Points on Surfaces Immersed in $\mathbb{R}^{3}$. Discr. Cont. Dyn. Syst., 17, n.02, 293-308, 2007.

[44] Sotomayor, J., Garcia, R., Lines of Axial Curvature on Surfaces Immersed in $\mathbb{R}^{4}$. Diff. Geom. and its Applications. 12: 253-269, 2000.

[45] Sotomayor, J., Garcia, R., Lines of Curvature on Surfaces, Historical Comments and Recent Developments. SP Journal of Math Sciences, 2: 99-143, 2008.

[46] Sotomayor, J., Garcia, R., Lines of Curvature near Principal Cycles. Annals of Global Analysis and Geometry, 10: 275-289, 1992.

[47] Sotomayor, J., Gutierrez, C., Periodic Lines of Curvature Bifurcating from Darbouxian Umbilical Connections. Springer Lect. Notes on Math., (1455), 1991.

[48] Spindola, F., Sotomayor, J., Garcia, R., Axiumbilic Singular Points on Surfaces Immersed in $\mathbb{R}^{4}$ and their Generic Bifurcations. Journal of Singularities, 124-146, vol. 10 (2014).

[49] Spindola, F., Grupos de Lie, Ações Próprias e a Conjectura de Palais-Terng. Dissertação de Mestrado em Matemática, orientada por M. M. Alexandrino. Universidade de São Paulo. (2008).

[50] Struik, D., Lectures on Classical Differential Geometry. Addison-Wesley, reprinted by Dover, 1988 .

[51] Wong, Y-C., A New Curvature Theory for Surfaces in Euclidean 4-Spaces. Comm. Math. Helv., 26, 152-170, 1952. 